\title{
Ranking Sets of Objects: The Complexity of Avoiding Impossibility Results
}

Jan Maly

Institute for Logic and Computation, TU Wien, Vienna, Austria
JMALY@DBAI.TUWIEN.AC.AT

\begin{abstract}
The problem of lifting a preference order on a set of objects to a preference order on a family of subsets of this set is a fundamental problem with a wide variety of applications in AI. The process is often guided by axioms postulating properties the lifted order should have. Well-known impossibility results by Kannai and Peleg and by Barberà and Pattanaik tell us that some desirable axioms - namely dominance and (strict) independence - are not jointly satisfiable for any linear order on the objects if all non-empty sets of objects are to be ordered. On the other hand, if not all non-empty sets of objects are to be ordered, the axioms are jointly satisfiable for all linear orders on the objects for some families of sets. Such families are very important for applications as they allow for the use of lifted orders, for example, in combinatorial voting. In this paper, we determine the computational complexity of recognizing such families. We show that it is $\Pi_{2}^{p}$-complete to decide for a given family of subsets whether dominance and independence or dominance and strict independence are jointly satisfiable for all linear orders on the objects if the lifted order needs to be total. Furthermore, we show that the problem remains coNP-complete if the lifted order can be incomplete. Additionally, we show that the complexity of these problems can increase exponentially if the family of sets is not given explicitly but via a succinct domain restriction. Finally, we show that it is NP-complete to decide for a family of subsets whether dominance and independence or dominance and strict independence are jointly satisfiable for at least one linear order on the objects.
\end{abstract}

\section{Introduction}

Modeling preferences over alternatives is a major challenge in many areas of AI, for example in knowledge representation and, especially, in computational social choice. If the number of alternatives is small enough, preferences are most often modeled as a total order. However, in many applications the alternatives are 'combinatorial', for example bundles of objects in packing or allocation problems (Bouveret, Chevaleyre, \& Maudet, 2016), or committees in voting (Lang \& Xia, 2016; Faliszewski, Skowron, Slinko, \& Talmon, 2017). In such situations, the number of alternatives grows exponentially with the number of objects which makes it unfeasible for agents to specify a full preference relation over all alternatives.

Different approaches to solving this problem have been discussed in the literature. One approach - which we will consider in this paper - is inferring an order on sets of alternatives from an order on the alternatives. This is also called lifting an order from objects to sets of objects. This approach occurs frequently in different applications in computer science, for example in the fair allocation of indivisible goods (Bouveret et al., 2016), in the study of strategic behaviors in voting with a tie-breaking mechanism (Fishburn, 1972; Barberà, 1977; Brandt, Saile, \& Stricker, 2018), in decision making when there is uncertainty about the consequences of an action (Larbi, Konieczny, \& Marquis, 2010) or in structured 

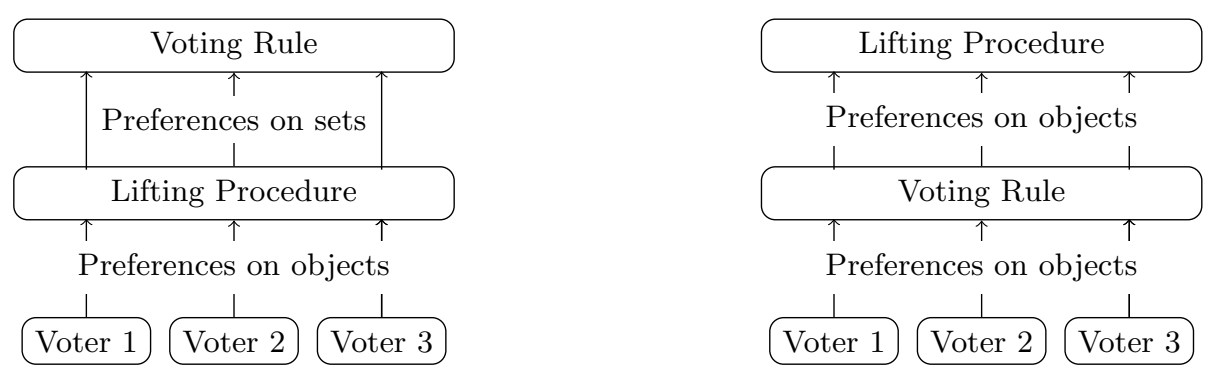

Figure 1: The use of lifted orders in voting

argumentation, if we want to compare arguments according to the strengthen of the (defeasible) assumptions they are based on (Beirlaen, Heyninck, Pardo, \& Straßer, 2018; Maly \& Wallner, 2021).

One particularly interesting setting in which sets of objects need to be ranked is combinatorial voting (Lang \& Xia, 2016), where many approaches for generating a ranking on sets of objects have been studied, none of which can be considered completely convincing if the preferences are not seperable (see Related Work). Here, the order lifting approach could offer a new and promising approach to dealing with the intractable number of alternatives. Indeed, a single winner (ordinal) voting rule combined with an order lifting procedure would immediately yield a combinatorial voting method, either by applying the lifting procedure to the input of the voting rule or to its output (see Figure 1).

However it is not clear how such a lifted order on sets can be defined. Barberà, Bossert, and Pattanaik (2004) give an excellent survey on the progress that has been made to solve this question. Essentially, there are two ways to study the order lifting approach. First, one can analyze specific methods to infer an order on sets from an order on the objects (see e.g. Moretti \& Tsoukiàs, 2012). Second, one can analyze which properties an optimal order on the sets should have in a given setting. These desirable properties can then be formulated as axioms and one can try to identify orders that satisfy these axioms. In some settings, it is possible to find orders that satisfy all properties deemed desirable in the setting (see e.g. Pattanaik \& Xu, 1990). Unfortunately, in many other settings no order can satisfy all desirable properties at once. One of the most important of these so-called impossibility results states that two axioms called Dominance and Independence are in general incompatible (Kannai \& Peleg, 1984). Dominance states, intuitively, that removing the least preferred element from a set improves the set and removing the most preferred element worsens the set. Independence states, roughly, that if a set is preferred to another set and the same element is added to both sets, this preference cannot be reversed.

Kannai and Peleg (1984) showed that dominance and independence are incompatible if one wants to order all subsets of a set with at least six elements. In the same year Barberà and Pattanaik (1984) showed that dominance and a strengthening of independence called strict independence are incompatible already if one wants to order all subsets of a three element set. These results assume that all subsets of a given set need to be ordered, but in many applications only some of these subsets are possible alternatives. For example, it is very common in multiwinner or combinatorial voting to have some form of domain restrictions (Lang \& Xia, 2016; Kilgour, 2016). Now, it is possible to construct arbitrary large families of sets - for example families of disjoint sets - that can be ordered with an 
order satisfying dominance and (strict) independence. ${ }^{1}$ Motivated by this observation, Maly and Woltran (2017) have shown that it is NP-complete to decide whether a given order on elements can be lifted to an order satisfying dominance and (strict) independence.

However, for applications in voting or other social choice problems, it is necessary to fix a voting method before the ballots are collected. Therefore, it is more important to know for a given family of sets if dominance and (strict) independence are compatible for any preference order the agents may report. ${ }^{2}$ Following Maly, Truszczynski, and Woltran (2019) we call families of sets for which any possible order on the elements can be lifted to an order satisfying dominance and (strict) independence strongly orderable with respect to dominance and (strict) independence. Maly et al. (2019) studied this concept for a specific class of families of sets, namely for families of sets that can be represented as the family of all sets of vertices that induce a connected subgraphs in a given graph. One of their main results is a classification result that implies that strong orderability with respect to dominance and strict independence can be decided in polynomial time for families in this restricted class.

In this paper, we show that this result cannot be generalized to arbitrary families of sets. We show that it is in general $\Pi_{2}^{p}$-complete to decide whether a family of sets is strongly orderable with respect to dominance and strict independence. This result also holds if we replace strict independence by independence or additionally require a natural axiom called the extension rule. These results assume that we require the order on the family of sets to be total. However, some authors argue that it is more sensible to only require incomplete preferences in combinatorial domains (Boutilier \& Rosenschein, 2016). Voting rules that facilitate the aggregation of partial orders or even weaker preference models exist (Xia \& Conitzer, 2011; Terzopoulou \& Endriss, 2019). Therefore, we investigate how much the complexity of the studied problems can be reduced by dropping the requirement that the lifted order needs to be total. In particular, we show that it is coNP-complete to decide whether a family of sets is strongly orderable if we require the order on the family to be a partial order.

These results assume that the family of sets is given explicitly. However, in many applications, the family of sets is only given implicitly due to its size which necessitates the use of lifted orders in the first place. For example, the domain in combinatorial voting is often given as a condition that has to be satisfied by the admissible sets. Such conditions can for example be formulated as propositional formulas (Lang \& Xia, 2016). These formulas are normally exponentially smaller than the actual family of sets, which can increase the complexity of deciding if the family is strongly orderable. On the other hand, families of sets must have some internal structure to be succinctly represented. This internal structure may decrease the complexity of the problem, as is the case for the domain restrictions considered by Maly et al. (2019). We show - for boolean circuits, a succinct representation that is well studied in the literature - that succinct representation can lead to a massive blow up in complexity. It turns out that it can be NEXP-hard to decide whether a succinctly represented family is strongly orderable with respect to dominance and strict independence

1. We write '(strict) independence' as a shorthand for 'independence or strict independence'.

2. Observe that the hardness of this problem does not follow from the hardness of the aforementioned problem treated by Maly and Woltran (2017). 
for total orders and coNEXP-complete for partial orders. The first result also holds if only independence is required instead of strict independence.

Finally, in some applications, for example when determining most preferred models in a knowledge representation formalism (Brewka, Niemelä, \& Truszczyński, 2003), we may want to use lifted rankings when possible and only require more information from the users if they submitted a ranking that cannot be lifted. In this case, it is valuable to know if there is no chance that a submitted ranking can be lifted such that we can ask for more information immediately. Therefore, we also study the complexity of deciding whether a family of sets is weakly orderable, that is if there is at least one order on the elements that can be lifted to an order satisfying dominance and (strict) independence. In particular, we show that it is NP-complete to decide whether a family of sets is weakly orderable with respect to dominance and strict independence. This result also holds if the extension rule is additionally required and if only a partial order is required.

Together, our results give a nearly complete picture of the complexity of determining whether dominance and (strict) independence are compatible on a given family of sets. To conclude the paper, we investigate the robustness of the obtained hardness results to modifications of the dominance axiom. For example, one could define a variation of dominance where several elements can be added at once. We show that deciding whether strict independence is jointly satisfiable with any dominance-like axiom is NP-complete.

\subsection{Related Work}

The order lifting problem has been extensively studied by authors from a wide range of scientific disciplines, including economists, mathematicians, and computer scientists. It is beyond the scope of this paper to give a complete overview over this literature. Instead, we give only a very short overview over the history of the order lifting problem.

The idea of ranking sets of objects based on a ranking of the objects is very old. For example, since antiquity humans order words lexicographically based on an order on the letters of the alphabet. Daly (1967) gives an interesting account of the development of this technique by ancient scholars. Other orders, like ordering by maximal elements are most probably as old. A greater academic interest in the order lifting problem was sparked by the famous result by Gibbard (1973) and Satterthwaite (1975) that no resolute voting rule can be strategyproof. Studying the strategyproofness of irresolute voting rules necessitated the definition of rankings of sets of candidates based on rankings of candidates. Noteworthy attempts were made by Fishburn (1972), Gärdenfors (1976) and Kelly (1977). Additionally, lottery based rankings were often considered (Duggan \& Schwartz, 2000). These are still the most widely used rankings for studying the strategyproofness of irresolute voting rules (Brandt, Brill, \& Harrenstein, 2016; Barberà, 2011).

The axiomatic approach to the order lifting problem considered in this paper is significantly younger. First works were published from the fifties onward, for example by Kraft, Pratt, and Seidenberg (1959) and Kim and Roush (1980). The seminal result that sparked a lot of interest in this area was Kannai and Peleg's famous impossibility result (Kannai \& Peleg, 1984). We will discuss this result, that is one of the key motivations of this paper, in Section 2.3 in detail. In the following years, a significant number of papers more or less directly inspired by Kannai and Peleg's result were published. Important examples include 
papers by Barberà and Pattanaik (1984), Holzman (1984), Barberà, Barret, and Pattanaik (1984), Fishburn (1984), Bandyopadhyay (1988), Bossert (1995), Kranich (1996), Bossert, Pattanaik, and Xu (2000) and Dutta and Sen (2005). For a complete overview over this line of research, we refer the reader again to the great survey by Barberà et al. (2004).

More recent developments include characterization results for decision making under complete uncertainty (Larbi et al., 2010; Bossert \& Suzumura, 2012) as well as novel approaches for ranking sets of interacting elements (Moretti \& Tsoukiàs, 2012; Lucchetti, Moretti, \& Patrone, 2015). Furthermore, Geist and Endriss (2011) managed to show several new impossibility results with computer generated proofs using SAT-solving methods. Finally, the social ranking problem of ranking elements based on a ranking of subsets, which could be seen as the dual of the order lifting problem, has received a lot of attention lately. The problem was first introduced by Moretti and Öztürk (2017) and considerable progress has been made since then (Haret, Khani, Oztürk, \& Meltem, 2018; Khani, Moretti, \& Oztürk, 2019; Bernardi, Lucchetti, \& Moretti, 2019; Allouche, Escoffier, Moretti, \& Öztürk, 2020). However, to our knowledge, all of the previous works on the axiomatic approach to the order lifting problem required a ranking of all possible subsets, with the notable exception of Bossert (1995), who studied rankings of subsets with fixed cardinality, and Maly and Woltran (2017) as well as Maly et al. (2019) on whose work we explicitly build. In this sense, the work presented here is clearly different from the existing literature on the order lifting problem.

More generally, the work presented in this paper can also be seen as a contribution to an area of research in AI concerned with models of preferences for combinatorial domains. One successful approach to dealing with combinatorial domains that has been extensively studied is the use of concise implicit preference models (Domshlak, Hüllermeier, Kaci, \& Prade, 2011; Kaci, 2011). Our research can be seen as belonging to this line of research. Whenever there is a (set of) lifted orders satisfying a collection of axioms, then the original order on elements of a set can serve as a concise representation of the lifted order on the much larger domain of subsets of that set. In general, for any succinct representation, it is crucial to know the complexity of reasoning about lifted orders based on the "ground" information about preferences on elements. In this sense, our research can be seen as a first step in studying of the use of lifted orders as a tool for implicit representations. In contrast to the lifted order approach, most implicit preference models in the literature either build on logical languages (Dubois \& Prade, 1991; Brewka, Benferhat, \& Le Berre, 2004; Brewka et al., 2003) or employ intuitive graphical representations such as lexicographic trees (Booth, Chevaleyre, Lang, Mengin, \& Sombattheera, 2010; Bräuning \& Hüllermeier, 2012; Liu \& Truszczynski, 2015), CP-nets (Boutilier, Brafman, Domshlak, Hoos, \& Poole, 2004) or CI-nets (Bouveret, Endriss, \& Lang, 2009). To this date, it is unclear whether the rankings obtained by such formalisms satisfy desirable properties that are formalized in the axiomatic approach.

Finally, many other approaches to combinatorial voting have been considered in the literature. The easiest solution is often to vote on each candidate separately. However, this approach only works well if the voters have separable preferences, i.e., if the preference on having a candidate in the committee is independent on who else is in the committee. Another option is eliciting the top ranked committee. Then one can, for example, infer a preference order on the committees via a distance measure like the Hamming distance. This 
approach minimizes the communication cost but only takes very little of the agent's full preferences into account. Alternatively, one can ask the agents to specify their preferences using a CP-net or similar representations. This can be very effective but requires the agents to learn a non-trivial preference representation. In many cases this is an unacceptable requirement. Finally, there are some voting rules that select a winning committee directly from a preferences over candidates. This approach has been mainly explored for committees of fixed size. For more information on all of these approaches, we refer the reader to the excellent survey by Lang and Xia (2016).

\subsection{Organization of the Paper}

In the next section, we will introduce the necessary technical background for our results. First, we discuss some general preliminaries (Subsection 2.1). Then, we introduce the four axioms that are the main focus of the paper (Subsection 2.2) and discuss two famous impossibility results regarding these axioms (Subsection 2.3). We define the three possible degrees to which a family can be considered orderable (Subsection 2.4). To conclude the section, we introduce two variants of our main axioms and their relation to the original axioms (Subsection 2.5). These will be useful to prove our complexity results. In Section 3, we discuss our complexity results, focusing first on strong orderability with respect to total orders (Subsection 3.1) and partial orders (Subsection 3.2). Then, we discuss the effect of succinct representation on our complexity results in Subsection 3.3. Subsequently, we consider weak orderability (Subsection 3.4) and close the section by studying the effect of strengthening dominance on our complexity results (Subsection 3.5). Finally, in Section 4 we summarize and discuss our results.

\section{Background}

In the following, we will introduce the order lifting problem, the axioms that we consider as well as the famous impossibility results regarding these axioms.

\subsection{General Preliminaries}

All sets we consider in the paper are finite. A binary relation is called a preorder if it is reflexive and transitive and is called a weak order if it is reflexive, transitive and total. ${ }^{3} \mathrm{~A}$ preorder is called a partial order if it is also antisymmetric and a weak order is called linear if it is also antisymmetric. If $\preceq$ is a weak order on a set $X$, the corresponding strict order $\prec$ on $X$ is defined by $x \prec y$ if $x \preceq y$ and $y \npreceq x$, where $x, y$ are arbitrary elements of $X$; the corresponding equivalence or indifference relation $\sim$ is defined by $x \sim y$ if $x \preceq y$ and $y \preceq x$. If $\preceq$ is linear then $x \sim y$ holds only if $x=y$. We call the linear order $1<2<3<\ldots$ the natural linear order on the natural numbers. If objects are identified with the natural numbers then we also call this order the natural order on these objects.

For a linear order $\preceq$ on a set $A$, we write $\max _{\preceq}(A)$ for the maximal element of $A$ with respect to $\preceq$. Similarly, we write $\min \preceq(A)$ for the minimal element of $A$ with respect to $\preceq$. If no ambiguity arises, we drop the reference to the relation from the notation.

3. Weak orders are also called total preorders or just orders. 
Given a set $X$ and a linear order $\leq$ on $X$, the order lifting problem consists of deriving from $\leq$ an order $\preceq$ on a family $\mathcal{X} \subseteq \mathcal{P}(X) \backslash\{\emptyset\}$ of non-empty subsets of $X$, guided by axioms formalizing some natural desiderata for such lifted orders. Generally, these axioms take $\leq$ into account. Therefore, an order $\preceq$ on $\mathcal{X}$ in general only satisfies an axiom with respect to a specific linear order $\leq$ on $X$.

\section{The Order Lifting Problem for a set of axioms $\mathcal{A}$}

Input: A set $X$, a linear order $\leq$ on $X$ and a family $\mathcal{X} \subseteq \mathcal{P}(X) \backslash\{\emptyset\}$.

Goal: $\quad$ Find an order $\preceq$ on $\mathcal{X}$ that satisfies all axioms in $\mathcal{A}$ with respect to $\leq$.

In most applications, the set of objects either represents a collection of objects that the agent receives as a whole (e.g. fair division) or a set of possible outcomes from which one will be selected (e.g. in voting with tie-breaking). The first case is often called the conjunctive interpretation and the second case the disjunctive interpretation. Moreover, observe that we did not specify what kind of order $\preceq$ should be. In the following, we will consider the problem of lifting to a variety of different types of orders. The most common case will be lifting to a weak order, which is also the problem considered most often in the literature. However, we will also consider lifting to preorders, partial orders and linear orders. Furthermore, we note that we assume that $\mathcal{X}$ only contains nonempty sets because one of our axioms, namely dominance, immediately leads to a contradiction if the empty set is contained in $\mathcal{X}$.

For the uniformity of notation, we will stick to the following conventions: In any instance of the order lifting problem, we will use uppercase letters to denote the set of objects or ground set, for example, $X$ or $Y$, and lowercase letters or natural numbers to denote its elements. We use calligraphic letters for the family of subsets, for example, $\mathcal{X}$ or $\mathcal{Y}$ and uppercase letters at the beginning of the alphabet for its elements, i.e., for subsets of the ground set. Similarly, we use $\leq$ for the linear order on the ground set, possible with an index for uniqueness, and the calligraphic $\preceq$ for any order on the family of subsets, also possibly with an index.

\subsection{The Main Axioms}

In this section we introduce the four axioms that will be the main focus of our investigation, namely dominance, independence, strict independence and the extension rule. The definitions of these axioms are not entirely consistent in the literature. We will essentially follow Barberà et al. (2004) with our definitions. However, we need to a add a condition that states that the axiom is only applicable if a set is in the family of sets that we want to lift to. As Barberà et al. (2004) only consider the case $\mathcal{X}=\mathcal{P}(X) \backslash\{\emptyset\}$, such a condition is not needed in their version of the axioms. ${ }^{4}$

Throughout this section, we will demonstrate the effect of the axioms on the following toy example: Let $S_{\text {oy }}=\{1,2,3,4\}$ and let $\leq$ be the natural linear order on $S_{o y}$. Furthermore,

4. Observe that another way of adapting the axioms to the setting $\mathcal{X} \neq \mathcal{P}(X) \backslash\{\emptyset\}$ would be, to demand that the lifted order $\preceq$ is a binary relation on $\mathcal{P}(X) \backslash\{\emptyset\}$ that satisfies all axioms and the restriction of $\preceq$ to $\mathcal{X}$ is a weak order. A study of this more restrictive approach is left for future work. 
let

$$
\mathcal{T}_{\text {oy }}=\{\{2\},\{4\},\{2,4\},\{3,4\},\{1,2,4\},\{1,4\}\} .
$$

Now, let us introduce our main axioms. We begin with the so-called dominance axiom.

\section{Dominance}

For all $A \in \mathcal{X}$ and all $x \in X$, such that $A \cup\{x\} \in \mathcal{X}$ :

$$
\begin{aligned}
& y<x \text { for all } y \in A \text { implies } A \prec A \cup\{x\} ; \\
& x<y \text { for all } y \in A \text { implies } A \cup\{x\} \prec A .
\end{aligned}
$$

Any relation $\preceq$ on $\mathcal{T}_{\text {oy }}$ that satisfies dominance with respect to $\leq$ must set $\{2\} \prec\{2,4\}$, $\{2,4\} \prec\{4\},\{3,4\} \prec\{4\},\{1,2,4\} \prec\{2,4\}$ and $\{1,4\} \prec\{4\}$.

Dominance is often also called Gärdenfors' principle after Peter Gärdenfors who introduced a version of the axiom (Gärdenfors, 1976). It states that adding an element to a set that is better than all elements already in the set increases the quality of the set and, similarly, adding a element worse than all elements in the set decreases the quality of the set.

Dominance is often desirable if the order $\preceq$ should reflect the average quality of sets. If sets represent possible outcomes, they can often be ranked by expected utility, which equals the average quality of the elements if elements are sampled with uniform probability. Such a ranking naturally satisfies dominance. Therefore, dominance is often desirable under the disjunctive interpretation of sets, where the sets represent incompatible alternatives from which one is chosen randomly. Consider, for example, an election where a voter knows that depending on his vote different sets of candidates will be tied for the first place. Furthermore, he knows that the final winner will be chosen from the tied candidates randomly. In such situations, dominance is a natural desideratum (Can, Erdamar, \& Sanver, 2009).

Observe that there are different formulations of dominance in the literature. The two most important ones are the definition given here and a version where several elements can be added at the same time (see Definition 50 for a formal statement of this version) that could be called set-dominance. Dominance and set-dominance, are equivalent if $\mathcal{X}=$ $\mathcal{P}(X) \backslash\{\emptyset\}$ and hence are used interchangeably in the literature. However, if $\mathcal{X} \neq \mathcal{P}(X) \backslash\{\emptyset\}$, then the two version constitute different axioms, as do many other possible formulations of the axiom. Given that dominance and independence are already hard to jointly satisfy, we will focus on the weakest possible formulation of the axioms for the main part of the paper. However, other definitions also hold intuitive appeal and there is not necessarily a single best formulation of dominance if $\mathcal{X} \neq \mathcal{P}(X) \backslash\{\emptyset\}$. Therefore, we will studied the effect of using other, stronger formulations of dominance in Section 3.5. Fortunately, our results indicate that changing the formulation of dominance does not seem to influence the complexity of the studied problems.

Several commonly used orders on families of sets satisfy dominance. For example, the following maxmin-based ${ }^{5}$ order satisfies dominance on all families of sets.

5. Formally, we call an order $\preceq$ a maxmin-based order if there exists an order $\preceq^{*}$ on $X \times X$ such that $A \preceq B$ holds if and only if $(\min (A), \max (A)) \preceq^{*}(\min (B), \max (B))$ holds (Barberà et al., 2004, p.13). 
Example 1. Let $X$ be a set, $\leq$ a linear order on $X$ and $\mathcal{X} \subseteq \mathcal{P}(X) \backslash\{\emptyset\}$. Then, we can define a weak order $\preceq_{m m}$ on $\mathcal{X}$ by $A \preceq_{m m} B$ for $A, B \in \mathcal{X}$ if

- $\max (A)<\max (B)$ or

- $\max (A)=\max (B)$ and $\min (A) \leq \min (B)$.

Observe that this is the maxmin-based order defined by the lexicographic order on $X \times X$. It is straightforward to check that this is a weak order. Furthermore, $\preceq_{m m}$ satisfies dominance: Let $x \in X$ and $A, A \cup\{x\} \in \mathcal{X}$. Assume $\max (A)<x$. Then, $\max (A)<\max (A \cup\{x\})=x$ and hence $A \prec_{m m} A \cup\{x\}$. On the other hand, if $x<\min (A)$, then $\max (A \cup\{x\})=\max (A)$ and $\min (A \cup\{x\})=x<\min (A)$ and hence $A \cup\{x\} \prec_{m m} A$.

Other well-known examples of orders that satisfy dominance are the lifted orders proposed by Fishburn (1972) and Gärdenfors (1976). Both are frequently used in the context of strategyproofness in elections with tie-breaking (Brandt et al., 2016; Barberà, 2011).

Example 2. Let $X$ be a set, $\leq$ a linear order on $X$ and $\mathcal{X} \subseteq \mathcal{P}(X) \backslash\{\emptyset\}$. Then, the so-called Fishburn extension $\preceq_{f}$ is defined by $A \preceq_{f} B$ if all of the following conditions hold:

- $x<y$ for all $x \in A \backslash B$ and $y \in A \cap B$,

- $y<z$ for all $y \in A \cap B$ and $z \in B \backslash A$,

- $x<z$ for all $x \in A \backslash B$ and $z \in B \backslash A$.

Observe that the first two conditions imply the third unless $A \cap B=\emptyset$. On $\mathcal{T}_{\text {oy }}$ Fishburn's extension looks as follows:

$$
\begin{aligned}
&\{2\} \prec_{f}\{2,4\} \prec_{f}\{4\} ;\{2\} \prec_{f}\{3,4\} \prec_{f}\{4\} ; \\
&\{1,2,4\} \prec_{f}\{4\} ;\{1,4\} \prec_{f}\{4\} ;\{1,2,4\} \prec_{f}\{2,4\} .
\end{aligned}
$$

We claim that $\preceq_{f}$ satisfies dominance. Assume $A, A \cup\{x\} \in \mathcal{X}$ and $\max (A)<x$. Then, $A \backslash(A \cup\{x\})=\emptyset$ and $y<x$ for all $y \in A \cap(A \cup\{x\})=A$. Hence $A \prec_{f} A \cup\{x\}$. The case $x<\min (A)$ is analogous.

The so-called Gärdenfors' extension $\preceq_{g}$ is defined by $A \preceq_{g} B$ if one of the following holds

- $A \subseteq B$ and $x<y$ for all $x \in A$ and $y \in B \backslash A$,

- $B \subseteq A$ and $x<y$ for all $x \in A \backslash B$ and $y \in B$,

- Neither $A \subseteq B$ nor $B \subseteq A$ and $x<y$ for all $x \in A \backslash B$ and $y \in B \backslash A$.

It is known that Gärdenfors' extension is a superset of Fishburn's extension. This means, $A \prec_{f} B$ implies $A \prec_{g} B$ for every $A, B \in \mathcal{X}$. Therefore, it follows directly that Gärdenfors' extension satisfies dominance. On $\mathcal{T}_{\text {oy }}$ Gärdenfors' extension adds to Fishburn's extension the following preferences:

$$
\{1,4\} \prec_{g}\{2,4\} \prec_{g}\{3,4\} ;\{1,2,4\} \prec_{g}\{3,4\}
$$

The second axiom that we consider is called independence. 


\section{Independence}

For all $A, B \in \mathcal{X}$ and all $x \in X \backslash(A \cup B)$, such that $A \cup\{x\}, B \cup\{x\} \in \mathcal{X}$ :

$$
A \prec B \text { implies } A \cup\{x\} \preceq B \cup\{x\} \text {. }
$$

A relation $\preceq$ on $\mathcal{T}_{\text {oy }}$ that satisfies independence must set $\{1,2,4\} \preceq\{1,4\}$ if it contains $\{2,4\} \prec\{4\}$ and $\{1,4\} \preceq\{1,2,4\}$ if it contains $\{4\} \prec\{2,4\}$. As dominance implies $\{2,4\} \prec\{4\}$, dominance and independence together imply $\{1,2,4\} \preceq\{1,4\}$.

Independence is a natural monotonicity axiom that states that if we add the same element $x$ to two sets $A$ and $B$ where $B$ is strictly preferred to $A$, then $B \cup\{x\}$ must be at least weakly preferred to $A \cup\{x\}$. Observe that in contrast to dominance a 'set-based' version of independence would be much stronger that the studied version even if $\mathcal{X}=\mathcal{P}(X) \backslash\{\emptyset\}$. Indeed the definition of independence explicitly includes the possibility that adding one element may already be enough to equalize $A$ and $B$, in which case independence can not be applied again. Therefore, we will not considering 'set-independence'.

Independence is often a very desirable property under the conjunctive interpretation, for example if sets are bundles of objects that are compared according to their overall quality according to some additive utility (Kraft et al., 1959). Indeed, if we define an order based on the sums of utilities, that order satisfies independence.

In this sense there is some tension between the motivations for dominance and independence. Nevertheless, there are cases where both axioms are natural desiderata. These cases are often characterized by the fact that all elements may influence the quality of a set but the extent of this influence is unknown or unknowable. An example under the disjunctive interpretation for such a situation is choice under complete uncertainty:

Example 3. Consider a situation where an agent can perform actions $a_{1}, \ldots, a_{k}$ for which he knows the (set of) possible outcomes but he is not able or not willing to determine the (approximate) probability of each outcome. Such a situation can be modeled as a family of outcomes $X=\left\{o_{1}, o_{2}, \ldots, o_{l}\right\}$ and a function $O:\left\{a_{1}, \ldots, a_{k}\right\} \rightarrow \mathcal{P}(X) \backslash\{\emptyset\}$ that maps every action to the set of possible outcomes of that action. If we assume that the agent has preferences over the set of possible outcomes $X$ that can be modeled as a linear order, the problem of ranking the different actions can be modeled as an order lifting problem. Under this interpretation the extension rule (see below), dominance and independence are usually considered natural desiderata (Bossert et al., 2000; Barberà et al., 2004).

In voting, a comparable situation appears if ties are broken by an unknown chairman. ${ }^{6}$ Similarly, situations exist under the conjunctive interpretation in which it is unclear how much each object contributes to the quality of the set, for example, in voting when sets represent an elected committee in which each member has an (a priori) unknown influence.

6. Which axioms or order are most appropriate in this setting also depends on the risk tolerance of the voters. Very risk averse and undeceive voters might be modeled using Fishburn's extension (Brandt et al., 2018). For agents with other risk profiles $\preceq_{p m m}$, a lexicographic order or something else might be more appropriate. 
In contrast to dominance, independence on its own does not require any preferences. In other words, the empty preference relation always satisfies independence. The weak order defined in Example 1 does not satisfy independence. ${ }^{7}$

Example 4. Let $\preceq_{m m}$ be the weak order defined in Example 1. Consider $X=\{1,2,3,4\}$, $\mathcal{X}=\mathcal{P}(X) \backslash\{\emptyset\}$ and let $\leq$ be the natural linear order on $X$. Then, $\{2\} \prec_{m m}\{1,3\}$ but $\{1,3,4\} \prec_{m m}\{2,4\}$. Therefore, $\preceq_{m m}$ does not satisfy independence.

Furthermore, neither Fishburn's nor Gärdenfors' extension satisfy independence. For example on $\mathcal{T}_{\text {oy }}$ both extensions set $\{2,4\} \prec\{4\}$ but for both $\{1,2,4\}$ and $\{1,4\}$ are incomparable. This violates independence. However, it is possible to define a maxminbased preorder that satisfies dominance and independence together.

Example 5. It can be checked that the following maxmin-based preorder satisfies dominance and independence on every family of sets. We define $\preceq_{p m m}$ by $A \preceq_{p m m} B$ for $A, B \in \mathcal{X}$ if

$$
\max (A) \leq \max (B) \text { and } \min (A) \leq \min (B) .
$$

This relation is obviously reflexive. Furthermore, because $\leq$ is transitive, $\preceq_{p m m}$ is also transitive. Therefore, $\preceq_{p m m}$ is a preorder. We leave it to the reader to check that $\prec_{p m m}$ additionally satisfies dominance and independence.

It turns out that for $\mathcal{X}=\mathcal{P}(X) \backslash\{\emptyset\}$ this is the minimal preorder that satisfies dominance and independence, i.e., every preoder on $\mathcal{P}(X) \backslash\{\emptyset\}$ that satisfies both axioms is an extension of $\preceq_{p m m}$. We observe that $\preceq_{p m m}$ is not total as, for example, $\{1,3\}$ and $\{2\}$ are incomparable.

This raises the question if it is also possible to define a weak order that satisfies both dominance and independence. In 1984 Yakar Kannai and Bezalel Peleg proved in a seminal paper that this is, in general, not possible (Kannai \& Peleg, 1984). To be more precise, they showed that there is no weak order that satisfies both axioms for $\mathcal{X}=\mathcal{P}(X) \backslash\{\emptyset\}$ if $|X| \geq 6$. We will deal with this result in detail in Section 2.3. Before, we introduce a strengthening of independence called strict independence that requires that adding the same element to two sets does not change a strict preference.

\section{Strict Independence}

For all $A, B \in \mathcal{X}$ and for all $x \in X \backslash(A \cup B)$, such that $A \cup\{x\}, B \cup\{x\} \in \mathcal{X}$ :

$$
A \prec B \text { implies } A \cup\{x\} \prec B \cup\{x\} \text {. }
$$

The effect of strict independence on $\mathcal{T}_{\text {oy }}$ is very similar to the effect of independence. A relation $\preceq$ on $\mathcal{T}_{\text {oy }}$ that satisfies strict independence must set $\{1,2,4\} \prec\{1,4\}$ if it contains $\{2,4\} \prec\{4\}$ and $\{1,4\} \prec\{1,2,4\}$ if it contains $\{4\} \prec\{2,4\}$. Dominance implies $\{2,4\} \prec$ $\{4\}$, hence dominance and strict independence together imply $\{1,2,4\} \prec\{1,4\}$.

7. Barberà et al. (2004), following Bossert et al. (2000), falsely claim that the order defined in Example 1 can be characterized by simple dominance, independence and two other axioms. Arlegi (2003) was the first to point out that this is not the case, because $\preceq_{m m}$ does not satisfy independence. He also provided a different axiomatic characterization of this order. 
Clearly, any relation that satisfies strict independence also satisfies independence. Furthermore, any antisymmetric relation that satisfies independence automatically satisfies strict independence. We observe that, like in the case of dominance, a 'set-based' formulation of strict independence would coincide with the given definition if $\mathcal{X}=\mathcal{P}(X) \backslash\{\emptyset\}$. However, strict independence is already a very strong axiom if defined as above. Moreover, it seems natural to define independence and strict independence similarly. Therefore, we will focus on the formulation of strict independence given above and leave a study of 'set-strict-independence' to future work.

Similarly to independence, this is a desirable property, for example, whenever sets should be ranked according to some additive utility. However, it is a significantly stronger axiom and is not satisfied by the preorder $\preceq_{p m m}$ defined in Example 5 .

Example 6. Let $\preceq_{p m m}$ be the preorder defined in Example 5. Furthermore, let $X=$ $\{1,2,3\}$, let $\leq$ be the natural order on $X$ and $\mathcal{X}=\mathcal{P}(X) \backslash\{\emptyset\}$. Then, $\preceq_{p m m}$ does not satisfy strict independence with respect to $\leq$. For example $\{1\} \prec_{p m m}\{1,2\}$ but $\{1,3\} \nprec_{\text {pmm }}$ $\{1,2,3\}$.

Indeed, Salvador Barberà and Prasanta Pattanaik have shown that no preorder can satisfy dominance and strict independence if $|X| \geq 3$ (Barberà \& Pattanaik, 1984). We will discuss this result in more detail in Section 2.3. There are, however, important examples of lifted orders that always satisfy strict independence, like the very well-known lexicographic order. This order is a generalization of the way that words are ordered in a lexicon based on the alphabetical order of the letters (Fishburn, 1974).

One main axiom remains, the so-called extension rule.

\section{The Extension Rule}

For all $x, y \in X$, such that $\{x\},\{y\} \in \mathcal{X}$ :

$x<y$ implies $\{x\} \prec\{y\}$.

In $\mathcal{T}_{\text {oy }}$ the extension rule implies only $\{2\} \prec\{4\}$. In some sense, the extension rule (or just extension for short) is the most basic axiom considered in this paper. It states that the singleton sets in $\mathcal{X}$ need to be ordered the same way as the elements of $X$. In most scenarios, this is a necessary requirement for the lifted order to be acceptable. However, there are exceptions. For example under one interpretation called "freedom of choice" 8 it could be argued that all singletons should be rated equally (see e.g. Pattanaik \& Xu, 1990). If one has to rank the whole powerset i.e., if we assume $\mathcal{X}=\mathcal{P}(X) \backslash\{\emptyset\}$, then the extension rule is implied by dominance for every transitive relation as $\{x\} \prec\{x, y\} \prec\{y\}$ is implied by dominance for all $x, y \in X$ such that $x<y$. However, if we drop the assumption that $\mathcal{X}=\mathcal{P}(X) \backslash\{\emptyset\}$ then there are families of sets on which we can define an order that satisfies dominance and strict independence without satisfying the extension rule. Indeed, Maly et al. (2019) have shown that there are families of sets where dominance and independence can be jointly satisfied with respect to a linear order $\leq$ but dominance, independence and the extension rule are incompatible with respect to $\leq$.

8. For an explanation of this interpretation see either Pattanaik and Xu (1990) or Barberà et al. (2004). 
We observe that the four main axioms, if they are compatible, do not necessarily characterize a unique order. For example both of the following linear orders on $\mathcal{T}_{\text {oy }}$ satisfy all our main axioms.

$$
\begin{aligned}
& \{2\} \prec\{1,2,4\} \prec\{1,4\} \prec\{2,4\} \prec\{3,4\} \prec\{4\}, \\
& \{1,2,4\} \prec\{2\} \prec\{2,4\} \prec\{3,4\} \prec\{1,4\} \prec\{4\} .
\end{aligned}
$$

We finish this section with two important observations about our main axioms that follows directly from their definition.

Observation 7. Let $X$ be a set, $\leq$ a linear order on $X$ and $\mathcal{X} \subseteq \mathcal{P}(X) \backslash\{\emptyset\}$. Furthermore, let $\preceq$ be a relation on $\mathcal{X}$, let $\mathcal{Y} \subseteq \mathcal{X}$ be a subset of $\mathcal{X}$ and let $\preceq \mathcal{Y}$ be the restriction of $\preceq$ to $\mathcal{Y}$. Then, if $\preceq$ satisfies any of our main axioms with respect to $\leq$ then $\preceq \mathcal{Y}$ must satisfy the same axioms with respect to $\leq$.

Proof. All four axioms are universal statements about the ordered set $(\mathcal{X}, \preceq)$. Hence, if they are true for a model $(\mathcal{X}, \preceq)$ they are also true for all its submodels $(\mathcal{Y}, \preceq \mathcal{Y})$, i.e., for all tuples $(\mathcal{Y}, \preceq \mathcal{Y})$ such that $\mathcal{Y} \subseteq \mathcal{X}$ and $x \preceq \mathcal{Y} y$ if and only if $x \prec y$ for all $x, y \in \mathcal{Y}$.

Furthermore, we observe that all our main axioms are symmetric in the following sense.

Lemma 8. Let $X$ be a set, $\leq$ a linear order on $X$ and $\mathcal{X} \subseteq \mathcal{P}(X) \backslash\{\emptyset\}$. Furthermore, let $\prec$ be a order on $\mathcal{X}$. Then, $\prec$ satisfies dominance with respect to a linear order $\leq$ if and only if $\prec^{-1}$ satisfies dominance with respect to $\leq^{-1}$. Here, $R^{-1}$ denotes the inverse of a relation $R$. The same holds for the extension rule, independence and strict independence.

Proof. Let $\preceq$ be an order on $\mathcal{X}$ that satisfies dominance with respect to $\leq$. We claim that $\preceq^{-1}$ satisfies dominance respect to $\leq^{-1}$. Assume $A, A \cup\{x\} \in \mathcal{X}$, then

$$
\forall y \in A\left(y<^{-1} x\right)
$$

implies

$$
\forall y \in A(y>x),
$$

which, by assumption, implies $A \succ A \cup\{x\}$ and hence $A \prec^{-1} A \cup\{x\}$. Similarly,

$$
\forall y \in A\left(x<^{-1} y\right)
$$

implies $A \cup\{x\} \prec^{-1} A$. The argument for the extension rule is similar.

Now, assume $\preceq$ is an order on $\mathcal{X}$ that satisfies strict independence with respect to $\leq$. Then, we claim that $\preceq^{-1}$ satisfies strict independence with respect to $\leq^{-1}$. Assume $A, B, A \cup\{x\}, B \cup\{x\} \in \mathcal{X}$ and $A \prec^{-1} B$. Then, $A \succ B$ and hence, as $\prec$ satisfies strict independence by assumption, $A \cup\{x\} \succ B \cup\{x\}$ which implies $A \cup\{x\} \prec^{-1} B \cup\{x\}$. The argument for independence is the same.

\subsection{Impossibility Results}

As it turns out, it is impossible to jointly satisfy our main axioms, as was shown in two impossibility results by Kannai and Peleg (1984) and Barberà and Pattanaik (1984). Let us first discuss Kannai and Peleg's result in more detail. 


\section{Theorem (Kannai \& Peleg, 1984)}

Let $X$ be a set such that $|X| \geq 6$. Furthermore, let $\leq$ be a linear order on $X$ and $\mathcal{X}=\mathcal{P}(X) \backslash\{\emptyset\}$. Then, there is no weak order on $\mathcal{X}$ that satisfies dominance and independence with respect to $\leq$.

This result is, in several ways, tight. First of all, we cannot drop the requirement that $\preceq$ is total, as we have seen in Example 5 that it is always possible to define a preorder that satisfies dominance and independence.

Furthermore, the assumption $\mathcal{X}=\mathcal{P}(X) \backslash\{\emptyset\}$ is also, in some sense, tight if $|X|=6$. We only have to remove one set from $\mathcal{X}$ to make dominance and independence compatible, though it has to be the right one. For example, the proof of Kannai and Peleg's theorem never mentions any set containing $\max (X)$ and $\min (X)$ at the same time. Therefore, removing such a set will not make dominance and independence compatible. However, removing the set containing the smallest and the second smallest element of $X$ suffices to make dominance, independence and additionally the extension rule jointly satisfiable. We formulate the result w.l.o.g. for $X=\{1,2,3,4,5,6\}$.

Proposition 9 (Maly et al., 2019). Let $X$ be $\{1,2,3,4,5,6\}$. Furthermore, let $\leq$ be the natural linear order on $X$ and $\mathcal{X}=\mathcal{P}(X) \backslash\{\emptyset,\{1,2\}\}$. Then, there is a weak order on $\mathcal{X}$ that satisfies dominance, independence and the extension rule with respect to $\leq$.

Finally, the requirement $|X| \geq 6$ is tight as dominance and independence can be jointly satisfied if $|X| \leq 5$. This follows directly from Proposition 9 but was already proven much earlier by Bandyopadhyay (1988).

Proposition 10 (Bandyopadhyay, 1988). Let $X$ be a set such that $|X| \leq 5$. Furthermore, let $\leq$ be a linear order on $X$ and $\mathcal{X}=\mathcal{P}(X) \backslash\{\emptyset\}$. Then, there is always a weak order on $\mathcal{X}$ that satisfies dominance and independence with respect to $\leq$.

While dominance and independence are incompatible for total orders, it turns out that dominance and strict independence are incompatible already for partial orders. This was proven by Salvador Barberà and Prasanta Pattanaik shortly after Kannai and Peleg published their result (Barberà \& Pattanaik, 1984). Additionally, Barberà and Pattanaik's result requires only a smaller set of elements. Finally, their result also holds if dominance is replaced by a weaker axiom called simple dominance.

Axiom 11 (Simple Dominance). For all $x, y \in X$, such that $\{x\},\{y\},\{x, y\} \in \mathcal{X}$ and $x<y$ :

$$
\{x\} \prec\{x, y\} \prec\{y\} .
$$

Clearly, dominance implies simple dominance on all families of sets. Furthermore, Barberà and Pattanaik (1984) have shown that simple dominance and independence can be jointly satisfied. In contrast, simple dominance and strict independence are incompatible, as was shown in the same paper by Barberà and Pattanaik (1984). 


\section{Theorem (Barberà \& Pattanaik, 1984)}

Let $X$ be a set such that $|X| \geq 3$. Furthermore, let $\leq$ be a linear order on $X$ and $\mathcal{X}=\mathcal{P}(X) \backslash\{\emptyset\}$. Then, there is no binary relation on $\mathcal{X}$ that satisfies simple dominance and strict independence with respect to $\leq$.

It is easy to see that the condition $|X| \geq 3$ is minimal as $\{1\} \prec\{1,2\} \prec\{2\}$ satisfies dominance and strict independence. Furthermore, it is again the case that removing one element from $\mathcal{P}(X) \backslash\{\emptyset\}$ for $|X|=3$ suffices to make dominance, strict independence and the extension rule compatible.

Example 12. Consider $\mathcal{X}=\mathcal{P}(X) \backslash\{\emptyset,\{3\}\}$. Then, the following linear order satisfies dominance and strict independence:

$$
\{1\} \prec\{1,2\} \prec\{1,3\} \prec\{1,2,3\} \prec\{2\} \prec\{2,3\} .
$$

It is straightforward to check that dominance is satisfied. For strict independence, we have to consider all pairs $A, B \in \mathcal{X}$ and all $x \notin A \cup B$ such that $A \cup\{x\}, B \cup\{x\} \in \mathcal{X}$ holds. First assume $x=1$. Then, $A, B$ must be $\{2\}$ and $\{2,3\}$. We see that $\{2\} \prec\{2,3\}$ and $\{1,2\} \prec\{1,2,3\}$ hold, hence strict independence is satisfied for this pair. Now assume $x=2$. Then, $A, B$ must be $\{1\}$ and $\{1,3\}$. Now, as $\{1\} \prec\{1,3\}$ and $\{1,2\} \prec\{1,2,3\}$ hold, strict independence is also satisfied in this case. Finally, consider the case that $x=3$. Then, $A$ and $B$ must be $\{1\},\{1,2\}$ or $\{2\}$. Now, we have $\{1\} \prec\{1,2\} \prec\{2\}$ and $\{1,3\} \prec$ $\{1,2,3\} \prec\{2,3\}$. Hence, strict independence is satisfied.

\subsection{Three Types of Orderability}

Given a set $X$, a family $\mathcal{X} \subseteq \mathcal{P}(X) \backslash\{\emptyset\}$ and a set of axioms $\mathcal{A}$, we can distinguish three degrees to which the axioms in $\mathcal{A}$ can be compatible on $\mathcal{X}$. First, they can be compatible for at least one linear order on $X$. Second, they can be compatible with respect to a specific linear order $\leq$ on $X$. Finally, they can be compatible for every linear order on $X$. We can view these as a property of a family of sets with respect to a set of axioms. The following definitions were first introduced by Maly, Truszczyński, and Woltran (2018).

Definition 13. Let $X$ be a set and $\mathcal{X} \subseteq \mathcal{P}(X) \backslash\{\emptyset\}$. Furthermore, let $\mathcal{A}$ be a set of axioms. Then, we say that $\mathcal{X}$ is ...

- ... weakly orderable with respect to $\mathcal{A}$ if there is a linear order $\leq^{*}$ on $X$ such that there is a weak order on $\mathcal{X}$ that satisfies all axioms in $\mathcal{A}$ with respect to $\leq^{*}$.

- ... $\leq$-orderable with respect to $\mathcal{A}$ for a linear order $\leq$ on $X$, if there is a weak order on $\mathcal{X}$ that satisfies all axioms in $\mathcal{A}$ with respect to $\leq$.

- ... strongly orderable with respect to $\mathcal{A}$ if for all linear orders $\leq^{*}$ on $X$ there is a weak order on $\mathcal{X}$ that satisfies all axioms in $\mathcal{A}$ with respect to $\leq^{*}$.

For convenience, we will define a shorthand for orderability with respect to (sub)sets of our main axioms. 
Definition 14. Let $X$ be a set, $\leq$ a linear order on $X$ and $\mathcal{X} \subseteq \mathcal{P}(X) \backslash\{\emptyset\}$. Assume $\mathcal{X}$ is $\leq$-orderable with respect to a set of axioms $\mathcal{A}$. Then, we say $\mathcal{X}$ is...

- ... $\leq-D I$-orderable if $\mathcal{A}$ consists of dominance and independence.

- . . $\leq-D I E$-orderable if $\mathcal{A}$ consists of dominance, independence and the extension rule.

- .. $\leq-D I^{S}$-orderable if $\mathcal{A}$ consists of dominance and strict independence.

- . . $\leq-D I^{S} E$-orderable if $\mathcal{A}$ consists of dominance, strict independence and the extension rule.

We use the same notation also for strong and weak orderability.

The following example demonstrates the use of these shorthands.

Example 15. Let $X=\{1,2,3\}$ and $\mathcal{X}=\mathcal{P}(X) \backslash\{\emptyset,\{3\}\}$. Furthermore, let $\leq^{*}$ be the natural linear order $1<^{*} 2<^{*} 3$. Then, $\leq^{*}$ can be lifted to a linear order on $\mathcal{X}$ that satisfies dominance and strict independence, as we have seen in Example 12. Therefore, $\mathcal{X}$ is $\leq^{*}-D I^{S}$-orderable and also weakly $D I^{S}$-orderable. On the other hand, if we consider the linear order $1<^{\prime} 3<^{\prime} 2$ on $X$, then no linear order on $\mathcal{X}$ satisfies dominance and strict independence with respect to $\leq^{\prime}$. This is because the proof of Barberà and Pattanaik's impossibility result does not mention $\{2\}$, which has the same position under the natural linear order as $\{3\}$ has under $\leq^{\prime}$. Therefore, $\mathcal{X}$ is not strongly $D I^{S}$-orderable. If we consider independence instead of strict independence, then Proposition 10 implies that $\mathcal{X}$ is strongly $D I E$-orderable because it has less than 6 elements.

The classical works on ranking sets of objects as surveyed by Barberà et al. (2004) do not distinguish different kinds of orderability, because they only consider the case $\mathcal{X}=$ $\mathcal{P}(X) \backslash\{\emptyset\}$. In this case all three aforementioned types of orderability coincide, because for every permutation $\pi$ of $X$ we have $\pi(\mathcal{P}(X) \backslash\{\emptyset\})=\mathcal{P}(X) \backslash\{\emptyset\}$.

\subsection{Reverse Independence and Reverse Strict Independence}

In this section, we discuss two additional axioms, which are obtained by reversing the direction of independence or strict independence. These will be useful when proving our main results.

Axiom 16 (Reverse independence). For all $A, B \in \mathcal{X}$ and for all $x \in X \backslash(A \cup B)$ such that $A \cup\{x\}, B \cup\{x\} \in \mathcal{X}:$

$$
A \cup\{x\} \prec B \cup\{x\} \text { implies } A \preceq B .
$$

Axiom 17 (Reverse strict independence). For all $A, B \in \mathcal{X}$ and for all $x \in X \backslash(A \cup B)$ such that $A \cup\{x\}, B \cup\{x\} \in \mathcal{X}$ :

$$
A \cup\{x\} \prec B \cup\{x\} \text { implies } A \prec B \text {. }
$$

These axioms are very similar to independence and strict independence, intuitively as well as technically. One could argue that they are a slightly less natural formulation of the same monotonicity idea. Independence is equivalent to its reverse counterpart for total 
orders but both versions differ for partial orders. For example, if $X=\{1,2,3\}$ and $\mathcal{X}=$ $\{\{1\},\{2\},\{1,3\},\{2,3\}\}$ then the partial order only containing the preference $\{1\} \prec\{2\}$ does satisfy reverse independence but not independence. On the other hand, the partial order only containing the preference $\{1,3\} \prec\{2,3\}$ satisfies independence but not reverse independence. Similarly, strict independence is equivalent to its reverse counterpart for linear orders but both versions differ for non-linear orders.

Proposition 18. Let $X$ be a set, $\leq$ a linear order on $X$ and $\mathcal{X} \subseteq \mathcal{P}(X) \backslash\{\emptyset\}$. Then, a total relation on $\mathcal{X}$ satisfies independence if and only if it satisfies reverse independence. $A$ total, antisymmetric relation satisfies strict independence if and only if it satisfies reverse strict independence.

Proof. Let $\preceq$ be a total relation on $\mathcal{X}$. Furthermore, assume that $A, B, A \cup\{x\}$ and $B \cup\{x\}$ are in $\mathcal{X}$. We show that reverse independence implies independence. The other direction is analogous. Assume, that $A \prec B$ and $\preceq$ satisfies reverse independence. Then, by totality, we must have either $A \cup\{x\} \preceq B \cup\{x\}$ or $B \cup\{x\} \prec A \cup\{x\}$. In the second case, reverse independence would imply $B \prec A$ which contradicts our assumption that $A \prec B$ holds. Hence. $A \prec B$ always implies $A \cup\{x\} \preceq B \cup\{x\}$. In other words, $\preceq$ satisfies independence.

Now, let $\preceq$ be a total, antisymmetric relation on $\mathcal{X}$. If $\preceq$ satisfies reverse strict independence, it must, by definition, also satisfy reverse independence. As we have proven above, this implies that $\preceq$ satisfies independence. Now, because $\preceq$ is an antisymmetric relation that satisfies independence it must, by definition, also satisfy strict independence. The other direction is analogous.

We observe that dominance is "more compatible" with reverse strict independence than with strict independence.

Observation 19. Let $X$ be a set with three elements, $\leq$ a linear order on $X$ and $\mathcal{X}=$ $\mathcal{P}(X) \backslash\{\emptyset\}$. Then, there exists a total order on $\mathcal{X}$ satisfying dominance and reverse strict independence with respect to $\leq$.

Proof. We assume w.l.o.g. that $X=\{1,2,3\}$ and $\leq$ is the natural linear order on $X$. Then, we claim that

$$
\{1\} \prec\{1,2\} \prec\{1,2,3\} \sim\{1,3\} \prec\{2\} \prec\{2,3\} \prec\{3\} .
$$

is a total order that satisfies dominance and reverse strict independence. We have seen in Example 12 that the restriction of $\preceq$ to $\mathcal{X} \backslash\{3\}$ is a linear order that satisfies dominance and strict independence, hence also reverse strict independence. Therefore, we only need to look at applications of dominance and reverse strict independence that involve $\{3\}$. Clearly, the only applications of dominance involving $\{1,3\}$ are $\{1,3\} \prec\{3\}$ and $\{2,3\} \prec\{3\}$, both of which are satisfied. Now, consider the case that $A \cup\{x\} \prec B \cup\{x\}$ holds for $A, B \in \mathcal{P}(X) \backslash\{\emptyset\}$ and $x \notin A \cup B$. Clearly $A \cup\{x\}=\{3\}$ or $B \cup\{x\}=\{3\}$ is not possible. Therefore, we can assume that and $A=\{3\}$ or $B=\{3\}$. Assume first that $x=2$. Then $B=\{3\}$ and $A=\{1\}$ or $A=\{1,3\}$. In both cases, reverse strict independence is satisfied. Now assume that $x=1$. Then $B=\{3\}$ and $A=\{2\}$. For this pair reverse strict independence is also satisfied. 
On the other hand, we have seen that reverse strict independence implies independence for total relations. Hence, there is no weak order that satisfies dominance and reverse independence if $\mathcal{X}=\mathcal{P}(X) \backslash\{\emptyset\}$ for any set $X$ with $|X| \geq 6$.

\section{Complexity Results}

In this section we present our results. We study several problems related to $\leq$-orderability, strong orderability and weak orderability. Together, these results give a nearly complete picture of the complexity of deciding whether dominance and (strict) independence are jointly satisfiable for a given family.

In the following, we formally define the studied problems and describe the structure of this section. The problems are named according to the following system: First, it is indicated whether the problem concerns strong, weak or $\leq$-orderability. The last case is specified by the absence of the words strong and weak. Then, the set of axioms in defined using the same notation as for orderability. Finally, the letters LO, WO, or PO declare whether we expect the lifted order to be linear, weak or partial. Hence, for example, the problem of deciding whether a family $\mathcal{X}$ is $\leq-D I^{S}$-orderable is called $D I^{S}$-WO-OrderabiLiTy.

In the beginning of the chapter, we study $\leq$-orderability and strong orderability. First we consider the problem of lifting a linear order on $X$ to a linear order on $\mathcal{X} \subseteq \mathcal{P}(X) \backslash\{\emptyset\}$. In this setting, independence and strict independence coincide so we study this problem only for strict independence. This gives us four problems to study. Two of these problems are:

$D I^{S}$-LO-ORDERABILITY

Input: $\quad$ A set $X$, a family of sets $\mathcal{X} \subseteq \mathcal{P}(X)$ and a linear order $\leq$ on $X$.

Question: Is there a linear order on $\mathcal{X}$ that satisfies dominance and strict independence with respect to $\leq$ ?

Strong $D I^{S}$-LO-ORDERABILITy

Input: $\quad$ A set $X$ and a family of sets $\mathcal{X} \subseteq \mathcal{P}(X)$.

Question: Is there for every linear order $\leq$ on $X$ a linear order on $\mathcal{X}$ that satisfies dominance and strict independence with respect to $\leq$ ?

The other two problems are obtained by adding the extension rule to the requirements.

$D I^{S} E$-LO-ORDERABILITY

Input: $\quad$ A set $X$, a family of sets $\mathcal{X} \subseteq \mathcal{P}(X)$ and a linear order $\leq$ on $X$.

Question: Is there a linear order on $\mathcal{X}$ that satisfies dominance, strict independence and the extension rule with respect to $\leq$ ?

Strong $D I^{S} E$-LO-Orderability

Input: $\quad$ A set $X$ and a family of sets $\mathcal{X} \subseteq \mathcal{P}(X)$.

Question: Is there for every linear order $\leq$ on $X$ a linear order on $\mathcal{X}$ that satisfies dominance, strict independence and the extension rule with respect to $\leq$ ?

We first prove that Strong $D I^{S}$-LO-Orderability is NP-hard (Proposition 21) before we improve the result to $\Sigma_{2}^{p}$-completeness in Theorem 29. The reduction used for this result will immediately also prove the NP-completeness of $D I^{S}$-LO-OrDERABILITY 
(Corollary 22). Furthermore, we show that adding the extension rule does not change the complexity of the problems, i.e., we show that Strong $D I^{S} E$-LO-OrderabiLity is NP-hard and $D I^{S} E$-LO-ORDERABILITY is NP-complete (Corollary 23).

Next, we study the complexity of lifting a linear order on $X$ to a total, but not necessarily linear, order on $\mathcal{X}$ that satisfies dominance and strict independence. This gives us again two problems:

$D I^{S}$-WO-ORDERABILITY

Input: $\quad$ A set $X$, a linear order $\leq$ on $X$ and a family of sets $\mathcal{X} \subseteq \mathcal{P}(X)$.

Question: Is $\mathcal{X} \leq-D I^{S}$-orderable?

Strong $D I^{S}$-WO-ORDERABILITy

Input: $\quad$ A set $X$ and a family of sets $\mathcal{X} \subseteq \mathcal{P}(X)$.

Question: Is $\mathcal{X}$ strongly $D I^{S}$-orderable?

Modifying the reduction used in Proposition 21 we additionally prove that $D I^{S}$-WOOrderABILITY is NP-complete (Theorem 25). A further modification of the reduction used for Proposition 21, shows that Strong $D I^{S}$-WO-Orderability is $\Pi_{2}^{p}$-complete (Theorem 27). As before we can define two more problems by adding the extension rule to the requirements, $D I^{S} E$-WO-Orderability and Strong $D I^{S} E$-WO-Orderability. The same complexity results also hold for these problems i.e., $D I^{S} E$-WO-OrderabiLity is NP-complete and Strong $D I^{S} E$-WO-Orderability is $\Pi_{2}^{p}$-complete (Corollary 26 and 28).

To conclude the section on lifting to a total order, we study the complexity of lifting a linear order on $X$ to a weak order on $\mathcal{X}$ that satisfies dominance and independence. This gives us the following problems:

Strong DI-WO-ORDERABILITY

Input: $\quad$ A set $X$ and a family of sets $\mathcal{X} \subseteq \mathcal{P}(X)$.

Question: Is $\mathcal{X}$ strongly $D I$-orderable?

DI-WO-ORDERABILITY

Input: $\quad$ A set $X$, a linear order $\leq$ on $X$ and a family of sets $\mathcal{X} \subseteq \mathcal{P}(X)$.

Question: Is $\mathcal{X} \leq-D I$-orderable?

Again, we additionally study the problems obtained by adding the extension rule. With another modification of the reduction used before, we can prove that STRONG DI-WOOrderABILITY is $\Pi_{2}^{p}$-complete and that $D I$-WO-ORDERABILITY is NP-complete (Theorem 30). As before, both results hold if we add the extension rule, i.e., Strong DIEWO-ORDERABILITY is $\Pi_{2}^{p}$-complete and DIE-WO-ORDERABILITY is NP-complete (Corollary 31$)$.

The next problem that we consider is the problem of lifting to an partial order. As we have seen in Example 5, it is always possible to find a preorder that satisfies dominance, independence and the extension rule. Therefore, we only consider the problem of lifting a linear order on $X$ to a partial order on $\mathcal{X}$ that satisfies either dominance and strict independence or dominance, strict independence and the extension rule. Maly and Woltran (2017) gave a constructive, polynomial time procedure for constructing a minimal transitive, reflexive binary relation that satisfies dominance and strict independence, resp. 
dominance, strict independence and the extension rule. In this paper, we consider strong orderability with respect to partial orders and show by a reduction from TAUT that the following problems are coNP-complete (Theorem 36).

Strong $D I^{S}$-PO-ORDERABILITY

Input: $\quad$ A set $X$ and a family of sets $\mathcal{X} \subseteq \mathcal{P}(X)$.

Question: Is there for every linear order $\leq$ on $X$ a partial order on $\mathcal{X}$ that satisfies dominance and strict independence with respect to $\leq$ ?

Strong $D I^{S} E$-PO-Orderability

Input: $\quad$ A set $X$ and a family of sets $\mathcal{X} \subseteq \mathcal{P}(X)$.

Question: Is there for every linear order $\leq$ on $X$ a partial order on $\mathcal{X}$ that satisfies dominance, strict independence and the extension rule with respect to $\leq$ ?

Afterwards, we discuss succinctly represented families of sets. We introduce the historical and necessary technical background on problems that are represented by boolean circuits. Then, we show for most of the problems that we studied in this chapter that their complexity increases exponentially if the instances are represented by boolean circuits.

Next, we shift our attention to weak orderability. In this context, we only study strict independence. We leave the question whether the following results also hold for independence for future work. The first problem that we consider is weak $D I^{S}$-orderability.

WEAK $D I^{S}$-WO-ORDERABILITY

Input: $\quad$ A set $X$ and a family of sets $\mathcal{X} \subseteq \mathcal{P}(X)$.

Question: Is $\mathcal{X}$ weakly $D I^{S}$-orderable?

We show with a reduction from BeTweEnNEss that this problem is NP-complete (Theorem 46). A close inspection of the proof shows that this also holds if we additionally require the extension rule and if we require the lifted order to be linear or only partial. This means WEAK $D I^{S}$-PO-Orderability, WeAK $D I^{S}$-LO-OrderabiLity as well as WeAK $D I^{S} E$-PO-Orderability, WeAK $D I^{S} E$-WO-Orderability and WeAK $D I^{S} E$ LO-ORDERABILITY are all NP-complete (see Corollary 47) .

We conclude the paper by exploring if strengthening dominance is a viable way to reduce the complexity of the studied problems. We restrict our attention to $\leq-D I^{S}$-orderability and show that it stays NP-hard for all "reasonable" strengthenings of dominance (Theorem 51). Formally, we say that an axiom $A$ is a reasonable strengthening of dominance if

- $A$ implies dominance and

- $A$ is implied by a very strong axiom called maximal dominance (Axiom 48).

Table 1 summarizes the results proven in this chapter. The [Thm] column specifies the Theorem, Proposition or Corollary in which the result is proven. "known" indicates that a result is folklore. Results marked with $(\star)$ were already shown by Maly and Woltran (2017).

\section{$3.1 \leq-$ Orderability and Strong Orderability}

In this section, we discuss the complexity of several variants of $\leq$-orderability and strong orderability with respect to some subsets of our main axioms. The unifying feature of the 


\begin{tabular}{|c|c|c|c|c|}
\hline Orderability & Dom + Ind & {$[\mathrm{Thm}]$} & Dom + strict Ind & [Thm] \\
\hline$\leq-\mathrm{PO}-$ ord & always & known & $\operatorname{in} P(\star)$ & 35 \\
\hline strong PO-ord. & always & known & coNP-c. $(\dagger)$ & 36 \\
\hline weak PO-ord. & always & known & NP-c. & 47 \\
\hline$\leq-\mathrm{WO}$-ord & NP-c. $(\star)$ & 30 & NP-c. & 25 \\
\hline strong WO-ord. & $\Pi_{2}^{p}$-c. $(\dagger)$ & 30 & $\Pi_{2}^{p}$-c. & 27 \\
\hline weak WO-ord. & open & $\mathrm{n} / \mathrm{a}$ & NP-c. & 46 \\
\hline$\leq$-LO-ord. & NP-c. & 32 & NP-c. $(\star)$ & 22 \\
\hline strong LO-ord. & $\Pi_{2}^{p}$-c. & 32 & $\Pi_{2}^{p}$-c. $(\dagger)$ & 29 \\
\hline weak LO-ord. & open & $\mathrm{n} / \mathrm{a}$ & NP-c. & 47 \\
\hline succ. strong PO-ord. & always & known & coNEXP-c. $(\dagger)$ & 45 \\
\hline succ. $\leq$-WO-ord. & NEXP-c. $(\dagger)$ & 44 & NEXP-c. & 44 \\
\hline succ. strong WO-ord. & NEXP-hard $(\dagger)$ & 44 & NEXP-hard & 44 \\
\hline succ. $\leq-\mathrm{LO}-$ ord. & NEXP-c. & 44 & NEXP-c. $(\dagger)$ & 43 \\
\hline succ. strong LO-ord. & NEXP-hard & 44 & NEXP-hard $(\dagger)$ & 43 \\
\hline
\end{tabular}

Table 1: The complexity of orderability with respect to dominance and (strict) independence. $(\star)$ indicates that the result was already shown by Maly and Woltran (2017). $(\dagger)$ indicates that the result was alredy contained in the conference version of this paper (Maly, 2020a).

problems discussed in this section is the fact that their hardness can be proven by a variation of the same reduction from either $\mathrm{SAT}$ or $\Pi_{2}$-SAT.

\section{$D I^{S}$-LO-ORDERABILITY}

We show first that Strong $D I^{S}$-LO-Orderability is NP-hard, even tough we will improve this result in Corollary 29 by showing that Strong $D I^{S}$-LO-Orderability is $\Pi_{2}^{p}$-complete. This approach allows us to present the simplest form of a reduction from SAT that will be used - with some modifications - to prove several other hardness results in this chapter.

Let us first give an intuitive description of the main ideas of the proof. The goal of the reduction is to encode a $3-\mathrm{CNF} \phi$ as a families of sets $\mathcal{X}$. First the variables in $\phi$ are encoded: Every variable $V_{i}$ in $\phi$ will be encoded by two sets $X_{i}^{\mathrm{t}}$ and $X_{i}^{\mathrm{f}}$. Then, we can equate every linear order $\preceq$ on $\mathcal{X}$ with a truth assignment to the variables in $\phi$ by saying that $V_{i}$ is set to true if $X_{i}^{\mathrm{f}} \prec X_{i}^{\mathrm{t}}$ and $V_{i}$ is set to false if $X_{i}^{\mathrm{t}} \prec X_{i}^{\mathrm{f}}$. Because $\prec$ is a linear order and therefore antisymmetric and total, this defines a complete and consistent truth assignment. Then, we will add sets to $\mathcal{X}$ that lead to a cycle in every linear order $\preceq$ that satisfies dominance and strict independence with respect to $\leq$ if $\preceq$ does not encode a satisfying truth assignment to $\phi$. To achieve this, we will use the following observation: If $\leq$ is a linear order on a set $X$ and $A$ and $B$ are two subsets of $X$ such that

$$
\min (B)<\min (A)<\max (A)<\max (B)
$$


then there always exists a collection of sets $\mathcal{Y}$ such that any linear order $\preceq$ on $\mathcal{Y} \cup\{A, B\}$ that satisfies dominance and strict independence with respect to $\leq$ has to set $A \prec B$. At the same time, there also has to exist a collection of sets $\mathcal{Y}^{*}$ such that any linear order $\preceq$ on $\mathcal{Y}^{*} \cup\{A, B\}$ that satisfies dominance and strict independence with respect to $\leq$ has to set $B \prec A$. Let us illustrate this by an example.

Example 20. Let $X=\{1, \ldots, 5\}$ and let $\leq$ be the natural linear order on $X$. Now consider $A=\{3\}, B=\{2,3,4\}$. Then clearly

$$
\min (B)<\min (A)<\max (A)<\max (B) .
$$

First, we claim that for the following collection

$$
\mathcal{Y}=\{\{1,2,3,4\},\{1,3\},\{1\},\{1,2\},\{1,2,3\}\}
$$

any linear order $\preceq$ on $\mathcal{Y} \cup\{A, B\}$ that satisfies dominance and strict independence with respect to $\leq$ has to set $A \prec B$. Assume for the sake of contradiction that there is a linear order $\preceq$ on $\mathcal{Y} \cup\{A, B\}$ with $B \prec A$ that satisfies dominance and strict independence with respect to $\leq$. Then, strict independence implies

$$
B \cup\{1\}=\{1,2,3,4\} \prec\{1,3\}=A \cup\{1\} .
$$

However, by dominance we have $\{1\} \prec\{1,2\}$ and hence by strict independence and dominance

$$
\{1,3\} \prec\{1,2,3\} \prec\{1,2,3,4\},
$$

a contradiction. On the other hand, it can be checked that

$$
\{1\} \prec\{1,2\} \prec\{1,2,3\} \prec\{1,3\} \prec\{3\} \prec\{1,2,3,4\} \prec\{2,3,4\}
$$

is a linear order on $\mathcal{Y} \cup\{A, B\}$ that satisfies dominance and strict independence with respect to $\leq$.

By a similar argument, we can see that

$$
\mathcal{Y}^{*}=\{\{2,3,4,5\},\{3,5\},\{5\},\{4,5\},\{3,4,5\}\} .
$$

has the property that that any linear order $\preceq$ on $\mathcal{Y}^{*} \cup\{A, B\}$ that satisfies dominance and strict independence with respect to $\leq$ has to set $B \prec A$.

We will use this observation and define the sets encoding variables in a way such that for all $a, b \in\{\mathrm{t}, \mathrm{f}\}$ and $i, j \leq n$, where $n$ is the number of variables in $\phi$, we have either

$$
\min \left(X_{i}^{a}\right)<\min \left(X_{j}^{b}\right)<\max \left(X_{j}^{b}\right)<\max \left(X_{i}^{a}\right)
$$

or

$$
\min \left(X_{j}^{b}\right)<\min \left(X_{i}^{a}\right)<\max \left(X_{i}^{a}\right)<\max \left(X_{j}^{b}\right) .
$$

This can be ensured for example by a construction where every set has a common middle part and a unique minimal and maximal element. Then, for every new set we increase the minimal and decrease the maximal element at the same time (See Figure 2). 


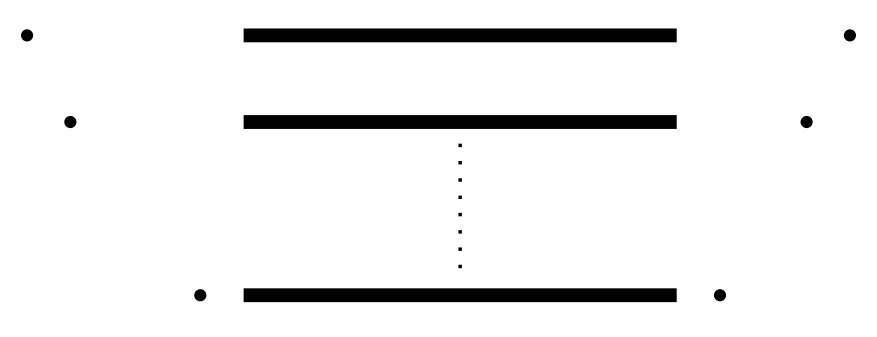

Figure 2: Sets encoding variables.

Then we can enforce any preference we need between sets encoding different variables by adding the correct collection of set. We use this to enforce for every clause preferences that lead to a contradiction whenever no literal in the clause is satisfied. Consider for example the clause $C=x_{1} \vee \neg x_{2}$. Then, we enforce the preferences

$$
X_{1}^{\mathrm{f}} \prec X_{2}^{\mathrm{f}} \text { and } X_{2}^{\mathrm{t}} \prec X_{1}^{\mathrm{t}}
$$

Now consider a linear order $\preceq$ that contains

$$
X_{1}^{\mathrm{t}} \prec X_{1}^{\mathrm{f}} \text { and } X_{2}^{\mathrm{f}} \prec X_{2}^{\mathrm{t}}
$$

and hence encodes an assignment that sets $x_{1}$ to false and $x_{2}$ to true. This assignment does not satisfy $C$ and indeed $\preceq$ contains the following cycle

$$
X_{1}^{\mathrm{f}} \prec X_{2}^{\mathrm{f}} \prec X_{2}^{\mathrm{t}} \prec X_{1}^{\mathrm{t}} \prec X_{1}^{\mathrm{f}}
$$

On the other hand,

$$
X_{1}^{\mathrm{f}} \prec X_{2}^{\mathrm{f}} \prec X_{2}^{\mathrm{t}} \prec X_{1}^{\mathrm{t}}
$$

is a linear order that is compatible with the enforced preferences and encodes the satisfying assignment that sets $x_{1}$ and $x_{2}$ to true.

Using this idea, we can add for every clause sets that lead to a cycle if the clause is not satisfied by the assignment coded by a linear order. The main technical difficultly of the proof will be to implement this approach in a way that ensures that no cycle occurs if $\preceq$ encodes a satisfying truth assignment.

\section{Proposition 21. Strong $D I^{S}$-LO-ORderability is NP-hard.}

Proof. Let $\phi$ be an instance of SAT with $n$ variables and $m$ clauses. We will produce an instance $(X, \mathcal{X})$ of Strong $D I^{S}$-LO-Orderability. Furthermore, we will fix a specific linear order $\leq$ on $X$ such that there is a linear order on $\mathcal{X}$ that satisfies dominance and strict independence with respect to $\leq$ only if $\phi$ is satisfiable. Furthermore, we want to make sure that we can use a satisfying assignment of $\phi$ to construct for any arbitrary linear order $\leq^{\prime}$ on $X$ a linear order $\preceq$ on $\mathcal{X}$ that satisfies dominance and strict independence.

First, we construct the set of elements $X$. For every variable $V_{i}$, the set $X$ contains elements $x_{i, 1}^{-}, x_{i, 2}^{-}, x_{i, 1}^{+}$and $x_{i, 2}^{+}$. These will be used to construct the sets for different variables. Furthermore, it contains for every clause $C_{j}$ variables $z_{j}^{a}, y_{j}^{a}$, $\min _{j}^{a}$ and $\max _{j}^{a}$ for $a \in\{1,2,3\}$. In the following, we will call $\min _{j}^{a}$ and $\max _{j}^{a}$ extremum elements. These will be used to 
ensure that only orders encoding a satisfying assignment to the variables in $C_{j}$ can satisfy dominance and strict independence. Finally, it contains two elements $v_{1}$ and $v_{2}$. These will determine the "orientation" of a linear order $\leq$ on $X$. In general, the order lifting problem is symmetric (see Lemma 8) whereas SAT is not symmetric with respect to truth. To overcome this difficultly, the preference between $v_{1}$ and $v_{2}$ determines if $X_{i}^{\mathrm{f}} \prec X_{i}^{\mathrm{t}}$ means that $V_{i}$ is true or that $V_{i}$ is false. This way, $\leq^{\prime}$ and $\leq^{\prime-1}$ encode the same truth assignment. Next, we fix a linear order $\leq$ that we define by:

$$
\begin{aligned}
& \min _{1}^{1}<\min _{1}^{2}< \cdots<\min _{m}^{3}<x_{1,1}^{-}<x_{1,2}^{-}<\cdots<x_{n, 2}^{-} \\
&<v_{1}<v_{2}<z_{1}^{1}<z_{1}^{2} \cdots<z_{m}^{3}<y_{1}^{1}<y_{1}^{2}<\cdots<y_{m}^{3}< \\
& x_{1,1}^{+}<x_{1,2}^{+}<\cdots<x_{n, 2}^{+}<\max _{1}^{1}<\max _{1}^{2}<\cdots<\max _{m}^{3}
\end{aligned}
$$

We call this the critical linear order. Now, we construct the family $\mathcal{X}$. Our first goal is to ensure that there does not exists a linear order on $\mathcal{X}$ that satisfies dominance and strict independence with respect to $\leq$ if $\phi$ is not satisfiable. In the following, we write $Y:=\left\{x \in X \mid v_{1} \leq x \leq y_{m}^{3}\right\}$. First we add the sets representing the variables of $\phi$. We add for every variable $V_{i}$ sets $X_{i}^{\mathrm{t}}=Y \cup\left\{x_{i, 1}^{-}, x_{i, 1}^{+}\right\}$and $X_{i}^{\mathrm{f}}=Y \cup\left\{x_{i, 2}^{-}, x_{i, 2}^{+}\right\}$. We call these the Class 1 sets and write $C l_{1}$ for the collection of all Class 1 sets.

Now, let $C_{i}$ be a clause with variables $V_{j}, V_{k}$ and $V_{l}$. We want to "enforce" specific preferences between the sets representing $V_{j}, V_{k}$ and $V_{l}$ depending on whether they appear positively or negatively in $C_{i}$. However, this could lead to problems if the same variables also occur in another clause. Therefore, we add what could be considered local instantiations of the sets representing the variables $V_{j}, V_{k}$ and $V_{l}$ :

$$
X_{j}^{\mathrm{t}} \backslash\left\{y_{i}^{1}\right\}, X_{j}^{\mathrm{f}} \backslash\left\{y_{i}^{1}\right\}, X_{k}^{\mathrm{t}} \backslash\left\{y_{i}^{2}\right\}, X_{k}^{\mathrm{f}} \backslash\left\{y_{i}^{2}\right\}, X_{l}^{\mathrm{t}} \backslash\left\{y_{i}^{3}\right\} \text { and } X_{l}^{\mathrm{f}} \backslash\left\{y_{i}^{3}\right\} .
$$

We call these the Class 2 sets and write $C l_{2}$ for the collection of all Class 2 sets. Now, let $\preceq$ be a linear order on $\mathcal{X}$ that satisfies strict independence. Then, it also satisfies reverse strict independence by Proposition 18. By reverse strict independence we know that for $\preceq$ the preference between $X_{j}^{\mathrm{t}} \backslash\left\{y_{i}^{1}\right\}$ and $X_{j}^{\mathrm{f}} \backslash\left\{y_{i}^{1}\right\}$ must be the same as the preference between $X_{j}^{\mathrm{t}}$ and $X_{j}^{\mathrm{f}}$. The same holds for the other two variables. In this sense, these "local instantiations" correctly reflect the truth assignment encoded by any linear order on $\mathcal{X}$, if the linear order satisfies strict independence. On the other hand, any preference between local instantiations of sets representing different variables, say $X_{j}^{\mathrm{t}} \backslash\left\{y_{i}^{1}\right\}$ and $X_{k}^{\mathrm{f}} \backslash\left\{y_{i}^{2}\right\}$ stays local, because $y_{i}^{1} \neq y_{i}^{2}$.

Now, if all variables occur positively in $C_{i}$, we add sets such that $X_{j}^{\mathrm{f}} \backslash\left\{y_{i}^{1}\right\} \prec X_{k}^{\mathrm{t}} \backslash\left\{y_{i}^{2}\right\}$, $X_{k}^{\mathrm{f}} \backslash\left\{y_{i}^{2}\right\} \prec X_{l}^{\mathrm{t}} \backslash\left\{y_{i}^{3}\right\}$ and $X_{l}^{\mathrm{f}} \backslash\left\{y_{i}^{3}\right\} \prec X_{j}^{\mathrm{t}} \backslash\left\{y_{i}^{1}\right\}$ must hold for any order $\preceq$ on $\mathcal{X}$ that satisfies dominance and strict independence with respect to $\leq$. We call this enforcing these preferences. Then, we get a contradiction if $X_{a}^{\mathrm{t}} \backslash\left\{y_{i}^{b}\right\} \prec X_{a}^{\mathrm{f}} \backslash\left\{y_{i}^{b}\right\}$ for all $a \in\{j, k, l\}$, i.e., if $\preceq$ corresponds to a truth assignment where $V_{j}, V_{k}$ and $V_{l}$, are false because

$$
X_{j}^{\mathrm{t}} \backslash\left\{y_{i}^{1}\right\} \prec X_{j}^{\mathrm{f}} \backslash\left\{y_{i}^{1}\right\} \prec X_{k}^{\mathrm{t}} \backslash\left\{y_{i}^{2}\right\} \prec X_{k}^{\mathrm{f}} \backslash\left\{y_{i}^{2}\right\} \prec X_{l}^{\mathrm{t}} \backslash\left\{y_{i}^{3}\right\} \prec X_{l}^{\mathrm{f}} \backslash\left\{y_{i}^{3}\right\} \prec X_{j}^{\mathrm{t}} \backslash\left\{y_{i}^{1}\right\}
$$

holds and implies $X_{j}^{\mathrm{t}} \backslash\left\{y_{i}^{1}\right\} \prec X_{j}^{\mathrm{t}} \backslash\left\{y_{i}^{1}\right\}$ by transitivity. If a variable, say $V_{j}$, occurs negatively in $C_{i}$, we switch $X_{j}^{\mathrm{t}}$ and $X_{j}^{\mathrm{f}}$ and enforce $X_{j}^{\mathrm{t}} \backslash\left\{y_{i}^{1}\right\} \prec X_{k}^{\mathrm{t}} \backslash\left\{y_{i}^{2}\right\}$ and $X_{l}^{\mathrm{f}} \backslash\left\{y_{i}^{3}\right\} \prec X_{j}^{\mathrm{f}} \backslash\left\{y_{i}^{1}\right\}$. 
Next, we show how we can enforce these preferences. Assume we want to enforce $X_{j}^{a} \backslash\left\{y_{i}^{1}\right\} \prec X_{k}^{b} \backslash\left\{y_{i}^{2}\right\}$ for $a, b \in\{\mathrm{t}, \mathrm{f}\}$. We add

$$
\left\{z_{i}^{1}\right\},\left\{z_{i}^{1}, \max _{i}^{1}\right\} \text { and }\left(X_{j}^{a} \backslash\left\{y_{i}^{1}\right\}\right) \cup\left\{\max _{i}^{1}\right\}
$$

Our goal is to enforce $\left(X_{j}^{a} \backslash\left\{y_{i}^{1}\right\}\right) \cup\left\{\max _{i}^{1}\right\} \prec\left\{z_{i}^{1}, \max _{i}^{1}\right\}$ which forces by reverse strict independence $X_{j}^{a} \backslash\left\{y_{i}^{1}\right\} \prec\left\{z_{i}^{1}\right\}$. Then, we enforce $\left\{z_{i}^{1}\right\} \prec X_{k}^{b} \backslash\left\{y_{i}^{2}\right\}$ to get by transitivity $X_{j}^{a} \backslash\left\{y_{i}^{1}\right\} \prec X_{k}^{b} \backslash\left\{y_{i}^{2}\right\}$ as desired. To enforce

$$
\left(X_{j}^{a} \backslash\left\{y_{i}^{1}\right\}\right) \cup\left\{\max _{i}^{1}\right\} \prec\left\{z_{i}^{1}, \max _{i}^{1}\right\}
$$

we add a sequence of sets $A_{1}, A_{2}, \ldots, A_{l}$ such that

- $A_{1}=\left(X_{j}^{a} \backslash\left\{y_{i}^{1}, z_{i}^{1}\right)\right\} \cup\left\{\max _{i}^{1}\right\}$,

- $A_{i+1}=A_{i} \backslash\left\{\min _{\leq}\left(A_{i}\right)\right\}$

- and $A_{l}=\left\{\max _{i}^{1}\right\}$.

This enforces by dominance $A_{1} \prec A_{2} \prec \cdots \prec A_{l}$ which enforces by transitivity

$$
A_{1}=\left(X_{j}^{a} \backslash\left\{y_{i}^{1}, z_{i}^{1}\right\}\right) \cup\left\{\max _{i}^{1}\right\} \prec\left\{\max _{i}^{1}\right\}=A_{l} .
$$

Finally, as desired, it follows from strict independence that $\left(X_{j}^{a} \backslash\left\{y_{i}^{1}\right\}\right) \cup\left\{\max _{i}^{1}\right\} \prec$ $\left\{z_{i}, \max _{i}^{1}\right\}$. Using the same idea and $\min _{i}^{1}$ we enforce $\left\{z_{i}^{1}\right\} \prec X_{k}^{b} \backslash\left\{y_{i}^{2}\right\}$ finishing the construction for $X_{j}^{a} \backslash\left\{y_{i}^{1}\right\} \prec X_{k}^{b} \backslash\left\{y_{i}^{2}\right\}$. We enforce the other preferences for that clause, i.e., $X_{k}^{c} \backslash\left\{y_{i}^{2}\right\} \prec X_{l}^{d} \backslash\left\{y_{i}^{3}\right\}$ and $X_{l}^{e} \backslash\left\{y_{i}^{3}\right\} \prec X_{j}^{a} \backslash\left\{y_{i}^{1}\right\}$ for $c, d, e \in\{\mathrm{t}, \mathrm{f}\}$, similarly using $z_{i}^{2}, \max _{i}^{2}$ and $\min _{i}^{2}$ resp. $z_{i}^{3}, \max _{i}^{3}$ and $\min _{i}^{3}$. We repeat this procedure for every clause. We call the sets added in this step the Class 3 sets and write $C l_{3}$ for the collection of all Class 3 sets. Furthermore, we write $\mathrm{Cl}_{3}^{+}$for the Class 3 sets that contain an element $\max _{i}^{a}$ for some $i$ and $a$. Similarly, we write $C l_{3}^{-}$for the Class 3 sets that contain an element $\min _{i}^{a}$ for some $i$ and $a$. Finally, we write $C l_{3}^{0}$ for all other sets in Class 3 (which are all of the form $\left.\left\{z_{i}^{a}\right\}\right)$. Now, by construction, $\mathcal{X}$ can only be $D I^{S}$-orderable with respect to $\leq$ if $\phi$ is a positive instance of SAT.

Next, we pick an arbitrary linear order $\leq^{\prime}$ on $X$. We distinguish two cases $v_{1}<^{\prime} v_{2}$ and $v_{2}<^{\prime} v_{1}$. By Lemma 8 it suffices to show that a linear satisfying dominance and strict independence exists in the first case, because $v_{2}<^{\prime} v_{1}$ implies $v_{1}<^{\prime-1} v_{2}$. Hence, we can assume in the following w.l.o.g. $v_{1}<^{\prime} v_{2}$. Now, we want to construct a linear order $\preceq$ on $\mathcal{X}$ that satisfies dominance and strict independence with respect to $\leq^{\prime}$ if $\phi$ is satisfiable. For the readers convenience, we summarize which sets are contained in $\mathcal{X}$ and hence must be taken into account when constructing $\preceq$ : 
Class 1: $X_{i}^{\mathrm{t}}=Y \cup\left\{x_{i, 1}^{-}, x_{i, 1}^{+}\right\}$and $X_{i}^{\mathrm{f}}=Y \cup\left\{x_{i, 2}^{-}, x_{i, 2}^{+}\right\}$for every variable $V_{i}$ of $\phi$.

Class 2: $X_{j}^{\mathrm{t}} \backslash\left\{y_{i}^{a}\right\}$ and $X_{j}^{\mathrm{f}} \backslash\left\{y_{i}^{a}\right\}$, where $a \in\{1,2,3\}$ and $i$ and $j$ are such that $V_{j}$ is in clause $C_{i}$.

Class 3: For each $i \leq m$ and $a \in\{1,2,3\}$ and for some $b, c \in\{\mathrm{t}, \mathrm{f}\}, d \in\{1,2,3\}$ and $j, k$ such that $V_{j}$ and $V_{k}$ are variables in clause $C_{i}$ :

- $\left\{z_{i}^{a}\right\},\left\{z_{i}^{a}, \max _{i}^{a}\right\},\left\{z_{i}^{a}, \min _{i}^{a}\right\},\left(X_{j}^{b} \backslash\left\{y_{i}^{a}\right\}\right) \cup\left\{\max _{i}^{a}\right\},\left(X_{k}^{c} \backslash\left\{y_{i}^{d}\right\}\right) \cup\left\{\min _{i}^{a}\right\}$,

- $A_{1}=\left(X_{j}^{b} \backslash\left\{y_{i}^{a}, z_{i}^{a}\right\}\right) \cup\left\{\max _{i}^{a}\right\}, \ldots, A_{l+1}=A_{l} \backslash\left\{\min _{\leq}\left(A_{l}\right)\right\}, \ldots, A_{o}=\left\{\max _{i}^{a}\right\}$,

- $B_{1}=\left(X_{k}^{c} \backslash\left\{y_{i}^{d}, z_{i}^{a}\right\}\right) \cup\left\{\min _{i}^{a}\right\}, \ldots, B_{l+1}=B_{l} \backslash\left\{\max _{\leq}\left(B_{l}\right)\right\}, \ldots, B_{p}=\left\{\min _{i}^{a}\right\}$.

Now, we construct the order $\preceq$ on $\mathcal{X}$ in several steps. First, we construct from a satisfying assignment of $\phi$ an order on $C l_{1} \cup \mathrm{Cl}_{2}$.

Ordering the sets in $C l_{1} \cup C l_{2}$ : Intuitively, we just order all pairs $\left(X_{i}^{\mathrm{f}}, X_{i}^{\mathrm{t}}\right)$ according to the satisfying assignment of $\phi$ and then 'project' this order down on the Class 2 sets, i.e., we order $X_{j}^{\mathrm{f}} \backslash\left\{y_{i}^{a}\right\}$ and $X_{j}^{\mathrm{t}} \backslash\left\{y_{i}^{a}\right\}$ the same way as $\left(X_{i}^{\mathrm{f}}\right.$ and $\left.X_{i}^{\mathrm{t}}\right)$. Finally, we want to extend this partial order to a linear order on $C l_{1} \cup C l_{2}$. In doing so, we only have to pay attention to the two possible applications of dominance, namely if $y_{i}^{a}$ is either the minimal or maximal element of $X_{i}^{\mathrm{f}}$ resp. $X_{i}^{\mathrm{t}}$. (All other applications of dominance and independence are already covered by the order implied by the assignment and its projection.) Formally, we construct the order as follows.

We start by ordering the sets $X_{i}^{\mathrm{f}}$ and $X_{i}^{\mathrm{t}}$ according to the satisfying assignment of $\phi$, i.e., $X_{i}^{\mathrm{t}} \prec X_{i}^{\mathrm{f}}$ if $V_{i}$ is false in the assignment and $X_{i}^{\mathrm{f}} \prec X_{i}^{\mathrm{t}}$ if it is true. Then, we project this order down to the Class 2 sets by reverse strict independence. Furthermore, we add the preferences that we enforced in the previous section. Finally, we take the transitive closure of this order. It is clear by construction that this is an acyclic partial order if and only if $\phi$ is satisfiable. Now, for any clause $C_{i}$, we fix any linear order on the sets

$$
X_{j}^{\mathrm{f}} \backslash\left\{y_{i}^{1}\right\}, X_{j}^{\mathrm{t}} \backslash\left\{y_{i}^{1}\right\}, X_{k}^{\mathrm{f}} \backslash\left\{y_{i}^{2}\right\}, X_{k}^{\mathrm{t}} \backslash\left\{y_{i}^{2}\right\}, X_{l}^{\mathrm{f}} \backslash\left\{y_{i}^{3}\right\} \text { and } X_{l}^{\mathrm{t}} \backslash\left\{y_{i}^{3}\right\}
$$

that extends this order.

For the Class 1 sets we have ordered all pairs $\left(X_{i}^{\mathrm{f}}, X_{i}^{\mathrm{t}}\right)$ but we still have to fix an order between these pairs. For the Class 2 sets, we have fixed an order on between all sets introduced for a single clause, but we have to fix an order between sets from different clauses. Now, we observe that $A, A \cup\{x\} \in C l_{1} \cup C l_{2}$ implies that $A \in C l_{1}, A \cup\{x\} \in C l_{2}$ and $x=y_{i}^{a}$ for $j \leq m$ and $a \leq 3$ as all Class 1 sets differ from all other Class 1 sets in at least two elements and all Class 2 sets differ from all other Class 2 sets in at least two elements. Hence the only possible application of strict independence on Class 1 and 2 is the one already covered by construction. Dominance is applicable only if $y_{i}^{a}$ for some $i$ and $a$ is the minimal or maximal element of the set it gets removed from. Observe that $y_{i}^{a}$ can only be the maximal element of the set it is removed from if $y_{j}^{b}<y_{i}^{a}$ holds for all $j \neq i$ and $b \neq a$. Hence, there can only be one case where $y_{i}^{a}$ is the maximal element of the set it is removed from and, by a similar argument, one instance where it is the minimal element. Therefore, there are at most two possible applications of dominance. We fix two orders, 
one on the pairs that will be used to order the Class 1 sets and one on the clauses that will be used to order the Class 2 sets. We have to make sure that these orders are compatible with these applications of dominance.

First, assume the minimal element $y_{i^{-}}^{a^{-}}$of the form $y_{i}^{a}$ and the maximal element $y_{i^{+}}^{a^{+}}$of the form $y_{i}^{a}$ are used for the same clause. Let $X_{j}^{b}$ and $X_{k}^{c}$ be the sets such that $X_{j}^{b} \backslash\left\{y_{i^{-}}^{a^{-}}\right\} \in \mathcal{X}$ and $X_{k}^{c} \backslash\left\{y_{i^{+}}^{a^{+}}\right\} \in \mathcal{X}$ holds. Then, by construction $j \neq k$, as, for a given clause, we remove the same element $y_{i}^{a}$ from both $X_{j}^{\mathrm{t}}$ and $X_{j}^{\mathrm{f}}$. In that case we fix any linear order $\leq$ " on the pairs $\left(X_{i}^{\mathrm{f}}, X_{i}^{\mathrm{t}}\right)$ such that $\left(X_{j}^{\mathrm{f}}, X_{j}^{\mathrm{t}}\right) \leq^{\prime \prime}\left(X_{k}^{\mathrm{f}}, X_{k}^{\mathrm{t}}\right)$ holds and an arbitrary order on the clauses. Then, we set $X_{i}^{\mathrm{f}} \prec A$ and $X_{i}^{\mathrm{t}} \prec A$ for every $A \in C l_{2}$ if $\left(X_{i}^{\mathrm{f}}, X_{i}^{\mathrm{t}}\right)<^{\prime \prime}\left(X_{j}^{\mathrm{f}}, X_{j}^{\mathrm{t}}\right)$. Furthermore, we set $A \prec X_{i}^{\mathrm{f}}$ and $A \prec X_{i}^{\mathrm{t}}$ for every $A \in C l_{2}$ if $\left(X_{j}^{\mathrm{f}}, X_{j}^{\mathrm{t}}\right)<{ }^{\prime \prime}\left(X_{i}^{\mathrm{f}}, X_{i}^{\mathrm{t}}\right)$. This is obviously a linear order and we have $X_{j}^{b} \prec X_{j}^{b} \backslash\left\{y_{i^{-}}^{a^{-}}\right\}$and $X_{k}^{c} \backslash\left\{y_{i^{+}}^{a^{+}}\right\} \prec X_{k}^{c}$ for $b, c \leq 2$. Hence the constructed order on $C l_{1} \cup C l_{2}$ satisfies dominance.

Now, assume the minimal element $y_{i^{-}}^{a^{-}}$of the form $y_{i}^{a}$ and the maximal element $y_{i^{+}}^{a^{+}}$of the form $y_{i}^{a}$ are used for different clauses $C_{i^{-}}$and $C_{i^{+}}$. We fix any order $\leq^{\prime \prime}$ on the clauses such that $C_{i^{+}}$is smaller than $C_{i^{-}}$and an arbitrary order on the pairs. Additionally, we set $A \prec B$ for all $A \in C l_{2}$ and $B \in C l_{1}$ if $A$ was introduced for a clause that is smaller equal $C_{i^{+}}$with respect to $\leq^{\prime \prime}$. Furthermore, we set $B \prec A$ for all $A \in C l_{2}$ and $B \in C l_{1}$ if $A$ was introduce for a clause that is larger than $C_{i^{+}}$with respect to $\leq^{\prime \prime}$. This is obviously a linear order and we have $X_{j}^{b} \prec X_{j}^{b} \backslash\left\{y_{i^{-}}^{a^{-}}\right\}$and $X_{k}^{c} \backslash\left\{y_{i^{+}}^{a^{+}}\right\} \prec X_{k}^{c}$ for $b, c \leq 2$. Hence the constructed order on $C l_{1} \cup C l_{2}$ satisfies dominance.

Ordering sets in $C l_{3}$ with same extremum element and $\left\{z_{i}^{a}\right\}$ : We recall that we call the elements $\max _{j}^{a}$ and $\min _{j}^{a}$ extremum elements. We will first define an order between the Class 3 sets that have the same extremum element and $\left\{z_{i}^{a}\right\}$, i.e., that have been introduced to force a same preference. In the following, we write for a set $A$ that contains an extremumelement $m m$ (which is by construction unique) $A_{S}:=\left\{x \in A \mid x<^{\prime} m m\right\}$ for the set of elements in $A$ that are smaller than $m m$ and $A_{L}:=\left\{x \in A \mid m m<^{\prime} x\right\}$ for the set of elements in $A$ that are larger than $\mathrm{mm}$.

We set $A \prec B$ for sets $A, B$ that both contain the same extremum element of the form $\max _{i}^{c}$ if:

- $\max _{\leq^{\prime}}\left(A_{L} \triangle B_{L}\right) \in B$,

- $A_{L}=B_{L}$ and $\min _{\leq^{\prime}}\left(A_{S} \triangle B_{S}\right) \in A$.

Here, $\triangle$ is the symmetric difference operator, i.e., $A \triangle B:=(A \cup B) \backslash(A \cap B)$. Intuitively, this order is a 'nesting' of lexicographic orders. We first check whether $A_{L}$ is smaller than $B_{L}$ according to the leximax order and only if $A_{L}$ and $B_{L}$ are equal we compare $A_{S}$ and $B_{S}$ according to the leximin order. We claim that this order satisfies dominance and strict independence. It satisfies strict independence because for all sets $S, T$ by definition $S \cup\{x\} \triangle T \cup\{x\}=S \triangle T$ for any $x \notin S \cup T$. For dominance, assume $x<<^{\prime} \min _{<^{\prime}}(A)$ and $\max _{i}^{c} \in A, A \cup\{x\}$. Then, $A_{L}=(A \cup\{x\})_{L}$ and $\min _{\leq^{\prime}}\left(A_{S} \triangle(A \cup\{x\})_{S}\right)=x$. Hence, $A \cup\{x\} \prec A$. The case $\max _{<^{\prime}}(A)<^{\prime} x$ is similar.

Next, we add $\left\{z_{i}^{c}\right\}$ to the order. We observe that we have either $X_{j}^{a} \backslash\left\{y_{i}^{b}\right\} \cup\left\{\max _{i}^{c}\right\} \prec$ $\left\{z_{i}^{c}, \max _{i}^{c}\right\}$ or $\left\{z_{i}^{c}, \max _{i}^{c}\right\} \prec X_{j}^{a} \backslash\left\{y_{i}^{b}\right\} \cup\left\{\max _{i}^{c}\right\}$. In the first case, we add $\left\{z_{i}^{c}\right\}$ in the order 
exactly after $X_{j}^{a} \backslash\left\{y_{i}^{b}\right\}$, i.e., we set $\left\{z_{i}^{c}\right\} \prec A$ if $X_{j}^{a} \backslash\left\{y_{i}^{b}\right\} \prec A$ and $A \prec\left\{z_{i}^{c}\right\}$ if $A \preceq X_{j}^{a} \backslash\left\{y_{i}^{b}\right\}$ for all $A \in \mathcal{X} \backslash\left\{z_{i}^{c}\right\}$. In the second case we set $\left\{z_{i}^{c}\right\}$ exactly before $X_{j}^{a} \backslash\left\{y_{i}^{c}\right\}$.

Now, let $X_{k}^{d} \backslash\left\{y_{i}^{e}\right\}$ be the set for which we enforce the preference $X_{j}^{a} \backslash\left\{y_{i}^{c}\right\} \prec X_{k}^{d} \backslash\left\{y_{i}^{e}\right\}$. Then, this implies $\left\{z_{i}^{c}\right\} \prec X_{k}^{d} \backslash\left\{y_{i}^{e}\right\}$, because $X_{j}^{a} \backslash\left\{y_{i}^{b}\right\} \prec X_{k}^{d} \backslash\left\{y_{i}^{e}\right\}$ holds by construction and we added $\left\{z_{i}^{c}\right\}$ just before or just after $X_{j}^{a} \backslash\left\{y_{i}^{b}\right\}$. Therefore, we have to make sure that $\left\{z_{i}^{c}, \min _{i}^{c}\right\} \prec X_{k}^{d} \backslash\left\{y_{i}^{e}\right\} \cup\left\{\min _{i}^{c}\right\}$ holds as intended by the construction to avoid a contradiction. For this we use the fact that $v_{1}<^{\prime} v_{2}$ holds. We set $A \prec B$ for elements $A, B$ if they both contain an element of the form $\min _{i}^{c}$ if:

- $v_{2} \in B$ and $v_{2} \notin A$,

- $v_{2} \in A, B$ or $v_{2} \notin A, B$ and $\max _{\leq^{\prime}}\left(A_{L} \triangle B_{L}\right) \in B$,

- $v_{2} \in A, B$ or $v_{2} \notin A, B, A_{L}=B_{L}$ and $\min _{\leq^{\prime}}\left(A_{S} \triangle B_{S}\right) \in A$.

It is clear that $(\star)$ implies $\left\{z_{i}^{c}, \min _{i}^{c}\right\} \prec X_{k}^{d} \backslash\left\{y_{i}^{e}\right\} \cup\left\{\min _{i}^{c}\right\}$. It is also clear that it satisfies strict independence because the $(\star)$ implies a preference between sets $A \cup\{x\}$ and $B \cup\{x\}$ for $x \notin A \cup B$ iff it implies the same preference for $A$ and $B$. If $(\star)$ is not applicable, strict independence is satisfied by the same argument as above. Now, for dominance $v_{2} \in$ $(A \triangle(A \cup\{x\}))$ implies $x=v_{2}$. Then, $x<\min _{<^{\prime}}(A)$ is not possible because by construction $v_{1} \in A$ holds and we assume $v_{1}<^{\prime} v_{2}$. If we have $\max _{<^{\prime}}(A)<^{\prime} x$ then dominance is satisfied because $A \prec A \cup\{x\}$ holds by $(\star)$. If $x \neq v_{2}$, then $(\star)$ is not applicable and dominance is satisfied by the same argument as above.

Interaction between $C l_{1} \cup C l_{2}$ and $C l_{3}$ : The interaction between the Classes $C l_{1} \cup$ $\mathrm{Cl}_{2}$ and $\mathrm{Cl}_{3}$ can be handled quite straightforwardly, as the only possible application of dominance and strict independence are the ones intended by the construction, i.e., when adding a extremum element to force a preference.

First, we observe that there is no set $A \in C l_{3}$ such that $A \cup\{x\} \in C l_{1} \cup C l_{2}$ holds, as every set in $C_{3}$ either contains an extremum-element or it is a singleton and no set in Class 1 and 2 contains an extremum-element and every set in Class 1 and 2 has more than three elements.

Furthermore, if $A \in C l_{1} \cup C l_{2}$ and $A \cup\{x\} \in C l_{3}$, then $A$ must be of the form $X_{i}^{a} \backslash\left\{y_{j}^{b}\right\}$ and $x$ must be $\max _{j}^{c}$ or $\min _{j}^{d}$. Additionally, by construction, for every $A \in C l_{1} \cup C l_{2}$ there is at most one $x$ such that $A \cup\{x\}$ holds. Therefore, the only possible application of dominance involving sets from $C l_{1} \cup C l_{2}$ and $C l_{3}$ is adding an extremum element $\max _{j}^{c}$ or $\min _{j}^{d}$ to a set $A$ in $C l_{1} \cup C l_{2}$ such that the extremum element is smaller (larger) than all elements in $A$. We have to add preferences that satisfy this application of dominance. Consider $A \in C l_{1} \cup C l_{2}$ and $B \in C l_{3}^{+} \cup C l_{3}^{-}$and let $m m_{B}$ be the unique extremum element in $B$. Furthermore, let $i \leq$ and $a \in\{1,2,3\}$ be the unique values for which $\left\{z_{i}^{a}, m m_{B}\right\}$ is in $\mathcal{X}$. Then, we extend $\preceq$ by

- $A \prec B$ if $z_{i}^{a}<m m_{B}$,

- $B \prec A$ if $m m_{B}<z_{i}^{a}$.

Clearly, the resulting order is still a partial order and it satisfies all possible applications of dominance involving sets from $C l_{1} \cup C l_{2}$ and $C l_{3}$, because $z_{i}^{a} \in A$ for all $i \leq m, a \in\{1,2,3\}$ 
and $A \in C l_{1} \cup C l_{2}$. Furthermore, observe that we do not add any new preferences between sets in $C l_{1} \cup C l_{2}$, but we do add, by transitivity, new comparisons between sets in $\mathrm{Cl}_{3}$ that contain different extremum elements.

Now, for strict independence, the only application with sets from Class 3 and sets not from Class 3 is lifting a preference between a set $A$ and $\left\{z_{i}^{a}\right\}$ to a preference between $A \cup\{m m\}$ and $\left\{z_{i}^{a}, m m\right\}$ for some specific extremum element $m m$. By construction $A$ must be of the form $X_{k}^{b} \backslash\left\{y_{i}^{a}\right\}$ and hence strict independence is satisfied as we have seen in the paragraph above.

Ordering sets in $\mathrm{Cl}_{3}$ with different (or no) extremum elements: Finally, we have to extend the order $\preceq$ to the whole Class 3 . First, observe that two sets with different extremum elements differ in at least two elements. Hence, there is no possible application of dominance with sets containing different extremum elements. The only possible application of strict independence that is not already satisfied by $\preceq$ is adding the same element to two sets containing different extremum elements. In order to make sure that these applications of strict independence are satisfied, we order the elements with different extremum element with an order that is only based on what extremum elements they contain. We fix an arbitrary linear order $\leq_{m m}$ on the extremum elements that is compatible with the preferences introduced in the paragraph above, i.e., if $\left\{m m_{1}, z_{i}^{a}\right\},\left\{m m_{2}, z_{j}^{b}\right\} \in \mathcal{X}, m m_{1}<z_{i}^{a}$ and $z_{j}^{b}<m m$ hold then $m m_{1} \leq_{m m} m m_{2}$ must hold. We extend $\preceq$ by adding for all sets $A$ and $B$ that contain different extremum elements $m m_{A}$ and $m m_{B}$ respectively

- $A \prec B$ if $m m_{A}<_{m m} m m_{B}$,

- $B \prec A$ if $m m_{B}<_{m m} m m_{A}$.

Clearly, this is compatible with the preference between sets with different extremum elements added in the paragraph above. Furthermore, we know for all $A, B$ that contain an extremum element that for all $x \in X$ such that $A \cup\{x\}, B \cup\{x\} \in \mathcal{X}$ holds that $A \cup\{x\}$ contains the same unique extremum element as $A$ and $B \cup\{x\}$ contains the same unique extremum element as $B$. Hence, if $A$ and $B$ are Class 3 elements that contain different extremum elements, then $A \prec B$ implies $A \cup\{x\} \prec B \cup\{x\}$.

Furthermore, we observe that any Class 3 set that does not contain an extremum element must be of the form $\left\{z_{i}^{a}\right\}$ for some $z_{i}^{a} \in X$. Now, if $\left\{z_{i}^{a}\right\} \cup\{x\}$ is in $\mathcal{X}$ then $x$ must be either $\min _{i}^{a}$ or $\max _{i}^{a}$. Furthermore, there is no other Class 3 set $A$ such that $A \cup\{x\}$ is in $\mathcal{X}$. Therefore, no application of strict independence involving $\left\{z_{i}^{a}\right\}$ is possible. Finally, as $\left\{z_{i}^{a}\right\}$ is placed next to a Class 2 set $X_{j}^{b} \backslash\left\{y_{i}^{c}\right\},\left\{m m, z_{i}^{a}\right\} \in \mathcal{X}$ and $m m<z_{i}^{a}$ implies $\left\{m m, z_{i}^{a}\right\} \prec\left\{z_{i}^{a}\right\}$ by the construction in the paragraph above. Similarly, $\left\{m m, z_{i}^{a}\right\} \in \mathcal{X}$ and $z_{i}^{a}<m m$ implies $\left\{z_{i}^{a}\right\} \prec\left\{z_{i}^{a}, m m\right\}$. Therefore, any application of dominance involving $\left\{z_{i}^{a}\right\}$ is satisfied.

Completion of $\preceq$ : Finally, because $\preceq$ is a partial order there is a linear order that is a completion of $\preceq$. As we have seen above, $\preceq$ satisfies all possible applications of strict independence and dominance. Thus, any completion of $\preceq$ satisfies dominance and strict independence.

We have shown that there is a linear order on $\mathcal{X}$ that satisfies dominance and strict independence with respect to a fixed order $\leq$ if and only if $\phi$ is satisfiable. Furthermore, 
we have shown how to use a satisfying assignment of $\phi$ to construct for any linear order $\leq^{\prime}$ on $X$ a linear order $\preceq$ on $\mathcal{X}$ that satisfies dominance and strict independence. This construction clearly also works for the fixed order $\leq$. In other words $\phi$ is satisfiable if and only if $(X, \mathcal{X}, \leq)$ is a positive instance of $D I^{S}$-LO-Orderability. Hence, the reduction above also shows that $D I^{S}$-LO-ORDERABILITY is NP-complete.

Corollary 22. $D I^{S}$-LO-ORDERABILITY is NP-complete.

Proof. It follows from the reduction above that $D I^{S}$-LO-ORDERABILITY is NP-hard. Furthermore, it is clear that $D I^{S}$-LO-ORDERABILITY is in NP: We can guess a linear order $\preceq$ on $\mathcal{X}$ and then check in polynomial time if $\preceq$ satisfies dominance and strict independence with respect to $\leq$.

This result was already shown by Maly and Woltran (2017). However, the new reduction presented here will be useful to prove further results. A variation of this reduction shows that we can additionally add extension to the required axioms without changing the complexity of the problem. Essentially, we modify the family $\mathcal{X}$ such that it does not contain any singletons. This can be achieved by replacing singletons with two element sets.

Corollary 23. Strong $D I^{S} E$-LO-ORDERABILITY is NP-hard. $D I^{S}$ E-LO-Orderability is NP-complete.

Proof. First, observe that $D I^{S} E$-LO-Orderability is in NP for the same reason that $D I^{S}$-LO-OrderabiLity is, because it can also be checked in polynomial time if a linear order satisfies the extension rule.

To show that Strong $D I^{S} E$-LO-Orderability and $D I^{S} E$-LO-Orderability are NP-hard we have to modify the reduction above slightly. In general, the order on the singletons does not satisfy the extension rule. One way to solve this problem is replacing all singletons by two element sets. All singletons that appear in the reduction are of the form $\left\{z_{k}^{a}\right\},\left\{\min _{i}^{b}\right\}$ or $\left\{\max _{j}^{c}\right\}$ for some $z_{k}^{a}, \min _{i}^{b}, \max _{j}^{c} \in X$. For all $z_{i}^{a} \in X$, replace in the reduction above every mentioning of $z_{i}^{a}$ by $z_{i}^{a, 1}, z_{i}^{a, 2}$ and similarly replace for all $\min _{i}^{b}$, $\max _{j}^{c} \in$ $X$ every mentioning of $\min _{i}^{b}$ and $\max _{j}^{c}$ by $\overline{\min _{i}^{b}}, \min _{i}^{b}$ and $\overline{\max _{i}^{c}}, \max _{i}^{c}$, respectively. In the critical linear order, we replace $z_{i}^{a}$ by $z_{i}^{a, 1}<z_{i}^{a, 2}, \min _{i}^{b}$ by $\overline{\min _{i}^{b, *}}<\min _{i}^{b}$ and $\max _{j}^{c}$ by $\max _{i}^{c}<\overline{\max _{i}^{c}}$. Finally, we add the additional set $\left(X_{j}^{a} \backslash\left\{y_{i}^{1}, z_{i}^{a, 1}\right\}\right) \cup\left\{\max _{i}^{1}, \overline{\max _{i}^{1}}\right\}$, $\left\{z_{i}^{a, 2}, \max _{i}^{1}, \overline{\max _{i}^{1}}\right\},\left(X_{j}^{a} \backslash\left\{y_{i}^{1}\right\}\right) \cup\left\{\max _{i}^{1}\right\}$ and $\left\{z_{i}^{a, 1}, z_{i}^{a, 2}, \max _{i}^{1}\right\}$.

Then

$$
\left(X_{j}^{a} \backslash\left\{y_{i}^{1}, z_{i}^{a, 1}, z_{i}^{a, 2}\right\}\right) \cup\left\{\max _{i}^{1}, \overline{\max _{i}^{1}}\right\} \prec\left\{\max _{i}^{1}, \overline{\max _{i}^{1}}\right\}
$$

implies by two applications of strict independence

$$
\left(X_{j}^{a} \backslash\left\{y_{i}^{1}\right\}\right) \cup\left\{\max _{i}^{1}, \overline{\max _{i}^{1}}\right\} \prec\left\{z_{i}^{a, 1}, z_{i}^{a, 2}, \max _{i}^{1}, \overline{\max _{i}^{1}}\right\} .
$$

This, in turn, implies by two applications of reverse strict independence

$$
\left(X_{j}^{a} \backslash\left\{y_{i}^{1}\right\}\right) \prec\left\{z_{i}^{a, 1}, z_{i}^{a, 2}\right\} .
$$


When constructing a linear order $\preceq$ based on a satisfying assignment, we can treat the four new sets $\left(X_{j}^{a} \backslash\left\{y_{i}^{1}, z_{i}^{a, 1}\right\}\right) \cup\left\{\max _{i}^{1}, \overline{\max _{i}^{1}}\right\},\left\{z_{i}^{a, 2}, \max _{i}^{1}, \overline{\max _{i}^{1}}\right\},\left(X_{j}^{a} \backslash\left\{y_{i}^{1}\right\}\right) \cup\left\{\max _{i}^{1}\right\}$ and $\left\{z_{i}^{a, 1}, z_{i}^{a, 2}, \max _{i}^{1}\right\}$ the same as any other set containing $\max _{i}^{1}$. It can be checked that the rest of the construction works as before. Now, $\mathcal{X}$ does not contain any singletons. Therefore, the linear order constructed in the second part of the reduction satisfies the extension rule vacuously.

\section{$D I^{S}$-WO-ORDERABILITY}

Now, we relax the requirement that the lifted order needs to be antisymmetric. As it turns out, this does not change the complexity of deciding whether dominance and strict independence are compatible. To show this, we need to modify the reduction given above. Again, we first state the result for strong orderability, even though we will improve that result in the next section (Theorem 27). The NP-completeness of the $\leq$-orderability version will follow directly.

Proposition 24. Strong $D I^{S}$-WO-Orderability is NP-hard.

Proof. We will modify the reduction used for Proposition 21. We have to compensate for the fact that the lifted order $\preceq$ does not need to be antisymmetric. First, we have to make sure that all preferences between sets $X_{i}^{\mathrm{f}}$ and $X_{i}^{\mathrm{t}}$ are strict. Otherwise, $\preceq$ would not encode a valid truth assignment. We can add elements and sets that lead to a contradiction if the preference is not strict. This can be done as follows: Assume we want to enforce that the preferences between two sets $X_{i}^{\mathrm{f}}$ and $X_{i}^{\mathrm{t}}$ is strict. Then we add sets $A, B, C$ and $D$ and enforce preferences such that $X_{i}^{\mathrm{f}} \preceq X_{i}^{\mathrm{t}}$ implies $A \prec B$ by transitivity and $X_{i}^{\mathrm{t}} \preceq X_{i}^{\mathrm{f}}$ implies $D \prec C$ by transitivity. Furthermore, we add sets such that $A \prec B$ and $D \prec C$ lead to a contradiction if they hold at the same time. Then, $X_{i}^{\mathrm{f}} \preceq X_{i}^{\mathrm{t}}$ and $X_{i}^{\mathrm{t}} \preceq X_{i}^{\mathrm{f}}$ cannot hold at the same time. Hence, either $X_{i}^{\mathrm{f}} \prec X_{i}^{\mathrm{t}}$ or $X_{i}^{\mathrm{t}} \prec X_{i}^{\mathrm{f}}$ must hold.

Furthermore, if $\preceq$ is not linear, then it does not need to satisfy reverse strict independence even if it satisfies strict independence. Reverse strict independence was only used once on the reduction for Proposition 21, namely to infer that $\left(X_{j}^{a} \backslash\left\{y_{i}^{1}\right\}\right) \cup\left\{\max _{i}^{1}\right\} \prec\left\{z_{i}^{1}, \max _{i}^{1}\right\}$ implies $X_{j}^{a} \backslash\left\{y_{i}^{1}\right\} \prec\left\{z_{i}^{1}\right\}$. Therefore, we need to adapt the way that we enforce preferences to a method that does not require reverse strict independence. This can be done as follows for a desired preference $X_{i}^{a} \backslash\left\{y_{j}^{b}\right\} \prec X_{i}^{c} \backslash\left\{y_{j}^{b}\right\}$ : We replace every element $z_{j}^{b}$ by two elements $z_{j}^{b}$ and $\overline{z_{j}^{b}}$, set $z_{j}^{b}<\overline{z_{j}^{b}}$ and add the sets $\left\{z_{j}^{b}\right\},\left\{z_{j}^{b}, \overline{z_{j}^{b}}\right\},\left\{\overline{z_{j}^{b}}\right\}$ to $\mathcal{X}$. By dominance this enforces $\left\{z_{j}^{b}\right\} \prec\left\{\overline{z_{j}^{b}}\right\}$. Now, using a similar construction as before, we can enforce $X_{i}^{a} \backslash\left\{y_{j}^{b}\right\} \preceq\left\{z_{j}^{b}\right\}$ and $\left\{\overline{z_{j}^{b}}\right\} \preceq X_{k}^{c} \backslash\left\{y_{j}^{b}\right\}$. Then, we have

$$
X_{i}^{a} \backslash\left\{y_{j}^{b}\right\} \preceq\left\{z_{j}^{b}\right\} \prec\left\{\overline{z_{j}^{b}}\right\} \preceq X_{k}^{c} \backslash\left\{y_{j}^{b}\right\}
$$

and hence by transitivity the desired strict preference $X_{i}^{a} \backslash\left\{y_{j}^{b}\right\} \prec X_{i}^{c} \backslash\left\{y_{j}^{b}\right\}$. To enforce $X_{i}^{a} \backslash\left\{y_{j}^{b}\right\} \preceq\left\{z_{j}^{b}\right\}$ we add the same sequence $A_{1}, \ldots, A_{l}$ as in the proof of Proposition 21 . This enforces as before

$$
\left(X_{i}^{a} \backslash\left\{y_{j}^{b}\right\}\right) \cup\left\{\max _{j}^{b}\right\} \prec\left\{z_{j}^{b}, \max _{j}^{b}\right\} .
$$

Now, as $\preceq$ is total, by Proposition 18 it satisfies reverse independence. Therefore, we get the desired $X_{i}^{a} \backslash\left\{y_{j}^{b}\right\} \preceq\left\{z_{j}^{b}\right\}$. We can enforce $\left\{\overline{z_{j}^{b}}\right\} \preceq X_{k}^{c} \backslash\left\{y_{j}^{d}\right\}$ similarly. 

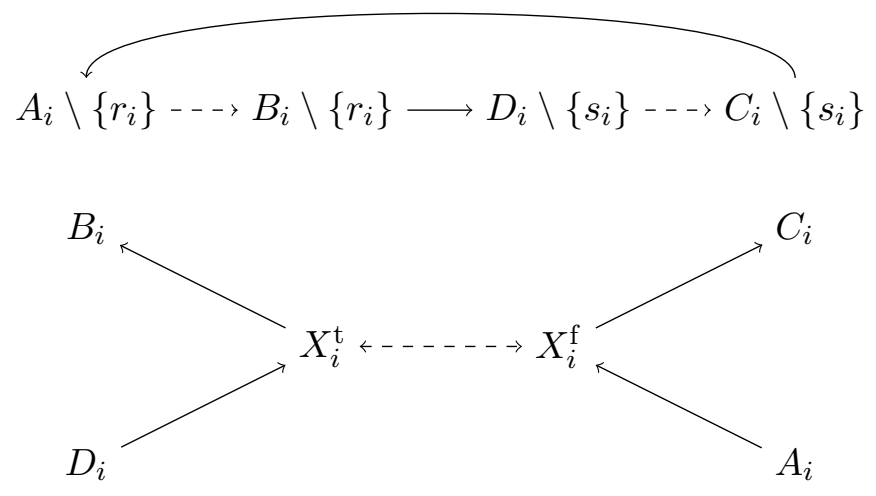

Figure 3: Enforcing strictness.

Next, we want to enforce that all preferences between sets $X_{i}^{\mathrm{f}}$ and $X_{i}^{\mathrm{t}}$ are strict using the idea described at the beginning of the proof. We will add additional sets that lead to cyclic preferences if $X_{i}^{\mathrm{f}} \preceq X_{i}^{\mathrm{t}}$ and $X_{i}^{\mathrm{t}} \preceq X_{i}^{\mathrm{f}}$ hold at the same time. The idea is illustrated in Figure 3. We add for every variable $X_{i}$ new elements

$$
a_{i}^{-}, b_{i}^{-}, c_{i}^{-}, d_{i}^{-}, r_{i}, s_{i}, d_{i}^{+}, c_{i}^{+}, b_{i}^{+}, a_{i}^{+} .
$$

Furthermore, we add them to the critical linear order $\leq$ as follows

$$
\begin{gathered}
\min _{1}^{1}<\min _{1}^{2}<\cdots<\min _{m}^{3}<x_{1,1}^{-}<x_{1,2}^{-}<\cdots<x_{n, 2}^{-}< \\
\mathbf{a}_{\mathbf{i}}^{-}<\mathbf{b}_{\mathbf{i}}^{-}<\mathbf{c}_{\mathbf{i}}^{-}<\mathbf{d}_{\mathbf{i}}^{-}<v_{1}<v_{2}<\mathbf{r}_{\mathbf{i}}<\mathbf{s}_{\mathbf{i}}<\mathbf{d}_{\mathbf{i}}^{+}<\mathbf{c}_{\mathbf{i}}^{+}<\mathbf{b}_{\mathbf{i}}^{+}<\mathbf{a}_{\mathbf{i}}^{+} \\
<z_{1}^{1}<z_{1}^{2} \cdots<z_{m}^{3}<y_{1}^{1}<y_{1}^{2}<\cdots<y_{m}^{3}< \\
x_{1,1}^{+}<x_{1,2}^{+}<\cdots<x_{n, 2}^{+}<\max _{1}^{1}<\max _{1}^{2}<\cdots<\max _{m}^{3}
\end{gathered}
$$

Then, we add new sets

$$
\begin{aligned}
A_{i}:=\left\{a_{i}^{-}, v_{1}, v_{2}, r_{i}, s_{i}, a_{i}^{+}\right\}, B_{i} & :=\left\{b_{i}^{-}, v_{1}, v_{2}, r_{i}, s_{i}, b_{i}^{+}\right\}, \\
C_{i} & :=\left\{c_{i}^{-}, v_{1}, v_{2}, r_{i}, s_{i}, c_{i}^{+}\right\} \text {and } D_{i}:=\left\{d_{i}^{-}, v_{1}, v_{2}, r_{i}, s_{i}, d_{i}^{+}\right\} .
\end{aligned}
$$

Now, let $z_{i}^{a}, \overline{z_{i}^{a}}, \max _{i}^{a}$ and $\min _{i}^{a}$ be new elements where we set $z_{i}^{a}, \overline{z_{i}^{a}} \in Y$. Then, we enforce with the method described above $A_{i} \prec X_{i}^{\mathrm{f}}$ using these new elements. Furthermore, we enforce $X_{i}^{\mathrm{t}} \prec B_{i}, X_{i}^{\mathrm{f}} \prec C_{i}$ and $D_{i} \prec X_{i}^{\mathrm{t}}$. Finally, we add the sets $A_{i} \backslash\left\{r_{i}\right\}, B_{i} \backslash\left\{r_{i}\right\}$, $C_{i} \backslash\left\{s_{i}\right\}$ and $D_{i} \backslash\left\{s_{i}\right\}$ and enforce $B_{i} \backslash\left\{r_{i}\right\} \prec D_{i} \backslash\left\{s_{i}\right\}$ and $C_{i} \backslash\left\{s_{i}\right\} \prec A_{i} \backslash\left\{r_{i}\right\}$. We call the sets added in this step the Class 4 sets. These enforced preference are shown as solid arrows in Figure 3.

Now, we claim that it is not possible for a weak order $\preceq$ to satisfy dominance and strict independence with respect to $\leq$ if $X_{i}^{\mathrm{t}} \sim X_{i}^{\mathrm{f}}$ holds. Assume otherwise that $\preceq$ is a weak order that satisfies dominance and strict independence with respect to $\leq$ such that 
$X_{i}^{\mathrm{t}} \sim X_{i}^{\mathrm{f}}$ holds. Then, $D_{i} \prec X_{i}^{\mathrm{t}} \preceq X_{i}^{\mathrm{f}} \prec C_{i}$ implies $D_{i} \prec C_{i}$ by transitivity and hence $D_{i} \backslash\left\{s_{i}\right\} \preceq C_{i} \backslash\left\{s_{i}\right\}$ by reverse independence. Similarly, $A_{i} \prec X_{i}^{\mathrm{f}} \preceq X_{i}^{\mathrm{t}} \prec B_{i}$ implies $A_{i} \prec B_{i}$ by transitivity and hence $A_{i} \backslash\left\{r_{i}\right\} \preceq B_{i} \backslash\left\{r_{i}\right\}$ by reverse independence. However, this leads to a contradiction by

$$
A_{i} \backslash\left\{r_{i}\right\} \preceq B_{i} \backslash\left\{r_{i}\right\} \prec D_{i} \backslash\left\{s_{i}\right\} \preceq C_{i} \backslash\left\{s_{i}\right\} \prec A_{i} \backslash\left\{r_{i}\right\} .
$$

Now, as before $\mathcal{X}$ can only be $D I^{S}$-orderable with respect to $\leq$ if $\phi$ is a positive instance of SAT.

It remains to show that the modified family $\mathcal{X}$ is strongly $D I^{S}$-orderable if $\phi$ is a positive instance of SAT. As before, let $\leq^{\prime}$ be an arbitrary linear order on $X$. If $\phi$ is a positive instance of SAT, we can construct a weak order on $\mathcal{X}$ that satisfies dominance and strict independence as before, with the following modifications: We replace $z_{i}^{e}$ by the block $\left\{z_{i}^{e}\right\} \prec\left\{z_{i}^{e}, \overline{z_{i}^{e}}\right\} \prec\left\{\overline{z_{i}^{e}}\right\}$ if $z_{i}^{e}<\overline{z_{i}^{e}}$ and by the block $\left\{\overline{z_{i}^{e}}\right\} \prec\left\{z_{i}^{e}, \overline{z_{i}^{e}}\right\} \prec\left\{z_{i}^{e}\right\}$ if $\overline{z_{i}^{e}}<z_{i}^{e}$.

It remains to add the Class 4 sets to the order. The Class 4 sets used to enforce the preferences $A_{i} \prec X_{i}^{\mathrm{f}}, X_{i}^{\mathrm{t}} \prec B_{i}, X_{i}^{\mathrm{f}} \prec C_{i}$ and $D_{i} \prec X_{i}^{\mathrm{t}}$ can be ordered the same way as the Class 3 sets. As before, we use the fact that we can assume $v_{1}<^{\prime} v_{2}$ to ensure that this order is compatible with the enforced preferences. For a specific variable $V_{i}$ we set by construction either $X_{i}^{\mathrm{t}} \prec X_{i}^{\mathrm{f}}$ or $X_{i}^{\mathrm{f}} \prec X_{i}^{\mathrm{t}}$. We assume $X_{i}^{\mathrm{t}} \prec X_{i}^{\mathrm{f}}$. The other case is symmetric. Then, we add $D_{i}$ in $\preceq$ exactly before $X_{i}^{\mathrm{t}}$ and $B_{i}$ exactly after $X_{i}^{\mathrm{t}}$. Similarly, we add $A_{i}$ exactly before $X_{i}^{\mathrm{f}}$ and $C_{i}$ exactly after $X_{i}^{\mathrm{f}}$. Then, we have

$$
D_{i} \prec X_{i}^{\mathrm{t}} \prec B_{i} \prec A_{i} \prec X_{i}^{\mathrm{f}} \prec C_{i}
$$

which is compatible with the forced preferences.

Now, consider the group $A_{i} \backslash\left\{r_{i}\right\}, B_{i} \backslash\left\{r_{i}\right\}, C_{i} \backslash\left\{s_{i}\right\}$ and $D_{i} \backslash\left\{s_{i}\right\}$. We observe that all sets in this group differ in at least two elements. Therefore, we only have to set $B_{i} \backslash\left\{r_{i}\right\} \preceq$ $A_{i} \backslash\left\{r_{i}\right\}$ and $D_{i} \backslash\left\{s_{i}\right\} \preceq C_{i} \backslash\left\{s_{i}\right\}$ in order to satisfies reverse independence. Furthermore, we have to satisfy dominance if $r_{i}$ and/or $s_{i}$ are the largest element of the set they are removed from. This can be satisfied by a straightforward construction that respects the enforced preferences unless $r_{i}$ is the maximal element of $A_{i}$ and $s_{i}$ is the minimal element of $C_{i}$ or alternatively if $r_{i}$ is the maximal element of $B_{i}$ and $s_{i}$ is the minimal element of $D_{i}$. We describe the construction for the first case: We have to set $A_{i} \backslash\left\{r_{i}\right\} \prec A_{i}$ and $C_{i} \prec C_{i} \backslash\left\{s_{i}\right\}$ which implies $A_{i} \backslash\left\{r_{i}\right\} \prec C_{i} \backslash\left\{s_{i}\right\}$ contrary to the preference we wanted to enforce in the construction. We use the fact that $r_{i}$ is the maximal element of $A_{i}$ and $s_{i}$ is the minimal element of $C_{i}$ to define an order that allows this. Let $z_{i}^{r, a}$ and $\max _{i}^{r, a}$ be the new elements used to enforce $\left\{z_{i}^{r, a}\right\} \prec A_{i} \backslash\left\{r_{i}\right\}$. Then, we use the same order as for the other Class 3 sets, i.e., we set $A \preceq B$ for the sets $A, B$ such that $\max _{i}^{r, a} \in A, B$ if

- $v_{2} \in A$ and $v_{2} \notin B$

- $v_{2} \in A, B$ or $v_{2} \notin A, B$ and $\max _{\leq^{\prime}}\left(A_{L} \triangle B_{L}\right) \in B$,

- $v_{2} \in A, B$ or $v_{2} \notin A, B, A_{L}=B_{L}$ and $\min _{\leq^{\prime}}\left(A_{S} \triangle B_{S}\right) \in A$.

where $A_{L}:=\left\{x \in A \mid \max _{i}^{r, a}<^{\prime} x\right\}$ and $A_{S}:=\left\{x \in A \mid x<^{\prime} \max _{i}^{r, a}\right\}$. This order satisfies dominance and strict independence because the element $s_{i}$ is smaller than $v_{2}$ by assumption and removed later in the sequence $A_{1}, \ldots, A_{k}$. Furthermore, this implies $\left(A_{i} \backslash\right.$ 
$\left.\left\{r_{i}\right\}\right) \cup\left\{\max _{i}^{r, a}\right\} \prec\left\{z_{i}^{r, a}, \max _{i}^{r, a}\right\}$, which implies $A_{i} \backslash\left\{r_{i}\right\} \prec\left\{z_{i}^{r, a}\right\}$. This allows us to set $A_{i} \backslash\left\{r_{i}\right\} \prec C_{i} \backslash\left\{s_{i}\right\}$. Then, we can place $A_{i} \backslash\left\{r_{i}\right\}$ just before $A_{i}$ and $C_{i} \backslash\left\{s_{i}\right\}$ just after $C_{i}$ to get an order that satisfies dominance and independence.

As in the case of Strong $D I^{S} E$-LO-Orderability we have shown that there is a weak order on $\mathcal{X}$ that satisfies dominance and strict independence with respect to a fixed order $\leq$ if only if $\phi$ is satisfiable. Therefore, this reduction again also works for $D I^{S}$-WOORDERABILITY.

Theorem 25. $D I^{S}$-WO-ORDERABILITY is NP-complete.

Proof. It follows from the reduction above that $D I^{S}$-WO-ORDERABILITY is NP-hard. Furthermore, it is clear that $D I^{S}$-WO-ORDERABILITY is in NP as we can guess a weak order $\preceq$ on $\mathcal{X}$ and then check in polynomial time if $\preceq$ satisfies dominance and strict independence with respect to $\leq$.

Moreover, we can adapt the reduction to the case that extension is additionally required in a similar way as in the proof of Corollary 23.

\section{Corollary 26. $D I^{S} E$-WO-ORDERABILITY is NP-complete.}

Proof. We can adapt the reduction in a similar way as in the proof of Corollary 23. We need to change two things compared to Corollary 23. First of all, in contrast to reverse strict independence we cannot iterate reverse independence. Therefore, we need to adapt the way that we enforce preferences. We add additionally the sets $\left(X_{i}^{a} \backslash\left\{y_{j}^{b}, x_{i}^{-}\right\}\right) \cup\left\{\max _{j}^{b}\right\}$. and $\left(X_{i}^{a} \backslash\left\{y_{j}^{b}, x_{i}^{-}\right\}\right) \cup\left\{\max _{j}^{b}, \overline{\max _{i}^{b}}\right\}$. We observe that for the sequence $A_{1}, \ldots, A_{l}$ added to enforce the preference $\left(X_{j}^{a} \backslash\left\{y_{i}^{b}\right\}\right) \cup\left\{\max _{i}^{c}\right\} \prec\left\{z_{i}, \max _{i}^{d}\right\}$, we have $l>3$ and that the following preference is enforced by dominance

$$
A_{2}=\left(X_{i}^{a} \backslash\left\{y_{j}^{b}, x_{i}^{-}, z_{i}^{a, 1}, z_{i}^{a, 2}\right\}\right) \cup\left\{\max _{j}^{b}, \overline{\max _{i}^{b}}\right\} \prec\left\{\max _{j}^{b}, \overline{\max _{i}^{b}}\right\}=A_{l}
$$

this enforces by strict independence

$$
\left(X_{i}^{a} \backslash\left\{y_{j}^{b}, x_{i}^{-}\right\}\right) \cup\left\{\max _{j}^{b}, \overline{\max _{i}^{b}}\right\} \prec\left\{z_{i}^{a, 1}, z_{i}^{a, 2}, \max _{j}^{b}, \overline{\max _{i}^{b}}\right\} .
$$

Now, by one application of reverse independence, we have

$$
\left(X_{i}^{a} \backslash\left\{y_{j}^{b}, x_{i}^{-}\right\}\right) \cup\left\{\max _{j}^{b},\right\} \preceq\left\{z_{i}^{a, 1}, z_{i}^{a, 2}, \max _{j}^{b}\right\} .
$$

and hence by dominance

$$
\left(X_{i}^{a} \backslash\left\{y_{j}^{b}\right\}\right) \cup\left\{\max _{j}^{b}\right\} \prec\left\{z_{i}^{a, 1}, z_{i}^{a, 2}, \max _{j}^{b}\right\} .
$$

Then, another application of reverse independence implies

$$
\left(X_{j}^{a} \backslash\left\{y_{i}^{1}\right\}\right) \preceq\left\{z_{i}^{a, 1}, z_{i}^{a, 2}\right\} .
$$


Furthermore, the new singleton in the reduction $\overline{z_{i}^{e}}$ also needs to be dealt with. We replace $z_{i}^{e}$ again by two elements $z_{i}^{e, 1}$ and $z_{i}^{e, 2}$. Similarly we replace $\overline{z_{i}^{e}}$ by $\overline{z_{i}^{e, 1}}$ and $\overline{z_{i}^{e, 2}}$. In the critical linear order we set

$$
z_{i}^{e, 1}<z_{i}^{e, 2}<\overline{z_{i}^{e, 1}}<\overline{z_{i}^{e, 2}}
$$

Then, we use these to enforce as in Corollary 23

$$
X_{i}^{a} \backslash\left\{y_{j}^{b}\right\} \preceq\left\{z_{j}^{b, 1}, z_{j}^{b, 2}\right\} \text { and }\left\{\overline{z_{j}^{b, 1}}, \overline{z_{j}^{b, 2}}\right\} \preceq X_{k}^{c} \backslash\left\{y_{j}^{d}\right\} \text {. }
$$

Furthermore, instead of $\left\{z_{i}^{e}, \overline{z_{i}^{e}}\right\}$ we add $\left\{z_{i}^{e, 1}, z_{i}^{e, 2}, \overline{z_{i}^{e, 1}}\right\},\left\{z_{i}^{e, 2}, \overline{z_{i}^{e, 1}}\right\}$ and $\left\{z_{i}^{e, 2}, \overline{z_{i}^{e, 1}}, \overline{z_{i}^{e, 2}}\right\}$. Then, the following preferences are enforced:

$$
\left\{z_{i}^{e, 1}, z_{i}^{e, 2}\right\} \prec\left\{z_{i}^{e, 1}, z_{i}^{e, 2}, \overline{z_{i}^{e, 1}}\right\} \prec\left\{z_{i}^{e, 2}, \overline{z_{i}^{e, 1}}\right\} \prec\left\{z_{i}^{e, 2}, \overline{z_{i}^{e, 1}}, \overline{z_{i}^{e, 2}}\right\} \prec\left\{\overline{z_{i}^{e, 1}}, \overline{z_{i}^{e, 2}}\right\} .
$$

Therefore, by transitivity we have

$$
X_{i}^{a} \backslash\left\{y_{j}^{b}\right\} \preceq\left\{z_{j}^{b, 1}, z_{j}^{b, 2}\right\} \prec\left\{\overline{z_{j}^{b, 1}}, \overline{z_{j}^{b, 2}}\right\} \preceq X_{k}^{c} \backslash\left\{y_{j}^{d}\right\} .
$$

and therefore $X_{i}^{a} \backslash\left\{y_{j}^{b}\right\} \prec X_{k}^{c} \backslash\left\{y_{j}^{d}\right\}$ as intended.

When constructing an order on $\mathcal{X}$ from an satisfying assignment of $\phi$ we can just replace the block $\left\{z_{i}^{e}\right\} \prec\left\{z_{i}^{e}, \overline{z_{i}^{e}}\right\} \prec\left\{\overline{z_{i}^{e}}\right\}$ resp. $\left\{\overline{z_{i}^{e}}\right\} \prec\left\{z_{i}^{e}, \overline{z_{i}^{e}}\right\} \prec\left\{z_{i}^{e}\right\}$ by the following sets $\left\{z_{i}^{e, 1}, z_{i}^{e, 2}\right\},\left\{z_{i}^{e, 1}, z_{i}^{e, 2}, \overline{z_{i}^{e, 1}}\right\},\left\{z_{i}^{e, 2}, \overline{z_{i}^{e, 1}}\right\},\left\{\overline{z_{i}^{e, 1}}, \overline{z_{i}^{e, 2}}\right\},\left\{z_{i}^{e, 2}, \overline{z_{i}^{e, 1}}, \overline{z_{i}^{e, 2}}\right\}$ ordered as demanded by dominance. It can be checked that the rest of the construction works as before, as all other new sets contain an extremum element.

\section{Strong $D I^{S}$-WO-Orderability and Strong $D I^{S} E$-WO-Orderability}

We have shown before that strong $D I^{S}$-orderability is NP-hard. However, we claim that the problem is even $\Pi_{2}^{P}$-hard, and furthermore also $\Pi_{2}^{p}$-complete. To show this we will extend the reduction used in the previous results from $\mathrm{SAT}$ to $\Pi_{2}-\mathrm{SAT}$.

Theorem 27. Strong $D I^{S}$-WO-Orderability is $\Pi_{2}^{p}$-complete.

Proof. $\Pi_{2}^{p}$-membership holds, because there is a non-deterministic Turing machine with access to an NP-oracle such that an instance $(X, \mathcal{X})$ is a yes-instance if and only if all runs of the machine end positively. This machine uses the non-determinism to guess a linear order $\leq$ on $X$ and then checks via the NP-oracle if $(X, \mathcal{X}, \leq)$ is a positive instance of $D I^{S}$-WO-Orderability, which is possible due to Theorem 25.

It remains to show that Strong $D I^{S}$-WO-Orderability is $\Pi_{2}^{p}$-hard. We do this by extending the reduction above to a reduction from a $\Pi_{2}$-SAT instance $\phi=\forall \vec{W} \exists \vec{V} \psi(\vec{W}, \vec{V})$. Recall that in the previous reductions we added for each variable $v_{i}$ two sets $X_{i}^{\mathrm{t}}$ and $X_{i}^{\mathrm{f}}$ such that the preference between these sets encoded the truth assignment of the variable $v_{i}$. Then, we ensured that a non-satisfying assignment lead to a contradiction under a specific critical linear order $\leq^{*}$. In this reduction, we also have to take care of the universally 
quantified variables $w_{1} \ldots w_{l}$. Intuitively, we want to add for every $w_{i}$ new elements $w_{i}^{\mathrm{t}}$ and $w_{i}^{\mathrm{f}}$ such that the preference between these elements determines the preference between $X_{i}^{\mathrm{t}}$ and $X_{i}^{\mathrm{f}}$, i.e., the sets encoding the truth value of $w_{i}$. Then, there is a class of critical linear orders such that every truth assignment to the universally quantified variables is encoded by at least one critical linear order. Furthermore, we will ensure that there exists an order satisfying dominance and strict independence with respect to a critical linear order $\leq$ if and only if there exists a satisfying assignment to $\psi$ that extends the assignment encoded by $\leq$.

We set up the reduction similarly to the one for Proposition 24. Additionally, we add for every universally quantified variable $w_{i}$ represented by $X_{i}^{\mathrm{t}}$ and $X_{i}^{\mathrm{f}}$ sets

$$
X_{i}^{\mathrm{t}} \backslash\left\{y_{i}^{q}\right\}, X_{i}^{\mathrm{f}} \backslash\left\{y_{i}^{q}\right\},\left\{w_{i}^{\mathrm{t}}\right\},\left\{w_{i}^{\mathrm{f}}\right\} \text { and }\left\{w_{i}^{\mathrm{t}}, w_{i}^{\mathrm{f}}\right\},
$$

where $y_{i}^{q}, w_{i}^{\mathrm{t}}$ and $w_{i}^{\mathrm{f}}$ are new elements. Then, we enforce - with the same method as before - $X_{i}^{\mathrm{t}} \backslash\left\{y_{i}^{q}\right\} \prec\left\{w_{i}^{\mathrm{t}}\right\}$ and $\left\{w_{i}^{\mathrm{f}}\right\} \prec X_{i}^{\mathrm{f}} \backslash\left\{y_{i}^{q}\right\}$ using new elements $\min _{i}^{q}$ and $\max _{i}^{q}$. This will ensure for every critical linear order $\leq^{\prime}$ with $w_{i}^{\mathrm{t}}<^{\prime} w_{i}^{\mathrm{f}}$ that $X_{i}^{\mathrm{t}} \prec X_{i}^{\mathrm{f}}$ must hold for every order $\preceq$ on $\mathcal{X}$ that satisfies dominance and strict independence with respect to $\leq$ '. We add additionally sets $X_{i}^{1} \backslash\left\{\overline{y_{i}^{q}}\right\}$ and $X_{i}^{2} \backslash\left\{\overline{y_{i}^{q}}\right\}$ and similarly enforce $X_{i}^{\mathrm{t}} \backslash\left\{\overline{y_{i}^{q}}\right\} \prec\left\{w_{i}^{\mathrm{t}}\right\}$ and $\left\{w_{i}^{\mathrm{f}}\right\} \prec X_{i}^{\mathrm{f}} \backslash\left\{\overline{y_{i}^{q}}\right\}$ using $\overline{\min _{i}^{q}}$ and $\overline{\max _{i}^{q}}$. Hence, $X_{i}^{\mathrm{t}} \prec X_{i}^{\mathrm{f}}$ must hold for every order $\preceq$ on $\mathcal{X}$ that satisfies dominance and strict independence with respect to any critical linear order $\leq^{\prime \prime}$ on $X$ with $w_{i}^{\mathrm{t}}<^{\prime \prime} w_{i}^{\mathrm{f}}$.

Now, we claim that $(X, \mathcal{X})$ can only be a positive instance of STrong $D I^{S}$-WOOrderability if $\phi$ is satisfiable. We call a linear order critical if:

- It equals the critical linear order $\leq$ in the proof of Proposition 24 on the elements already occurring in that reduction.

- The new elements $y_{i}^{q}, w_{i}^{\mathrm{t}}, w_{i}^{\mathrm{f}}, \min _{i}^{q}$ and $\max _{i}^{q}$ are added in that order as follows: $\min _{i}^{q}$ is smaller than $\min _{1}^{1}$ for all $i$. Similarly, $\max _{i}^{q}$ is larger than $\max _{m}^{3}$ for all $i$. The other elements are added between $v_{2}$ and $z_{1}^{1}$.

Then, for every truth assignment $T$ to the variables in $\vec{W}$ there is a critical linear order $\leq *$ on $X$ such that $w_{i}^{\mathrm{t}}<^{*} w_{i}^{\mathrm{f}}$ if $w_{i}$ is assigned false in $T$ and $w_{i}^{\mathrm{f}}<^{*} w_{i}^{\mathrm{t}}$ if $w_{i}$ is assigned true in $T$. Now, if there is no satisfying assignment to $\phi$ that extends $T$, then there can be no order on $\mathcal{X}$ satisfying dominance and strict independence with respect to $\leq^{*}$. Hence $(X, \mathcal{X})$ can only be $D I^{S}$-orderable with respect to every linear order $\leq^{*}$ if $\phi$ is satisfiable.

It remains to show that if $\phi$ is satisfiable then $(X, \mathcal{X})$ is a positive instance of STRONG $D I^{S}$-WO-Orderability. This can be done using nearly the same construction as above treating $X_{i}^{\mathrm{t}} \backslash\left\{y_{i}^{q}\right\}$ and $X_{i}^{\mathrm{f}} \backslash\left\{y_{i}^{q}\right\}$ as Class 2 sets, all other new sets as Class 3 sets and inserting $\left\{w_{i}^{\mathrm{t}}\right\} \prec\left\{w_{i}^{\mathrm{t}}, w_{i}^{\mathrm{f}}\right\} \prec\left\{w_{i}^{\mathrm{f}}\right\}$ resp. $\left\{w_{i}^{\mathrm{f}}\right\} \prec\left\{w_{i}^{\mathrm{t}}, w_{i}^{\mathrm{f}}\right\} \prec\left\{w_{i}^{\mathrm{t}}\right\}$ where we would insert $z_{i}^{j}$ and $\overline{z_{i}^{j}}$. The only exception has to be made if there is an $i$ such that $y_{i}^{q}=\min \left(X_{i}^{\mathrm{t}}\right)$ and $\overline{y_{i}^{q}}=\max \left(X_{i}^{\mathrm{t}}\right)$ or $\overline{y_{i}^{q}}=\min \left(X_{i}^{\mathrm{f}}\right)$ and $y_{i}^{q}=\max \left(X_{i}^{\mathrm{f}}\right)$. In the first case, we set $A \prec B$ for the sets containing $\overline{\min _{i}^{q}}$ if:

- $y_{i}^{q} \in A$ and $y_{i}^{q} \notin B$

- $y_{i}^{q} \in A, B$ or $y_{i}^{q} \notin A, B$ and $\max \left(A_{L} \triangle B_{L}\right) \in B$, 
- $y_{i}^{q} \in A, B$ or $y_{i}^{q} \notin A, B, A_{L}=B_{L}$ and $\min \left(A_{S} \triangle B_{S}\right) \in A$.

where $A_{L}:=\left\{x \in A \mid \overline{\min _{i}^{q}}<^{\prime} x\right\}$ and $A_{S}:=\left\{x \in A \mid x<^{\prime} \overline{\min _{i}^{q}}\right\}$. And for $A \prec B$ for the sets containing $\max _{i}^{q}$ if

- $\overline{y_{i}^{q}} \in B$ and $\overline{y_{i}^{q}} \notin A$

- $y_{i}^{q} \in A, B$ or $y_{i}^{q} \notin A, B$ and $\max \left(A_{L} \triangle B_{L}\right) \in B$,

- $y_{i}^{q} \in A, B$ or $y_{i}^{q} \notin A, B, A_{L}=B_{L}$ and $\min \left(A_{S} \triangle B_{S}\right) \in A$.

where $A_{L}:=\left\{x \in A \mid \max _{i}^{q}<^{\prime} x\right\}$ and $A_{S}:=\left\{x \in A \mid x<^{\prime} \max _{i}^{q}\right\}$.

It is clear that these orders satisfy dominance and strict independence, similarly to the orders on the Class 3 sets defined above. Furthermore, we have $\left\{w_{i}^{\mathrm{t}}, \max _{i}^{q}\right\} \prec\left(X_{i}^{\mathrm{t}} \backslash\left\{y_{i}^{q}\right\}\right) \cup$ $\left\{\max _{i}^{q}\right\}$ and $\left(X_{i}^{\mathrm{t}} \backslash\left\{\overline{y_{i}^{q}}\right\}\right) \cup\left\{\overline{\min _{i}^{q}}\right\} \prec\left\{w_{\mathrm{f}}, \overline{\min _{i}^{q}}\right\}$ which allows us to set $X_{i}^{\mathrm{t}} \backslash\left\{\overline{y_{i}^{q}}\right\} \prec\left\{w_{i}\right\} \prec$ $X_{i}^{\mathrm{t}} \backslash\left\{y_{i}^{q}\right\}$ which is consistent with the enforced $X_{i}^{\mathrm{t}} \backslash\left\{\overline{y_{i}^{q}}\right\} \prec X_{i}^{\mathrm{t}} \prec X_{i}^{\mathrm{f}} \backslash\left\{y_{i}^{q}\right\}$. The second case can be treated analogously.

As before in Corollary 23 and 26 we can double the elements that would otherwise appear in singletons to show the following corollary.

\section{Corollary 28. Strong $D I^{S} E$-WO-Orderability is $\Pi_{2}^{p}$-complete.}

Proof. We additionally need to double the elements $w_{i}^{\mathrm{t}}$ and $w_{i}^{\mathrm{f}}$ and add, as for $z_{i}^{a}$ and $\overline{z_{i}^{a}}$, the sets $\left\{w_{i}^{\mathrm{t}, 1}, w_{i}^{\mathrm{t}, 2}, w_{i}^{\mathrm{f}, 1}\right\},\left\{w_{i}^{\mathrm{t}, 2}, w_{i}^{\mathrm{f}, 1}\right\}$ and $\left\{w_{i}^{\mathrm{t}, 2}, w_{i}^{\mathrm{f}, 1}, w_{i}^{\mathrm{f}, 2}\right\}$. Furthermore, as before, we enforce

$$
X_{i}^{\mathrm{t}} \backslash\left\{y_{i}^{q}\right\} \preceq\left\{w_{i}^{\mathrm{t}, 1}, w_{i}^{\mathrm{t}, 2}\right\} \text { and }\left\{w_{i}^{\mathrm{t}, 1}, z_{j}^{\mathrm{t}, 2}\right\} \preceq X_{i}^{\mathrm{f}} \backslash\left\{y_{i}^{q}\right\} .
$$

Then, $w_{i}^{\mathrm{t}, 1}<w_{i}^{\mathrm{t}, 2}<w_{i}^{\mathrm{f}, 1}<w_{i}^{\mathrm{f}, 2}$, enforces $X_{i}^{\mathrm{t}} \backslash\left\{y_{i}^{q}\right\} \prec X_{i}^{\mathrm{f}} \backslash\left\{y_{i}^{q}\right\}$ and hence $X_{i}^{\mathrm{t}} \prec X_{i}^{\mathrm{f}}$. Similarly, we can ensure that $w_{i}^{\mathrm{f}, 2}<w_{i}^{\mathrm{t}, 1}<w_{i}^{\mathrm{t}, 2}<w_{i}^{\mathrm{t}, 1}$ enforces $X_{i}^{\mathrm{f}} \backslash\left\{\overline{y_{i}^{q}}\right\} \prec X_{i}^{\mathrm{t}} \backslash\left\{\overline{y_{i}^{q}}\right\}$ and hence $X_{i}^{\mathrm{f}} \prec X_{i}^{\mathrm{t}}$. Therefore, every assignment to the universally quantified variables in $\phi$ is encoded by some linear order on $X$. The rest of the proof works as before.

We conclude this part by observing that the orders constructed in the proof of Theorem 27 and Corollary 28 are not only weak but even linear orders. Therefore, the reductions used for these results show also the $\Pi_{2}^{p}$ completeness of Strong $D I^{S}$-LO-OrderabiLity and Strong $D I^{S} E$-LO-Orderability.

\section{Corollary 29. Strong $D I^{S}$-LO-Orderability and Strong $D I^{S} E$-LO-} ORDERABILITY are $\Pi_{2}^{p}$-complete.

\section{Strong $D I$-WO-Orderability and Strong DIE-WO-Orderability}

To conclude the section on $\leq$-orderability and strong orderability, we show that the hardness results we showed before also hold if we replace strict independence with regular independence. 
Theorem 30. Strong DI-WO-Orderability is $\Pi_{2}^{p}$-complete.

DI-WO-ORDERABILITY is NP-complete.

Proof. We observe that the reductions for Proposition 24 and Theorem 27 only use strict independence once: Namely when enforcing a preference $X_{i}^{a} \backslash\left\{y_{j}^{b}\right\} \preceq\left\{z_{j}^{b}\right\}$ strict independence ensures that

$$
A_{1}=\left(X_{i}^{a} \backslash\left\{y_{j}^{b}, z_{j}^{b},\right\}\right) \cup\left\{\max _{j}^{b}\right\} \prec\left\{\max _{j}^{b}\right\}=A_{l}
$$

enforces the desired preference

$$
\left(X_{i}^{a} \backslash\left\{y_{j}^{b}\right\}\right) \cup\left\{\max _{j}^{b}\right\} \prec\left\{z_{j}^{b}, \max _{j}^{b}\right\} .
$$

We can achieve the same result with dominance and independence as follows: We add the same sequence $A_{1}, \ldots, A_{l}$ as in the proof of Proposition 24 and additionally, the set $\left(X_{i}^{a} \backslash\left\{y_{j}^{b}, x_{i}^{-}\right\}\right) \cup\left\{\max _{j}^{b}\right\}$. We observe that for the sequence $A_{1}, \ldots, A_{l}$ added to enforce the preference $\left(X_{j}^{a} \backslash\left\{y_{i}^{b}\right\}\right) \cup\left\{\max _{i}^{c}\right\} \prec\left\{z_{i}, \max _{i}^{d}\right\}$, we have $l>3$ and that the following preference is enforced by dominance

$$
A_{2}=\left(X_{i}^{a} \backslash\left\{y_{j}^{b}, z_{j}^{b}, x_{i}^{-}\right\}\right) \cup\left\{\max _{j}^{b}\right\} \prec\left\{\max _{j}^{b}\right\}=A_{l}
$$

which enforces by independence

$$
\left(X_{i}^{a} \backslash\left\{y_{j}^{b}, x_{i}^{-}\right\}\right) \cup\left\{\max _{j}^{b}\right\} \prec\left\{z_{j}^{b}, \max _{j}^{b}\right\} .
$$

Now, by construction $x_{i}^{-}<\min \left(X_{i}^{a} \backslash\left\{y_{j}^{b}, x_{i}^{-}\right\}\right)$holds and hence by dominance

$$
\left.\left(X_{i}^{a} \backslash\left\{y_{j}^{b}\right\}\right) \cup\left\{\max _{j}^{b}\right\} \prec X_{i}^{a} \backslash\left\{y_{j}^{b}, x_{i}^{-}\right\}\right) \cup\left\{\max _{j}^{b}\right\} \preceq\left\{z_{j}^{b}, \max _{j}^{b}\right\} .
$$

Therefore, we get by transitivity the desired

$$
\left(X_{i}^{a} \backslash\left\{y_{j}^{b}\right\}\right) \cup\left\{\max _{j}^{b}\right\} \prec\left\{z_{j}^{b}, \max _{j}^{b}\right\} .
$$

Now, for any linear order $\leq^{\prime}$ the newly added sets can easily be added to an order satisfying dominance and independence: Any new set $\left(X_{i}^{a} \backslash\left\{y_{j}^{b}, x_{i}^{-}\right\}\right) \cup\left\{\max _{j}^{1}\right\}$ can be added in the order $\preceq$ right after $\left(X_{i}^{a} \backslash\left\{y_{j}^{b}\right\}\right) \cup\left\{\max _{j}^{1}\right\}$ if $x_{i}^{-}<^{\prime} v_{1}$ or right before $\left(X_{i}^{a} \backslash\left\{y_{j}^{b}\right\}\right) \cup\left\{\max _{j}^{1}\right\}$ if $v_{1}<^{\prime} x_{i}^{-}$.

The newly added sets are not singletons. Hence, the fact that we can add the extension rule without changing the complexity of the problem still holds as before.

Corollary 31. Strong DIE-WO-Orderability is $\Pi_{2}^{p}$-complete and DIE-WOOrderability is NP-complete.

As above, we observe that the orders constructed in Theorem 30 and Corollary 31 are linear orders. Therefore, we can also conclude that Strong DI-LO-OrderabiLity and Strong DIE-LO-Orderability are $\Pi_{2}^{p}$-complete and that $D I$-LO-Orderability and DI-LO-ORDERABILITY are NP-complete. 
Corollary 32. Strong DI-LO-Orderability and Strong DIE-LOOrderability are $\Pi_{2}^{p}$-complete. Moreover, DI-LO-OrderabiLIty and DIELO-ORDERABILITY are NP-complete.

We conclude this section with an important observation: The fact that strong orderability is $\Pi_{2}^{p}$-complete implies under the commonly believed complexity theoretic assumption coNP $\neq \Pi_{2}^{p}$ that constructing an order satisfying dominance, (strict) independence and the extension rule is hard even if we know that the family $\mathcal{X}$ is strongly orderable. In other words, even if we know that a weak order satisfying our axioms must exist, it may be hard to construct it.

Corollary 33. Assume coNP $\neq \Pi_{2}^{p}$. Then there exists no polynomial time algorithm $\mathbb{A}$ with the following specifications:

- $\mathbb{A}$ takes as input a set $X$, a family of sets $\mathcal{X} \subseteq \mathcal{P}(X)$ and a linear order $\leq$ on $X$.

- if $\mathcal{X}$ is strongly DI-orderable, then $\mathbb{A}$ produces on input $(X, \mathcal{X}, \leq)$ an order $\preceq$ on $\mathcal{X}$ that satisfies dominance and independence.

The same holds if we replace independence by strict independence or if we add the extension rule.

Proof. We claim that Strong DI-WO-Orderability would be in coNP if there exists a polynomial time algorithm $\mathbb{A}$ with the specifications above. To do so, we show that there exists a certificate for no instances if such an algorithm exists. Observe that there exists a linear order $\leq$ on $X$ that cannot be lifted if and only if $(X, \mathcal{X})$ is negative instance of Strong DI-WO-Orderability. Hence $\leq$ is a certificate (of polynomial size) for the fact that $(X, \mathcal{X})$ is a negative instance. Furthermore, one can check the certificate by running $\mathbb{A}$ on $(X, \mathcal{X}, \leq)$. Then, one only needs to check that the produced order does not satisfy dominance and strict independence. By definition, this can only be the case if $(X, \mathcal{X})$ is a negative instance of Strong $D I$-WO-Orderability. The argument for strict independence and extension is analogous.

\subsection{Partial Orders}

In this section, we investigate the effect of dropping the requirement that the lifted order should be total. For dominance and independence, we already have seen that they always can be jointly satisfied, if we expect the lifted order to be only a preorder.

Theorem 34 (Folklore). For every set $X$, linear order $\leq$ on $X$ and family of sets $\mathcal{X} \subseteq \mathcal{P}(X)$, there is a preorder that satisfies dominance, independence and the extension rule.

Proof. In Example 5, we defined the preorder $\preceq_{p m m}$ and showed that it always satisfies dominance and independence. By definition, it also satisfies the extension rule. 
In other words, every family of sets is strongly $D I(E)$-orderable if we only require the lifted order to be a preorder. On the other hand, Barbera and Pattanaik's theorem tells us that this is not the case for dominance and strict independence.

We observe that in many applications partial orders are more common than preorders. For example, if we want to avoid total orders when applying lifted orders in combinatorial voting as shown in Figure 1, then we need voting rules that can handle these incomplete orders. However, most voting rules for incomplete preferences require partial orders as input (Boutilier \& Rosenschein, 2016) though voting rules for even weaker preference models exist (Xia \& Conitzer, 2011; Terzopoulou \& Endriss, 2019). Therefore, we are often more interested in the complexity of deciding for a given family of sets if there exists a partial order that satisfies dominance and (strict) independence resp. dominance, (strict) independence and the extension rule. As strict independence and independence coincide for strict orders, we can focus just on strict independence here.

For $\leq$-orderability, this problem was already considered by Maly and Woltran (2017). They showed that it is possible to decide whether a family of sets is $\leq-D I^{S}$ - or $\leq-D I^{S} E$ orderable using a polynomial time fix point construction. In particular, this result is constructive.

Theorem 35 (Maly \& Woltran, 2017). $D I^{S}$-PO-ORDERABILITY and $D I^{S} E$-POORDERABILITY are in $\mathrm{P}$.

In this paper, we consider strongly partially $D I^{S}$-orderable families of sets, i.e., families of sets $\mathcal{X} \subseteq \mathcal{P}(X)$ such that for every linear order $\leq$ on $X$ there exists a partial order on $\mathcal{X}$ that satisfies dominance and strict independence with respect to $\leq$. It turns out that it is still difficult to decide whether a given family of sets is strongly partially $D I^{S}$-orderable. We show this by a reduction from TAUT.

Theorem 36. Strong $D I^{S}$-PO-Orderability is coNP-complete.

Proof. Let $\phi$ be an instance of TAUT, i.e., the problem of checking whether a 3-DNF is a tautology. We construct an instance $(X, \mathcal{X})$ of Strong $D I^{S}$-PO-Orderability. For every variable $X_{i}$ in $\phi$ we add new elements $x_{i}^{\mathrm{f}}$ and $x_{i}^{\mathrm{t}}$ to $X$. We call the set of these elements $V$. We will treat every order on $X$ as encoding a truth assignment by equating $x_{i}^{\mathrm{f}}<x_{i}^{\mathrm{t}}$ to $X_{i}$ is true and $x_{i}^{\mathrm{t}}<x_{i}^{\mathrm{f}}$ to $X_{i}$ is false. Then, we add for every disjunct $C_{j}$ new variables $y_{j}^{\mathrm{t}}, y_{j}^{\mathrm{f}}$. We call the set of these elements $Y$. Essentially, we want to add sets such that $\left\{y_{j}^{\mathrm{t}}\right\} \prec\left\{y_{j}^{\mathrm{f}}\right\}$ holds for the minimal partial order satisfying dominance and strict independence with respect to $\leq$ if and only if $C_{j}$ is not satisfied by the truth assignment encoded by $\leq$. Then, we will add sets that lead to a contradiction if $\left\{y_{j}^{\mathrm{t}}\right\} \prec\left\{y_{j}^{\mathrm{f}}\right\}$ holds for all disjuncts.

To achieve this, we add for every disjunct $C_{j}$ elements $c_{j}$ as well as $d_{j}^{k}$ and $e_{j}^{k}$ for $k \leq 3$. Finally, we add new elements $u, v, z_{1}$ and $z_{2}$. The elements $u$ and $v$ will be used to generate a contradiction if $\left\{y_{j}^{\mathrm{t}}\right\} \prec\left\{y_{j}^{\mathrm{f}}\right\}$ holds for all disjuncts. The elements $z_{1}$ and $z_{2}$ have the same purpose as the elements $v_{1}$ and $v_{2}$ in the proof of Proposition 21, i.e., the preference between $z_{1}$ and $z_{2}$ determines if $x_{i}^{\mathrm{f}}<x_{i}^{\mathrm{t}}$ encodes that $x_{i}$ is set to true or to false. 
Next we fix a class of linear orders on $X$ that we call critical linear orders. We want to show that for a critical linear order $\leq$ there exists a partial order on $\mathcal{X}$ satisfying dominance and strict independence only if $\leq$ encodes a satisfying truth assignment. As any possible truth assignment is encoded by a critical linear order, this means that $\mathcal{X}$ cannot be strongly partially $D I^{S}$-orderable if $\phi$ is not a tautology. We call any linear order on $X$ that is derived by replacing $V$ with an arbitrary linear order on the elements in $V$ in the following linear order a critical linear order:

$$
\begin{aligned}
& u<c_{1}<\cdots<c_{m}<y_{1}^{\mathrm{t}}<\cdots<y_{m}^{\mathrm{t}}< \\
& \qquad d_{1}^{1}<\cdots<d_{m}^{3}<V<e_{1}^{1}<\cdots<e_{m}^{3}<y_{1}^{\mathrm{f}}<\cdots< \\
& \quad y_{m}^{\mathrm{f}}<z_{1}<z_{2}<v
\end{aligned}
$$

In the following, we write $\preceq_{\infty}$ for the minimal partial order satisfying dominance and strict independence with respect to some linear order on $V$.

Next, we build the family $\mathcal{X}$. First, we make sure, that any order satisfying dominance and strict independence with respect to a critical linear order $\leq$ must reflect the truth assignment encoded by $\leq$. To this end, we add $\left\{x_{i}^{\mathrm{f}}\right\},\left\{x_{i}^{\mathrm{f}}, x_{i}^{\mathrm{t}}\right\}$ and $\left\{x_{i}^{\mathrm{t}}\right\}$ for all $x_{i}^{\mathrm{f}}, x_{i}^{\mathrm{t}} \in V$. Then, for every linear order $\leq$ we have $\left\{x_{i}^{\mathrm{f}}\right\} \prec_{\infty}\left\{x_{i}^{\mathrm{t}}\right\}$ if $x_{i}^{\mathrm{f}}<x_{i}^{\mathrm{t}}$ and, on the other hand, $\left\{x_{i}^{\mathrm{t}}\right\} \prec_{\infty}\left\{x_{i}^{\mathrm{f}}\right\}$ if $x_{i}^{\mathrm{t}}<x_{i}^{\mathrm{f}}$

Next, we add sets such that $\left\{y_{i}^{\mathrm{t}}\right\} \prec_{\infty}\left\{y_{i}^{\mathrm{f}}\right\}$ holds for a critical order $\leq$ if the assignment encoded by $\leq$ does not satisfy disjunct $C_{i}$. Essentially, we add for every variable $X_{i}$ that appears positive in disjunct $C_{j}$ a collection of sets that imply $\left\{y_{i}^{\mathrm{t}}\right\} \prec_{\infty}\left\{y_{i}^{\mathrm{f}}\right\}$ if $\left\{x_{i}^{\mathrm{t}}\right\} \prec_{\infty}\left\{x_{i}^{\mathrm{f}}\right\}$ holds. Similarly, we add a collection of sets that imply $\left\{y_{i}^{\mathrm{t}}\right\} \prec_{\infty}\left\{y_{i}^{\mathrm{f}}\right\}$ if $\left\{x_{i}^{\mathrm{f}}\right\} \prec_{\infty}\left\{x_{i}^{\mathrm{t}}\right\}$ holds for every variable that appears negatively in $C_{j}$. Now, let $C_{j}=X_{i_{1}} \wedge X_{i_{2}} \wedge X_{i_{3}}$ be a disjunct. Then, we add sets

$$
\left\{y_{j}^{\mathrm{t}}\right\},\left\{y_{j}^{\mathrm{t}}, d_{j}^{k}\right\},\left\{y_{j}^{\mathrm{t}}, d_{j}^{k}, x_{i_{k}}^{\mathrm{f}}\right\},\left\{d_{j}^{k}, x_{i_{k}}^{\mathrm{f}}\right\}
$$

for all $k \in\{1,2,3\}$ as well as

$$
\begin{aligned}
\left\{x_{i_{k}}^{\mathrm{t}}, e_{j}^{k}\right\},\left\{x_{i_{k}}^{\mathrm{t}}, e_{j}^{k}, z_{1}\right\},\left\{x_{i_{k}}^{\mathrm{t}}, e_{j}^{k}, z_{1}, z_{2}\right\}, & \left\{e_{j}^{k}, z_{1}, z_{2}\right\},\left\{e_{j}^{k}, z_{1}, z_{2}, y_{\mathrm{f}}^{j}\right\},\left\{z_{1}, z_{2}, y_{\mathrm{f}}^{j}\right\},\left\{z_{2}, y_{\mathrm{f}}^{j}\right\},\left\{y_{\mathrm{f}}^{j}\right\} .
\end{aligned}
$$

If any of the variables occurs negatively in $C_{j}$, we switch $x_{i_{k}}^{\mathrm{f}}$ and $x_{i_{k}}^{\mathrm{t}}$ in the construction. We claim that these sets ensure that $\left\{y_{j}^{\mathrm{t}}\right\} \prec_{\infty}\left\{y_{j}^{\mathrm{f}}\right\}$ holds for any critical linear order whenever at least one literal in $C_{j}$ is false. We have

$$
\left\{y_{j}^{\mathrm{t}}\right\} \prec_{\infty}\left\{y_{j}^{\mathrm{t}}, d_{j}^{k}\right\} \prec_{\infty}\left\{y_{j}^{\mathrm{t}}, d_{j}^{k}, x_{i_{k}}^{\mathrm{f}}\right\} \prec_{\infty}\left\{d_{j}^{k}, x_{i_{k}}^{\mathrm{f}}\right\} \prec_{\infty}\left\{x_{i_{k}}^{\mathrm{f}}\right\}
$$

by dominance and, hence, by transitivity $\left\{y_{j}^{\mathrm{t}}\right\} \prec_{\infty}\left\{x_{i_{k}}^{\mathrm{f}}\right\}$. Similarly, we have $\left\{x_{i_{k}}^{\mathrm{t}}\right\} \prec_{\infty}\left\{y_{j}^{\mathrm{f}}\right\}$. Hence, $\left\{x_{i_{k}}^{\mathrm{f}}\right\} \prec_{\infty}\left\{x_{i_{k}}^{\mathrm{t}}\right\}$ implies $\left\{y_{j}^{\mathrm{t}}\right\} \prec_{\infty}\left\{y_{j}^{\mathrm{f}}\right\}$ by transitivity.

Now, we add sets that lead to a contradiction if $\left\{y_{j}^{\mathrm{t}}\right\} \prec_{\infty}\left\{y_{j}^{\mathrm{f}}\right\}$ holds for all $j$ for a critical linear order $\leq$, i.e., if the assignment encoded by $\leq$ does not satisfy any disjunct. Roughly, we build a sequence of sets $A_{1}, \overline{A_{1}}, A_{2}, \overline{A_{2}}, \ldots, A_{m}, \overline{A_{m}}$ such that

1. $\{u, v\} \prec_{\infty} A_{1}$, 
2. $A_{j} \prec_{\infty} \overline{A_{j}}$ if $\left\{y_{j}^{\mathrm{t}}\right\} \prec_{\infty}\left\{y_{j}^{\mathrm{f}}\right\}$ for all $j \leq m$,

3. $\overline{A_{j}} \prec_{\infty} A_{j+1}$ for all $j<m$.

4. $\overline{A_{m}} \prec \infty\{u, v\}$,

Then, if $\left\{y_{j}^{\mathrm{t}}\right\} \prec_{\infty}\left\{y_{j}^{\mathrm{f}}\right\}$ holds for all $j$ we have $\{u, v\} \prec_{\infty}\{u, v\}$ and hence no partial order on $\mathcal{X}$ can satisfy dominance and strict independence with respect to $\leq$. For every $j \leq m$ we have:

- $A_{j}=\left\{u, c_{1}, \ldots, c_{j}, y_{j}^{\mathrm{t}}, y_{j-1}^{\mathrm{f}}, y_{j-2}^{\mathrm{f}}, \ldots, y_{1}^{\mathrm{f}}, v\right\}$

- $\overline{A_{j}}=\left\{u, c_{1}, \ldots, c_{j}, y_{j}^{\mathrm{f}}, y_{j-1}^{\mathrm{f}}, y_{j-2}^{\mathrm{f}}, \ldots, y_{1}^{\mathrm{f}}, v\right\}$

First we add

$$
\{u\},\left\{u, c_{1}\right\},\left\{u, c_{1}, y_{1}^{\mathrm{t}}\right\},\left\{u, c_{1}, y_{1}^{\mathrm{t}}, v\right\},\{u, v\} .
$$

Then, we know for any critical linear order that

$$
\{u\} \prec_{\infty}\left\{u, c_{1}\right\} \prec_{\infty}\left\{u, c_{1}, y_{1}^{\mathrm{t}}\right\}
$$

holds by dominance and therefore we have $\{u, v\} \prec_{\infty}\left\{u, c_{1}, y_{1}^{\mathrm{t}}, v\right\}$. This is the desired property (1) i.e., $\{u, v\} \prec_{\infty} A_{1}$. Now, we add for every disjunct $\left\{c_{j}, y_{j}^{\mathrm{t}}\right\}$ and $\left\{c_{j}, y_{j}^{\mathrm{f}}\right\}$. Then, we add new sets that are constructed by incrementally adding to both sets, one by one, first all elements $c_{j-1}$ to $c_{1}$, then all elements $y_{j-1}^{\mathrm{f}}$ to $y_{1}^{\mathrm{f}}$ and finally $u$ and $v$ in that order. In other words we add

$$
\begin{aligned}
& \left\{c_{j-1}, c_{j}, y_{j}^{\mathrm{t}}\right\} \text { and }\left\{c_{j-1}, c_{j}, y_{j}^{\mathrm{f}}\right\}, \\
& \left.\qquad c_{j-2}, c_{j-1}, c_{j}, y_{j}^{\mathrm{t}}\right\} \text { and }\left\{c_{j-2}, c_{j-1}, c_{j}, y_{j}^{\mathrm{f}}\right\}, \ldots, \\
& \left\{c_{1}, \ldots, c_{j}, y_{j}^{\mathrm{t}}\right\} \text { and }\left\{c_{1}, \ldots, c_{j}, y_{j}^{\mathrm{f}}\right\}
\end{aligned}
$$

as well as

$$
\begin{aligned}
&\left\{c_{1}, \ldots, c_{j}, y_{j}^{\mathrm{t}}, y_{j-1}^{\mathrm{f}}\right\} \text { and }\left\{c_{1}, \ldots, c_{j}, y_{j}^{\mathrm{f}}, y_{j-1}^{\mathrm{f}}\right\}, \ldots, \\
&\left\{c_{1}, \ldots, c_{j}, y_{j}^{\mathrm{t}}, y_{j-1}^{\mathrm{f}}, \ldots, y_{1}^{\mathrm{f}}\right\} \text { and }\left\{c_{1}, \ldots, c_{j}, y_{j}^{\mathrm{f}}, y_{j-1}^{\mathrm{f}}, \ldots, y_{1}^{\mathrm{f}}\right\}
\end{aligned}
$$

and finally

$$
\left\{u, c_{1}, \ldots, c_{j}, y_{j}^{\mathrm{t}}, y_{j-1}^{\mathrm{f}}, \ldots, y_{1}^{\mathrm{f}}\right\} \text { and }\left\{u, c_{1}, \ldots, c_{j}, y_{j}^{\mathrm{f}}, y_{j-1}^{\mathrm{f}}, \ldots, y_{1}^{\mathrm{f}}\right\}
$$

as well as

$$
\left\{u, c_{1}, \ldots, c_{j}, y_{j}^{\mathrm{t}}, y_{j-1}^{\mathrm{f}}, \ldots, y_{1}^{\mathrm{f}}, v\right\} \text { and }\left\{u, c_{1}, \ldots, c_{j}, y_{j}^{\mathrm{f}}, y_{j-1}^{\mathrm{f}}, \ldots, y_{1}^{\mathrm{f}}, v\right\}
$$

By construction

$$
\left\{u, c_{1}, \ldots, c_{j}, y_{j}^{\mathrm{t}}, y_{j-1}^{\mathrm{f}}, \ldots, y_{1}^{\mathrm{f}}, v\right\} \prec_{\infty}\left\{u, c_{1}, \ldots, c_{j}, y_{j}^{\mathrm{f}}, y_{j-1}^{\mathrm{f}}, \ldots, y_{1}^{\mathrm{f}}, v\right\}
$$


holds for the minimal partial order satisfying dominance and strict independence for any linear order on $V$ if and only if $\left\{y_{j}^{\mathrm{t}}\right\} \prec_{\infty}\left\{y_{j}^{\mathrm{f}}\right\}$ holds for that partial order. This is the desired property (2) of the sequence.

Next, we add the following sets:

$$
\left\{u, c_{1}, \ldots, c_{j}\right\},\left\{u, c_{1}, \ldots, c_{j+1}\right\} \text { and }\left\{u, c_{1}, \ldots, c_{j+1}, y_{j+1}^{\mathrm{t}}\right\} \text {. }
$$

Then, we add new sets derived as above by adding to both sets first all elements $y_{j}^{\mathrm{f}}$ to $y_{1}^{\mathrm{f}}$ and then $v$, one by one, in that order until we reach

$$
\left\{u, c_{1}, \ldots, c_{j}, y_{j}^{\mathrm{f}}, y_{j-1}^{\mathrm{f}}, \ldots, y_{1}^{\mathrm{f}}, v\right\} \text { and }\left\{u, c_{1}, \ldots, c_{j+1}, y_{j+1}^{\mathrm{t}}, y_{j}^{\mathrm{f}}, y_{j-1}^{\mathrm{f}}, \ldots, y_{1}^{\mathrm{f}}, v\right\} .
$$

Then, the desired property (3)

$$
\left\{u, c_{1}, \ldots, c_{j}, y_{j}^{\mathrm{f}}, y_{j-1}^{\mathrm{f}}, \ldots, y_{1}^{\mathrm{f}}, v\right\} \prec_{\infty}\left\{u, c_{1}, \ldots, c_{j+1}, y_{j+1}^{\mathrm{t}}, y_{j}^{\mathrm{f}}, y_{j-1}^{\mathrm{f}}, \ldots, y_{1}^{\mathrm{f}}, v\right\}
$$

holds for the critical linear order by strict independence because

$$
\left\{u, c_{1}, \ldots, c_{j}\right\} \prec\left\{u, c_{1}, \ldots, c_{j+1}\right\} \prec\left\{u, c_{1}, \ldots, c_{j+1}, y_{j+1}^{\mathrm{t}}\right\}
$$

holds by dominance. Finally, we add $\{v\}$ and then $\left\{y_{1}^{\mathrm{f}}, v\right\},\left\{y_{2}^{\mathrm{f}}, y_{1}^{\mathrm{f}}, v\right\}$ and so on till we reach

$$
\left\{c_{1}, \ldots, c_{m}, y_{m}^{\mathrm{f}}, y_{m-1}^{\mathrm{f}}, \ldots, y_{1}^{\mathrm{f}}, v\right\} .
$$

This forces for any critical linear order the desired property (4):

$$
\left\{u, c_{1}, \ldots, c_{m}, y_{m}^{\mathrm{f}}, y_{m-1}^{\mathrm{f}}, \ldots, y_{1}^{\mathrm{f}}, v\right\} \prec_{\infty}\{u, v\} .
$$

Now, by construction and transitive we have for any critical linear order

$$
\begin{aligned}
&\{u, v\} \prec_{\infty}\left\{u, c_{1}, y_{1}^{\mathrm{t}}, v\right\} \prec_{\infty} \\
&\left\{u, c_{1}, y_{1}^{\mathrm{f}}, v\right\} \prec_{\infty}\left\{u, c_{1}, c_{2}, y_{2}^{\mathrm{t}}, y_{1}^{\mathrm{f}}, v\right\} \prec_{\infty} \ldots \\
& \prec_{\infty}\left\{u, c_{1}, \ldots, c_{m}, y_{m}^{\mathrm{f}}, y_{m-1}^{\mathrm{f}}, \ldots, y_{1}^{\mathrm{f}}, v\right\} \prec_{\infty}\{u, v\}
\end{aligned}
$$

if (and only if) $\left\{y_{j}^{\mathrm{t}}\right\} \prec_{\infty}\left\{y_{j}^{\mathrm{f}}\right\}$ holds for all disjuncts, i.e., if the critical linear order encodes an unsatisfying assignment. It follows that if $\phi$ is not a tautology, then $(X, \mathcal{X})$ is not strongly partial $D I^{S}$-orderable.

It remains to show that $(X, \mathcal{X})$ is strongly partial $D I^{S}$-orderable if $\phi$ is a tautology. Let $\leq$ be a linear order on $X$. As in the proof of Proposition 21 we can assume, by Lemma 8, that $z_{1}<z_{2}$. We construct a partial order $\preceq$ that satisfies dominance and strict independence with respect to $\leq$. To avoid complicated case distinctions, we will describe the construction only for disjuncts with all positive variables. The only change in construction required for negative variables is switching $x_{i}^{\mathrm{f}}$ and $x_{i}^{\mathrm{t}}$.

First we add the forced preferences between $\left\{x_{i}^{\mathrm{f}}\right\},\left\{x_{i}^{\mathrm{f}}, x_{i}^{\mathrm{t}}\right\}$ and $\left\{x_{i}^{\mathrm{t}}\right\}$, i.e., $\left\{x_{i}^{\mathrm{f}}\right\} \prec\left\{x_{i}^{\mathrm{f}}, x_{i}^{\mathrm{t}}\right\} \prec$ $\left\{x_{i}^{\mathrm{t}}\right\}$ if $x_{i}^{\mathrm{f}}<x_{i}^{\mathrm{t}}$ holds and $\left\{x_{i}^{\mathrm{t}}\right\} \prec\left\{x_{i}^{\mathrm{f}}, x_{i}^{\mathrm{t}}\right\} \prec\left\{x_{i}^{\mathrm{f}}\right\}$ if $x_{i}^{\mathrm{t}}<x_{i}^{\mathrm{f}}$ holds. Next, we consider the sets containing an element $d_{j}^{k}$. We add all preferences that are implied by dominance between the following sets:

$$
\left\{y_{j}^{\mathrm{t}}\right\},\left\{y_{j}^{\mathrm{t}}, d_{j}^{k}\right\},\left\{y_{j}^{\mathrm{t}}, d_{j}^{k}, x_{i_{k}}^{\mathrm{f}}\right\},\left\{d_{j}^{k}, x_{i_{k}}^{\mathrm{f}}\right\},\left\{x_{i_{k}}^{\mathrm{f}}\right\}
$$


Then we close under transitivity. We recall that applying dominance and transitivity can never lead to a contradiction. Furthermore, we claim that $\prec$ restricted to these sets already satisfies strict independence: The only possible application of strict independence on these sets is that any preference between $\left\{y_{j}^{\mathrm{t}}\right\}$ and $\left\{x_{i_{k}}^{\mathrm{f}}\right\}$ has to be lifted to $\left\{y_{j}^{\mathrm{t}}, d_{j}^{k}\right\}$ and $\left\{d_{j}^{k}, x_{i_{k}}^{\mathrm{f}}\right\}$. By construction however, there can only be a preference between $\left\{y_{j}^{\mathrm{t}}\right\}$ and $\left\{x_{i_{k}}^{\mathrm{f}}\right\}$ forced by dominance and transitivity if the same preference holds between $\left\{y_{j}^{\mathrm{t}}, d_{j}^{k}\right\}$ and $\left\{d_{j}^{k}, x_{i_{k}}^{\mathrm{f}}\right\}$ as for $\left\{y_{j}^{\mathrm{t}}\right\}$ dominance can only force a relation to $\left\{y_{j}^{\mathrm{t}}, d_{j}^{k}\right\}$ and for $\left\{x_{i_{k}}^{\mathrm{f}}\right\}$ it can only force a relation to $\left\{d_{j}^{k}, x_{i_{k}}^{\mathrm{f}}\right\}$. Moreover, because we assume that no variable occurs twice in a disjunct, a preference between $\left\{y_{j}^{\mathrm{t}}\right\}$ and $\left\{x_{i_{k}}^{\mathrm{f}}\right\}$ cannot later be introduced through sets containing another $d_{j^{\prime}}^{k^{\prime}}$. Indeed the only preferences we need to add for sets containing different elements $d_{j_{1}}^{k_{1}}$ and $d_{j_{2}}^{k_{2}}$, in order to satisfy dominance and transitivity is $\left\{x_{i}^{\mathrm{f}}, d_{j_{1}}^{k_{1}}\right\} \prec\left\{x_{i}^{\mathrm{f}}, d_{j_{2}}^{k_{2}}\right\}$ for all $x_{i}^{\mathrm{f}}$ and all $d_{j_{1}}^{k_{1}}, d_{j_{2}}^{k_{2}}$ such that $d_{j_{1}}^{k_{1}}<d_{j_{2}}^{k_{2}}$ holds.

Using a similar construction, we can order all sets containing an element $e_{j}^{k}$ if we replace $x_{i}^{\mathrm{f}}$ by $x_{i}^{\mathrm{t}}$ and $y_{j}^{\mathrm{t}}$ by $\left\{z_{1}, z_{2}, y_{j}^{\mathrm{f}}\right\}$. Finally, we add the enforced preference between $\left\{z_{2}, y_{j}^{\mathrm{f}}\right\}$ and $\left\{y_{j}^{\mathrm{f}}\right\}$ as well as $\left\{z_{1}, z_{2}, y_{j}^{\mathrm{f}}\right\} \prec\left\{z_{2}, y_{j}^{\mathrm{f}}\right\}$. The later is enforced by dominance as we assume $z_{1}<z_{2}$. Then, we close everything under transitivity. By construction, this does not produce any new instances of strict independence.

We now consider the sets containing an element $c_{i}$ for some $i$. Observe that $\left\{z_{1}, z_{2}, y_{j}^{\mathrm{f}}\right\} \prec$ $\left\{z_{2}, y_{j}^{\mathrm{f}}\right\}$ implies that only $\left\{x_{i_{k}}^{\mathrm{t}}\right\} \prec\left\{y_{j}^{\mathrm{f}}\right\}$ can be forced but not $\left\{y_{j}^{\mathrm{f}}\right\} \prec\left\{x_{i_{k}}^{\mathrm{t}}\right\}$. This implies that $\left\{y_{j}^{\mathrm{f}}\right\} \prec\left\{y_{j}^{\mathrm{t}}\right\}$ never holds. Moreover, $\left\{y_{j}^{\mathrm{t}}\right\} \prec\left\{y_{j}^{\mathrm{f}}\right\}$ can only be enforced if $\left\{x_{i}^{\mathrm{f}}\right\} \prec\left\{x_{i}^{\mathrm{t}}\right\}$ holds for a variable occurring in disjunct $C_{j}$ i.e., if disjunct $C_{j}$ is not satisfied. As $\phi$ is a tautology, there is disjunct $C_{l}$ that is satisfied by the assignment encoded by $\leq$. Hence, $\left\{y_{l}^{\mathrm{t}}\right\}$ and $\left\{y_{l}^{\mathrm{f}}\right\}$ are incomparable. We partition the sets containing an element $c_{i}$ in partitions $P_{1}, \ldots, P_{m}$ based on the largest $i$ for which they contain $c_{i}$. We set $S_{1} \prec S_{2}$ if $S_{1} \in P_{i_{1}}$, $S_{2} \in P_{i_{2}}$ and one of the following holds:

- $c_{i_{1}}<c_{i_{2}}$ and $i_{1}, i_{2}<l$

- $c_{i_{1}}<c_{i_{2}}$ and $l<i_{1}, i_{2}$

Any set that contains $c_{i}$ also contains $y_{i}$ except $\left\{u, c_{1}, \ldots, c_{i}\right\}$. Hence, all sets from different partitions differ by at least two elements except $\left\{u, c_{1}, \ldots, c_{i}\right\}$ and $\left\{u, c_{1}, \ldots, c_{i+1}\right\}$. If dominance forces a preference between these sets, it is satisfied by construction for $i, i+1 \neq l$. Now, for any set in any partition $P_{i}$ such that $i \neq l$ we set $S \prec S^{\prime}$ if $y_{i}^{\mathrm{t}} \in S$ and $y_{i}^{\mathrm{t}} \notin S^{\prime}$. This covers all applications of strict independence in a partition. Finally, we add all preferences that are forced by dominance in a partition and close under transitivity. We observe that $S, S \cup\{x\} \in P_{i}$ implies either $y_{i}^{\mathrm{t}} \in S, S \cup\{x\}$ or $y_{i}^{\mathrm{t}} \notin S, S \cup\{x\}$, hence this cannot lead to a contradiction. Now, for a set $S$ in $P_{l}$ such that $y_{l}^{\mathrm{t}} \in S$ we set

- $S^{\prime} \prec S$ if $S^{\prime} \in P_{i}$ for $i<l$ and $c_{i}<c_{l}$

- $S \prec S^{\prime}$ if $S^{\prime} \in P_{i}$ for $i<l$ and $c_{l}<c_{i}$

Furthermore, for a set $S$ in $P_{l}$ such that $y_{l}^{\mathrm{t}} \notin S$ we set

- $S^{\prime} \prec S$ if $S^{\prime} \in P_{i}$ for $l<i$ and $c_{i}<c_{l}$ 
- $S \prec S^{\prime}$ if $S^{\prime} \in P_{i}$ for $l<i$ and $c_{l}<c_{i}$

And finally, we add again all preferences forced by dominance and close by transitivity. As $\left\{y_{l}^{\mathrm{t}}\right\}$ and $\left\{y_{l}^{\mathrm{f}}\right\}$ are incomparable in $\preceq$ this order is consistent. Furthermore, we did not add any preferences between any set not containing $c_{l}$ and sets containing $c_{l+1}$. Hence, $\left\{u, c_{1}, y_{1}^{\mathrm{t}}, z_{1}, z_{2}, v\right\}$ and $\left\{u, c_{1}, \ldots, c_{m}, y_{m}^{\mathrm{f}}, \ldots, y_{1}^{\mathrm{f}}, v\right\}$ are incomparable in $\preceq$. This allows us to add any preferences forced by dominance and strict independence regarding $\{u\},\{v\}$ and $\{u, v\}$ without creating a contradiction. By construction, $\preceq$ is now a partial order that satisfies dominance and strict independence.

We can extend the result to Strong $D I^{S} E$-PO-OrderabiLity using the same idea of doubling the singletons as before.

Corollary 37. Strong $D I^{S}$ E-PO-Orderability is coNP-complete.

Proof. Strong $D I^{S} E$-PO-Orderability is in conP by the same argument as Strong $D I^{S}$-PO-ORDERABILITy. We modify the reduction used to prove Theorem 36 to show that it is also coNP-hard. All singletons appearing in that reduction are of the form $\left\{x_{i}^{\mathrm{t}}\right\},\left\{x_{i}^{\mathrm{f}}\right\}$, $\left\{y_{i}^{\mathrm{t}}\right\},\left\{y_{i}^{\mathrm{f}}\right\}\{u\}$ or $\{v\}$ for some $i$. We change the reduction in a way that only singletons of the form $\{u\}$ or $\{v\}$ appear. As before, we can achieve this by doubling the elements of the form $x_{i}^{a}$ and $y_{i}^{a}$ for $a \in\{\mathrm{t}, \mathrm{f}\}$, that means we replace $x_{i}^{a}$ by two elements $x_{i}^{a, 1}$ and $x_{i}^{a, 2}$, and replace $y_{i}^{a}$ by $y_{i}^{a, 1}$ and $y_{i}^{a, 2}$. As in the proof of Corollary 28, we add the sets $\left\{x_{i}^{\mathrm{t}, 1}, x_{i}^{\mathrm{t}, 2}, x_{i}^{\mathrm{f}, 1}\right\},\left\{x_{i}^{\mathrm{t}, 2}, x_{i}^{\mathrm{f}, 1}\right\}$ and $\left\{x_{i}^{\mathrm{t}, 2}, x_{i}^{\mathrm{f}, 1}, x_{i}^{\mathrm{f}, 2}\right\}$. Then $x_{i}^{\mathrm{t}, 1}<x_{i}^{\mathrm{t}, 2}<x_{i}^{\mathrm{f}, 1}<x_{i}^{\mathrm{f}, 2}$ implies $\left\{x_{i}^{\mathrm{t}, 1}, x_{i}^{\mathrm{t}, 2}\right\} \prec\left\{x_{i}^{\mathrm{f}, 1}, x_{i}^{\mathrm{f}, 2}\right\}$. and $x_{i}^{\mathrm{t}, 1}>x_{i}^{\mathrm{t}, 2}>x_{i}^{\mathrm{f}, 1}>x_{i}^{\mathrm{f}, 2}$ implies $\left\{x_{i}^{\mathrm{t}, 1}, x_{i}^{\mathrm{t}, 2}\right\} \succ\left\{x_{i}^{\mathrm{f}, 1}, x_{i}^{\mathrm{f}, 2}\right\}$. Furthermore, we add $\left\{y_{j}^{\mathrm{t}, 2}, d_{j}^{k}\right\},\left\{d_{j}^{k}, x_{i}^{\mathrm{t}, 1}\right\},\left\{x_{i}^{\mathrm{f}, 2}, e_{j}^{k}\right\}$ and $\left\{z_{2}, y_{j}^{\mathrm{t}, 1}\right\}$ if variable $X_{i}$ appears positively in disjunct $j$. If $X_{i}$ appears negatively, we switch $\mathrm{t}$ and $\mathrm{f}$. Then, $\left\{x_{i}^{\mathrm{t}, 1}, x_{i}^{\mathrm{t}, 2}\right\} \prec$ $\left\{x_{i}^{\mathrm{f}, 1}, x_{i}^{\mathrm{f}, 2}\right\}$ implies $\left\{y_{j}^{\mathrm{t}, 1}, y_{j}^{\mathrm{t}, 2}\right\} \prec\left\{y_{j}^{\mathrm{f}, 1}, x_{i}^{\mathrm{f}, 2}\right\}$ as desired.

The second part of the construction must be modified by always adding first $y_{j}^{\mathrm{t}, 1}$ and then $y_{j}^{\mathrm{t}, 2}$ instead of $y_{j}^{\mathrm{t}}$ and similarly $y_{j}^{\mathrm{f}, 1}$ and then $y_{j}^{\mathrm{f}, 2}$ instead of $y_{j}^{\mathrm{f}}$. It can be checked that this suffices to force $\{u, v\} \prec\{u, v\}$ whenever no disjunct is satisfied.

Now, constructing a partial order that satisfies dominance and strict independence works as before. Furthermore, $\{u\}$ and $\{v\}$ are the only singletons in the family $\mathcal{X}$. As $\{u, v\} \in \mathcal{X}$, any partial order that satisfies dominance also satisfies the extension rule.

As mentioned before, independence and strict independence coincide in the case of partial orders. This may be problematic if one wishes to use lifted orders in an application where pre-orders can not be used, for example, if one wants to combine lifted orders with a voting rule that takes partial orders as input. If in such a situation strict independence either turns out to be not satisfiable in conjunction with dominance or it is considered to restrictive, it may seem at first that the order lifting approach can not be used at all. However, it is possible to define a weaker version of independence that provides a different interpretation of the monotonicity idea in the case of partial orders.

Axiom 38 (Weak independence). For all $A, B \in \mathcal{X}$ and for all $x \in X \backslash(A \cup B)$ such that $A \cup\{x\}, B \cup\{x\} \in \mathcal{X}:$

$$
A \prec B \text { implies } B \cup\{x\} \nprec A \cup\{x\} \text {. }
$$


Clearly, any total relation satisfies weak independence if and only if it satisfies independence. However, in contrast to strict independence and independence it is always possible to find a partial order that satisfies dominance and weak independence.

Proposition 39. Let $X$ be a set, $\leq$ a linear order on $X$ and $\mathcal{X} \subseteq \mathcal{P}(X) \backslash\{\emptyset\}$. Furthermore, let $\preceq$ be a preorder on $\mathcal{X}$ that satisfies dominance and independence. Then, the corresponding strict order is an antisymmetric, irreflexive and transitive binary relation that satisfies dominance and weak independence.

Proof. By definition the corresponding strict order $\prec_{s}$ of $\preceq$ is an antisymmetric, irreflexive, transitive relation. Furthermore, by the definition of dominance, $\preceq$ satisfies dominance if and only if $\prec_{s}$ satisfies dominance. Now assume $A, B, A \cup\{x\}, B \cup\{x\} \in \mathcal{X}$ and $A \prec_{s} B$. Then also $A \prec B$ must hold. Hence, by independence either $A \cup\{x\} \sim B \cup\{x\}$ or $A \cup\{x\} \prec B \cup\{x\}$. In both cases we have $B \cup\{x\} \nprec_{s} A \cup\{x\}$. Therefore, $\prec_{s}$ satisfies weak independence.

If we add to $\prec_{s}$ the preference $A \sim A$ for all $A \in \mathcal{X}$ we get a partial order that satisfies dominance and weak independence.

\subsection{Succinct Domain Restrictions}

The results in the previous sections assume that the family of sets is given explicitly. However, in many applications, the family of sets is instead only given implicitly, via some condition that has to be satisfied by the sets in order to be admissible. In this section, we consider a specific, well studied succinct representation - families represented by boolean circuits - and show that it can lead to a massive blow up in complexity. While boolean circuits are not commonly used for succinct representation in practice, they are a useful tool to determine the effect that succinct representation can have in the worst case as they are very expressive. In particular, boolean circuits can simulate many succinct representation that are commonly used in practice, like propositional formulas or knowledge compilation languages such as NNFs (Darwiche \& Marquis, 2002). This worst case behavior is especially interesting in the light of the results of Maly et al. (2019) which show that many of the studied problems become polynomial time solvable if the families of sets is represented as the set of connected subgraphs of a graph. Now, how can boolean circuits be used for succinct representation? Consider for example the following, simple family:

$$
\{1,2\},\{1,2,4\}
$$

Assume that we know that we have a family of sets over four elements. Then the family above can represented by the boolean string 11001101 . This string in turn can be represented by a boolean circuit, such that on the input of the number $i-1$ (in binary) the circuit outputs the $i$-th bit of the string. The circuit pictured in Figure 4 is an example for a circuit representing the string 11001101.

In general, this representation can be exponentially smaller than the string (and hence the family of sets), as we only need $\log (n)$ many input gates to represent a string of length $n$. However, while every string of length $n$ can be represented by a boolean circuit with $n$ gates only strings with a enough "logical structure" can be succinctly represented using only $O(\log (n))$ logical gates in addition to the $\log (n)$ input gates. 


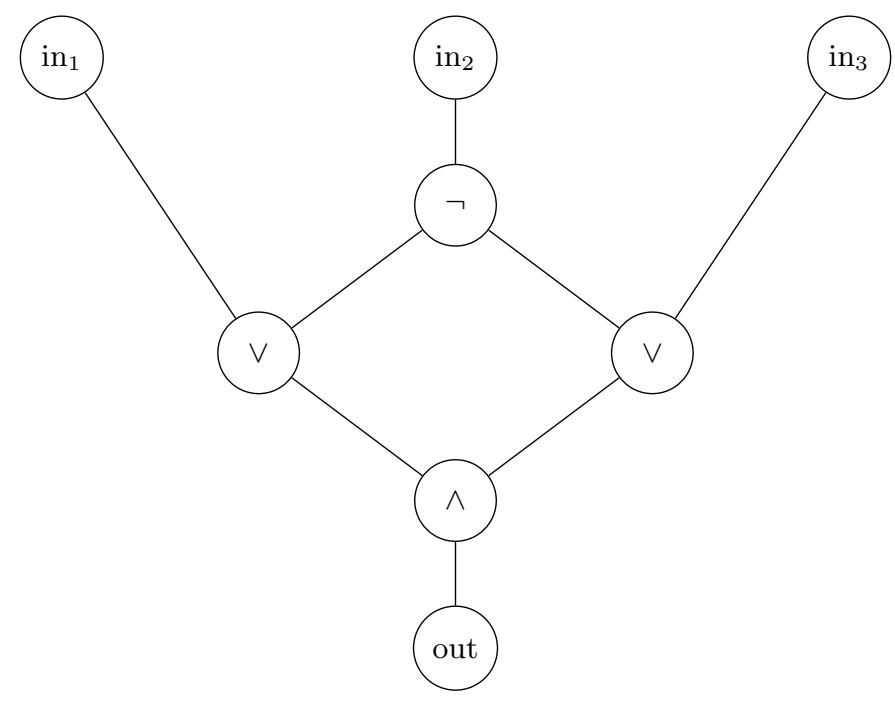

Figure 4: A boolean circuit representing the string 11001101

Before we show our results, we quickly review the basic results on succinctly represented problems from the literature and recall the definitions and lemmas we need. The study of succinct problems goes back to Galperin and Wigderson (1983) for graphs that are succinctly represented by a boolean circuit. Later this approach was extended by Balcázar, Lozano, and Torán (1992) to arbitrary problems that are succinctly represented by boolean circuits in the following way.

Definition 40. We say a boolean circuit $C_{w}$ with two output gates represents a binary string $w$ if for every input of a binary number $i$ the following holds:

- the first output is 1 if and only if $i \leq|w|$

- if the first output is 1 then the second output equals the $i$-th bit of $w$.

The succinct version $Q_{S}$ of a problem $Q$ is: Given a boolean circuit $C_{w}$ representing a boolean string $w$ decide whether $w \in Q$.

For example, Succinct SAT - the succinct version of SAT - can be defined as follows:

\section{SuCCINCT SAT}

Input: $\quad$ A boolean circuit $C_{w}$ representing a word $w$.

Question: Is the 3 -CNF represented by $w$ satisfiable? ${ }^{9}$

Succinct SAT has been shown to be NEXP-complete (Papadimitriou \& Yannakakis, 1986; Papadimitriou, 1994). Hence, Succinct TAut is coNEXP-complete. Succinct versions of the problems considered in this paper be can defined similarly. The main tool to determine the complexity of succinct problems are so-called Conversion Lemmas. We use

9. It is not important what specific encoding is used as long as the number of variables and clauses as well as the $\mathrm{i}$-th variable in the $\mathrm{j}$-th clause can be read in polylog-time. Any reasonable encoding will satisfy this requirement. 
the Conversion Lemma by Balcázar et al. (1992). Stronger versions of this lemma exist, for example by Veith (1998). However, the Conversion Lemma of Balcázar et al. (1992) suffices for our purposes and has the advantage that only comparably simple reductions are used, namely ptime reductions and polylog-time reductions. polylog-time reductions are reductions that - given random access to the input - need only $O\left(\log ^{c}(n)\right)$-time to output an arbitrary bit of the output. The following definition is taken from (Murray \& Williams, 2017).

Definition 41. An algorithm $R:\{0,1\}^{*} \times\{0,1\}^{*} \rightarrow\{0,1, \star\}$ is a polylog-time reduction from $L$ to $L^{\prime}$ if there are constants $c \geq 1$ and $k \geq 1$ such that for all $x \in\{0,1\}^{*}$,

- $R(x, i)$ has random access to $x$

- $R(x, i)$ runs in $O\left((\log (|x|))^{k}\right)$ time for all $i \in\{0,1\}^{\lceil 2 c \log (|x|)\rceil}$

- there is an $l_{x} \leq|x|^{c}+c$ such that $R(x, i) \in\{0,1\}$ and for all $i \leq l_{x}$ and $R(x, i)=\star$ for all $i>l_{x}$.

- $x \in L$ iff $R(x, 1) \cdot R(x, 2) \cdots R\left(x, l_{x}\right) \in L^{\prime}$.

Here $\cdot$ is the string concatenation and $\star$ is the out of bounds character that marks the end of a string. Now, we can formulate the Conversion Lemma of Balcázar et al. (1992).

Lemma 42 (Conversion Lemma). Let $Q$ and $Q^{*}$ be decision problems. If there is a polylog-time reduction from $Q$ to $Q^{*}$ then there is a ptime reduction from $Q_{S}$ to $Q_{S}^{*}$.

We can use the Conversion Lemma to prove the following theorem.

Theorem 43. SuCCINCT $D I^{S}$-LO-ORderability is NEXP-complete. SuCCINCT STRONG $D I^{S}$-LO-ORDERABILITY is NEXP-hard. The same holds if we add the extension rule.

Proof. Succinct $D I^{S}$-LO-Orderability can be solved in NEXP-time by explicitly computing the family $\mathcal{X}$ and then solving the (exponentially larger) explicit problem in NP-time.

For the hardness, we only have to check that the presented reduction is computable in polylog-time. Then, by the Conversion Lemma, there is a ptime reduction from SucCINCT SAT to the problems mentioned above. The NEXP-hardness of both problems then follows as Succinct SAT is known to be NEXP-complete. We have to show that we can compute a single bit of the output in polylog-time if we have random access to the input. For this, we have to take the binary representation of SAT into account. Unfortunately, neither Papadimitriou and Yannakakis (1986) nor Papadimitriou (1994) specify a binary representation for the NEXP-hardness proof. However, the proof of NEXP-hardness is not sensitive to the representation as long as it is reasonable. The same is true for this proof. Reasonable means in our context that it is possible to determine the number of variables $n$ and clauses $m$ in polylog-time. For any sensible encoding of 3-CNF this is either explicitly encoded or can be determined via binary search. Furthermore, we assume that one only needs polylog-time to read the $\mathrm{i}$-th variable in the $\mathrm{j}$-th clause. This is trivially true if we assume that every clause is encoded by the same amount of bits. An example of an encoding that is reasonable in our sense is the following encoding: The encoding starts with the number of variables $n$ encoded in binary. Then, the formula is encoded as follows 
- Each variable is encoded by a unique string of length $\log (n)+1$.

- Furthermore, let $s_{0}$ be a string of length $\log (n)+1$ that does not encode a variable.

- Each block of $3(\log (n)+1)+3$ bits encodes one clause $C_{j}$, the first bit determines whether the first variable appears positively or negatively in $C_{j}$ and the next $\log (n)+1$ equal the encoding of the first variable. The remaining literals are encoded similarly. If the clause contains less than 3 literals, then the empty slots are filled with $s_{0}$.

It is easy to see that the proof by Papadimitriou (1994) of the NEXP-hardness of SUCCINCT SAT works for such an encoding.

Now, we fix a binary representation for instances of $D I^{S}$-LO-ORDERABILITY resp. Strong $D I^{S}$-LO-orderability. First, we encode the number of elements $k$ of $X$ in binary. Then, the family $\mathcal{X}$ is encoded as a series of strings of length $k$, where a 1 in position $l$ means the $l$-th element of $X$ is in the set and a 0 in position $l$ means the $l$-th element is not in the set. For an instance of $D I^{S}$-LO-ORDERABILITY, the linear order $\leq$ is given by the natural order on these positions.

First, observe that the size of $X$ is $4 n+12 m+3$ and the size of $\mathcal{X}$ is $p(n, m)$ for some polynomial $p(x, y)$. Therefore, we can determine it in polylog-time. Now, assume we want to decide whether the $i$-th bit of the output is 0 or 1 . It is clear that this can be done in polylog-time if the $i$-th bit is part of the representation of the size of $X$. Assume that the $i$-th bit determines if the $l$-th element $x$ is part of a $k$-th set $A$. We can assume that we fixed an order in which we generate the sets in $\mathcal{X}$ such that we can compute from $m, n$ and $i$ which set $A$ is supposed to be. Say we first compute the Class 1 sets. For these, we only need to know the number of variables in the SAT instance. Next, we compute the Class 2 sets. Observe that, for a fixed number of variables and clauses, the only elements that depends on the SAT instance are the elements of the form $x_{j}^{+}$or $x_{j}^{-}$. For these elements, it suffices to know if $v_{j}$ occurs (positively or negatively) in a specific clause in the right position. In our exemplary encoding of SAT it suffices to read $\log (n)+2$ bits to determine this. The position of these bits only depends on which position in which clause we want to check. Finally, the Class 3 sets are all either of the form $\left\{z_{i}^{a}\right\},\left\{z_{i}^{a}, \min _{i}^{a}\right\} \operatorname{resp.}\left\{z_{i}^{a}, \min _{i}^{a}\right\}$ or they equal a Class 2 set with a specific number of elements removed. In the first case, the elements of of the set again only depend on the number of variables and clauses and in the second case the elements can be computed the same way as a Class 2, as the removed elements also only depend on on the number of variables and clauses.

We observe that the argument above does not use any properties of the reduction that are unique to $D I^{S}$-LO-orderability. Therefore, it is straightforward to check that the hardness of the other strong and $\leq$-orderability properties can be lifted in the same way. Moreover, NEXP-membership follows for all $\leq$-orderability problems by the same argument as above.

Corollary 44. Succinct DI-LO-Orderability, Succinct $D I$-WOOrderability, Succinct $D I^{S}$-WO-Orderability are NEXP-complete. Succinct Strong $D I$-LO-Orderability, Succinct Strong $D I$-WO-Orderability SucCINCT STRONG $D I^{S}$-WO-ORDERABILITy are NEXP-hard. The same holds if we additionally add the extension rule. 
Moreover, we note that the Conversion Lemma can also be applied the same way to the reduction from TAut to Strong PARTial $D I^{S}$-ORDERABILITY.

Theorem 45. Succinct Strong $D I^{S}$-PO-Orderability is coNEXP-complete.

This analysis still leaves some gaps. It can be shown that Succinct Strong $D I^{S_{-}}$ WO-ORDERABILITY is in $\Pi_{2}^{E}$, the second level of the exponential hierarchy, by a similar argument as the one used to show that SUCCINCT $D I^{S}$-WO-ORDERABILITY is in NEXP. It seems very likely that this upper bound is tight and that SuCCINCT STRONG $D I^{S}$-WOOrderABILITY is indeed $\Pi_{2}^{E}$-complete. However, as the succinct version of $\Pi^{2}$-SAT is, to the best of our knowledge, not known to be $\Pi_{2}^{E}$-hard, the Conversion Lemma does not suffice to show this. Closing this gap is, therefore, left to future work. The same holds for the other problems regarding strong orderability where the lifted order needs to be a linear or weak order.

On the other hand, we do not provide a lower bound for Succinct $D I^{S}(E)$-POORDERABILITY, because we do not have a lower bound on the complexity of $D I^{S}(E)$ PO-ORDERABILITY even in the non-succinct case. We would conjecture that $D I^{S}(E)$-POOrderabiLity is P-complete, but leave a proof of this to future work.

\subsection{Weak Orderability}

In this section, we consider weak orderability. We will restrict our attention to weak orderability with respect to dominance and strict independence, resp. with respect to dominance, strict independence and the extension rule. The question if these results also hold for regular independence is left for future work. First, we show that WeaK $D I^{S}$-WO-OrderabiLITy is NP-complete. This requires a completely different construction. We reduce this time from Betweenness which was shown to be NP-hard by Opatrny (1979).

\section{BETWEENNESS}

Input: $\quad$ A set $V=\left\{v_{1}, v_{2}, \ldots, v_{n}\right\}$ and a set of triples $R \subseteq V^{3}$.

Question: Does there exist a linear order on $V$ such that $a<b<c$ or $a>b>c$ holds for all $(a, b, c) \in R$ ?

The idea of the following reduction is as follows: From a Betweenness instance $(V, R)$ we construct an WeAK $D I^{S}$-WO-Orderability instance $(X, \mathcal{X})$. The set $X$ is constructed by adding some auxiliary variables to $V$. Then we use these auxiliary variables to build for every triple $(a, b, c)$ in $R$ a collection of sets that are not $\leq-D I^{S}$-orderable for any linear order on $X$ that violates the betweenness condition for $a, b, c$, i.e., if $b<a, c$ or $a, c<b$ holds. The union of these collections for every triple in $R$ will be the family $\mathcal{X}$. Then we have to show that $\mathcal{X}$ is $\leq-D I^{S}$-orderable if $a<b<c$ or $a>b>c$ holds for all triple $(a, b, c)$ in $R$.

Theorem 46. WEAK $D I^{S}$-WO-ORDERABILITY is NP-complete.

Proof. It is clear that WeAK $D I^{S}$-WO-ORDERABILITy is in NP because we can guess a linear order $\leq$ on $X$ and a linear order $\preceq$ on $\mathcal{X}$ at the same time and then check in polynomial time if $\preceq$ satisfies dominance and strict independence with respect to $\leq$. 
It remains to show that it is also NP-hard. We do this by a reduction from BETWEeNNESS. For the reduction we need two gadgets. First, we need a gadget that guarantees for three elements $a, b, c$ that either $a<b, c$ or $a>b, c$ has to hold. This can be done using a similar idea as for the impossibility result of Barberà and Pattanaik (1984). The second gadget, for elements $x, x^{\prime}, y, y^{\prime}$, leads to a contradiction if $x, x^{\prime}<y, y^{\prime}$ or $y, y^{\prime}<x, x^{\prime}$ holds.

The gadget $A(x, y, z)$ : Let $X=\{x, y, z\}$. Then, we write $A(x, y, z):=\mathcal{P}(X) \backslash\{x\}$. We claim that $A(x, y, z)$ is not $D I^{S}$-orderable with respect to $\leq$ for $y<x<z$ or $z<x<y$. We assume $y<x<z$. The other case can be treated analogously. Assume for the sake of contradiction that there is an order $\preceq$ on $A(x, y, z)$ that satisfies dominance and strict independence. Then, $\{y\} \prec\{y, x\}$ by dominance and hence $\{y, z\} \prec\{y, x, z\}$ by strict independence. On the other hand, $\{x, z\} \prec\{z\}$ by dominance and hence $\{y, x, z\} \prec\{y, z\}$ by strict independence. A contradiction.

The gadget $B\left(x, x^{\prime}, y, y^{\prime}\right)$ : We write $B\left(x, x^{\prime}, y, y^{\prime}\right)$ for the set

$$
\left\{\left\{x, y, y^{\prime}\right\},\left\{x^{\prime}, y, y^{\prime}\right\},\left\{x, x^{\prime}\right\},\left\{x, x^{\prime}, y\right\},\left\{x, x^{\prime}, y^{\prime}\right\},\left\{x, x^{\prime}, y, y^{\prime}\right\},\{y\},\left\{y^{\prime}\right\},\left\{y, y^{\prime}\right\}\right\} .
$$

We claim that $B\left(x, x^{\prime}, y, y^{\prime}\right)$ is not $D I^{S}$-orderable with respect to $\leq$ if $x, x^{\prime}<y, y^{\prime}$ or $y, y^{\prime}<x, x^{\prime}$. We assume $x, x^{\prime}<y<y^{\prime}$. The other cases follow by symmetry. Then, $\left\{x, x^{\prime}\right\} \prec\left\{x, x^{\prime}, y\right\}$ by dominance and hence $\left\{x, x^{\prime}, y^{\prime}\right\} \prec\left\{x, x^{\prime}, y, y^{\prime}\right\}$ by strict independence. On the other hand $\left\{y, y^{\prime}\right\} \prec\left\{y^{\prime}\right\}$ by dominance and hence by strict independence first $\left\{x^{\prime}, y, y^{\prime}\right\} \prec\left\{x^{\prime}, y^{\prime}\right\}$ and second $\left\{x, x^{\prime}, y, y^{\prime}\right\} \prec\left\{x, x^{\prime}, y^{\prime}\right\}$, a contradiction.

The reduction: Given an instance of BeTweEnNESS

$$
\left(V=\left\{v_{1}, \ldots, v_{n}\right\}, T=\left\{\left(v_{i}, v_{j}, v_{k}\right), \ldots,\left(v_{i^{\prime}}, v_{j^{\prime}}, v_{k^{\prime}}\right)\right\}\right)
$$

we build an instance $(X, \mathcal{X})$ of WeAK $D I^{S}$-WO-Orderability. First, we add for every $v_{i} \in V$ an element $v_{i}$ to $X$. Furthermore, we add for every triple $\left(v_{i}, v_{j}, v_{k}\right)$ new elements $y_{i j k}, y_{i j k}^{\prime}, z_{i j k}$ and $z_{i j k}^{\prime}$. If no ambiguity arises, we omit the index $i j k$. Finally, for every triple $\left(v_{i}, v_{j}, v_{k}\right)$ we add sets

$$
\begin{gathered}
A\left(v_{i}, y_{i j k}, y_{i j k}^{\prime}\right), A\left(v_{j}, y_{i j k}, y_{i j k}^{\prime}\right), A\left(v_{i}, z_{i j k}, z_{i j k}^{\prime}\right), A\left(v_{j}, z_{i j k}, z_{i j k}^{\prime}\right), \\
A\left(v_{k}, z_{i j k}, z_{i j k}^{\prime}\right), A\left(v_{i}, y_{i j k}, z_{i j k}\right), A\left(v_{k}, y_{i j k}, z_{i j k}\right), B\left(v_{i}, v_{j}, y_{i j k}, y_{i j k}^{\prime}\right), \\
B\left(v_{i}, v_{k}, z_{i j k}, z_{i j k}^{\prime}\right) \text { and } B\left(v_{j}, v_{k}, z_{i j k}, z_{i j k}^{\prime}\right)
\end{gathered}
$$

to $\mathcal{X}$. We claim that $\mathcal{X}$ is $D I^{S}$-orderable with respect to $\leq$ if and only if the projection of $\leq$ to $V$ is a positive solution to the given Betweenness instance. The idea is, roughly, that none of these gadgets leads to a contradiction if we set either

$$
v_{i}<y<y^{\prime}<v_{j}<z<z^{\prime}<v_{k}
$$

or

$$
v_{i}>y>y^{\prime}>v_{j}>z>z^{\prime}>v_{k},
$$

hence there is a way to avoid a contradiction if either $v_{i}\left\langle v_{j}\left\langle v_{k}\right.\right.$ or $v_{i}>v_{j}>v_{k}$ holds. On the other hand, we can show that there is no way to extend an order that sets either $v_{j}<v_{i}, v_{k}$ or $v_{i}, v_{k}<v_{j}$ without running into a contradiction. 
First, we show that $\mathcal{X}$ is not $D I^{S}$-orderable with respect to $\leq$ if the projection of $\leq$ is not a positive instance of BeTweEnness. Let $\left(v_{i}, v_{j}, v_{k}\right)$ be a triple that is violated by $\leq$, i.e., either $v_{j}<v_{i}, v_{k}$ or $v_{i}, v_{k}<v_{j}$. We assume $v_{j}<v_{i}, v_{k}$. The other case can be treated analogously. Assume for the sake of contradiction that $\mathcal{X}$ is $D I^{S}$-orderable with respect to $\leq$. Then observe that $A\left(v_{i}, y, y^{\prime}\right)$ and $A\left(v_{j}, y, y^{\prime}\right)$ imply that $v_{i}$ and $v_{j}$ cannot lie between $y$ and $y^{\prime}$ in $\leq$. Therefore, we must have either $v_{i}, v_{j}<y, y^{\prime}, y, y^{\prime}<v_{i}, v_{j}, v_{i},<y, y^{\prime}<v_{j}$ or $v_{j},<y, y^{\prime}<v_{i}$. However, the first two cases are ruled out by $B\left(v_{i}, v_{j}, y, y^{\prime}\right)$ and the third case is ruled out by $v_{j}<v_{i}$, hence we know $v_{j}<y, y^{\prime}<v_{i}$. Similarly, $A\left(v_{j}, z, z^{\prime}\right), A\left(v_{k}, z, z^{\prime}\right)$ and $B\left(v_{j}, v_{k}, z, z^{\prime}\right)$ imply $v_{j}<z, z^{\prime}<v_{k}$. By $A\left(v_{i}, y, z\right)$ and $A\left(v_{k}, y, z\right)$ we know that $v_{i}$ and $v_{k}$ cannot lie between $y$ and $z$, hence we must have $v_{j}<y, z<v_{i}, v_{k}$. Now, $A\left(v_{j}, z, z^{\prime}\right), A\left(v_{k}, z, z^{\prime}\right)$ imply that neither $v_{i}$ nor $v_{k}$ can lie between $z$ and $z^{\prime}$. Hence we must have $v_{j}<y, z, z^{\prime}<v_{i}, v_{k}$. However, this is ruled out by $B\left(v_{i}, v_{k}, z, z^{\prime}\right)$. A contradiction.

Now, assume $\leq$ is a positive instance of BetweEnNESs. We extend $\leq$ to an order on $\mathcal{X}$ by setting for all triples $\left(v_{i}, v_{j}, v_{k}\right)$ the order $v_{i}<y<y^{\prime}<v_{j}<z<z^{\prime}<v_{k}$ if $v_{i}<v_{j}<v_{k}$ and $v_{i}>y>y^{\prime}>v_{j}>z>z^{\prime}>v_{k}$ otherwise. We can do this in a way such that there is no element between $y$ and $y^{\prime}$ and $z$ and $z^{\prime}$. Now, we can order the sets made up by the new elements $y, y^{\prime}, z, z^{\prime}$ with an order $\preceq$ satisfying dominance and strict independence: We lift $\leq$ to the singletons and place the sets of the form $\left\{y, y^{\prime}\right\}$ between $\{y\}$ and $\left\{y^{\prime}\right\}$ and sets of the form $\left\{z, z^{\prime}\right\}$ between $\{z\}$ and $\left\{z^{\prime}\right\}$. Finally we place sets of the form $\left\{y^{\prime}, z\right\}$ right after $\left\{y^{\prime}\right\}$.

For every triple $\left(v_{i}, v_{j}, v_{k}\right)$ with auxiliary elements $y, y^{\prime}, z, z^{\prime}$ such that $v_{i}<v_{j}<v_{k}$ holds, we add the sets

$$
\left\{v_{i}, y\right\},\left\{v_{i}, y, y^{\prime}\right\},\left\{v_{i}, y^{\prime}\right\},\left\{v_{i}, y, v_{j}\right\},\left\{v_{i}, y, y^{\prime}, v_{j}\right\},\left\{v_{i}, v_{j}\right\},\left\{v_{i}, y^{\prime}, v_{j}\right\}
$$

in this order just below $\{y\}$. For every of these sets $A$, we have $\min (A)=v_{i}$. Furthermore, for all $A \prec B$ we have $\max (A) \leq \max (B)$. Hence, this sequence satisfies dominance. Next we add

$$
\left\{y, v_{j}\right\},\left\{y, y^{\prime}, v_{j}\right\},\left\{y^{\prime}, v_{j}\right\},\left\{v_{i}, y^{\prime}, z\right\},\left\{y^{\prime}, z\right\},\left\{y^{\prime}, z, v_{k}\right\}
$$

in this order above $\left\{y^{\prime}\right\}$. It can be checked that this also satisfies dominance. Furthermore, we add

$$
\begin{array}{r}
\left\{v_{i}, z\right\},\left\{v_{i}, z, z^{\prime}\right\},\left\{v_{i}, z^{\prime}\right\},\left\{v_{i}, z, v_{k}\right\},\left\{v_{i}, z, z^{\prime}, v_{k}\right\} \\
\left\{v_{i}, v_{k}\right\},\left\{v_{i}, z^{\prime}, v_{k}\right\},\left\{v_{j}, z\right\},\left\{v_{j}, z, z^{\prime}\right\},\left\{v_{j}, z^{\prime}\right\} \\
\left\{v_{j}, z, v_{k}\right\},\left\{v_{j}, z, z^{\prime}, v_{k}\right\},\left\{v_{j}, v_{k}\right\},\left\{v_{j}, z^{\prime}, v_{k}\right\}
\end{array}
$$

in this order just below $\{z\}$. For the first half, we have again $\min (A)=v_{i}$ and $A \prec B$ implies $\max (A) \leq \max (B)$. For the second half, the same holds with $\min (A)=v_{j}$. Therefore, this block satisfies dominance. Furthermore, we have $\max (A) \geq z$ and in the earlier blocks, the only set $B$ with $\max (B)>z$ is $\left\{y^{\prime}, z, v_{k}\right\}$, for which dominance does not imply any preferences with sets in this block. Hence, dominance is also satisfied with in relation to the earlier blocks. Finally, we have $\min (A) \leq z<\max (A)$ for all sets in the block. Therefore, we can place $\{z\}$ above the block without violating dominance. We conclude the construction by placing the sets

$$
\left\{z, v_{k}\right\},\left\{z, z^{\prime}, v_{k}\right\},\left\{z^{\prime}, v_{k}\right\}
$$


in this order above $\left\{z^{\prime}\right\}$. Again, this does not contradict dominance. If $v_{i}>v_{j}>v_{k}$ holds, we produce exactly the reverse order. In order to see that it satisfies strict independence, we have to distinguish two cases: Let $A$ and $B$ be sets in $\mathcal{X}$ such that $A \cup\{x\}$ and $B \cup\{x\}$ are also in $\mathcal{X}$ and $A \prec B$. First, assume $x=y_{i j k}$ for some triple $\left(v_{i}, v_{j}, v_{k}\right)$. First, assume $A \cap\left\{v_{i}, v_{j}, v_{k}\right\}=B \cap\left\{v_{i}, v_{j}, v_{k}\right\}$. Then, we must have $A=\left\{v_{i}, v_{j}\right\}$ and $B=\left\{v_{i}, y^{\prime}, v_{j}\right\}$ and hence $A \cup\{x\} \prec B \cup\{x\}$ by definition. Now, assume $A \cap\left\{v_{i}, v_{j}, v_{k}\right\} \neq B \cap\left\{v_{i}, v_{j}, v_{k}\right\}$. Then, the order of $A$ and $B$ as well as the order of $A \cup\{x\}$ and $B \cup\{x\}$ does not depend on the auxiliary variables. Hence $A \prec B$ implies $A \cup\{x\} \prec B \cup\{x\}$. The cases $x \in\left\{y^{\prime}, x, x^{\prime}\right\}$ are similar.

So assume $x=v_{i}$. Then, observe that if $A \prec B$, because $B$ contains $v_{j}$ or $v_{k}$ and $A$ doesn't, then $A \cup\{x\} \prec B \cup\{x\}$ for the same reason. Otherwise observe that the order of $A$ and $B$ can only depend on the auxiliary elements. However, by assumption, these do not change when we add $x$, hence $A \cup\{x\} \prec B \cup\{x\}$.

A close inspection of this proof shows that (1) we did not use the fact that the lifted order needs to be total and (2) that the lifted order satisfies the extension rule. Hence, the NP-hardness carries over to partial weak $D I^{S}$-orderability and if we add the extension rule.

\section{Corollary 47. Weak $D I^{S} E$-WO-Orderability, Weak $D I^{S}$-PO-Orderability} and WEAK $D I^{S} E$-PO-ORDERABILITY are NP-complete.

Proof. The fact that all three problems are in NP follows by the same argument used to show that WEAK $D I^{S}$-WO-OrderABILITy is in NP. Now, we claim that the same reduction used above shows without modification that all three problems are NP-hard. First of all, the argument that the constructed instance $(X, \mathcal{X})$ is not weakly $D I^{S}$-orderable if $(V, R)$ is not a positive instance of BETWEENNESs does not rely on the fact that $\leq$ needs to be total. ${ }^{10}$ Furthermore, when constructing the order witnessing that $(X, \mathcal{X})$ is weakly $D I^{S}$-orderable if $(V, R)$ is a positive instance of Betweenness, "we lift $\leq$ to the singletons". Therefore, this order satisfies the extension rule by definition.

Unfortunately, this reduction cannot easily be adapted to weak orderability with respect to regular independence. Proposition 10 tells us that at least 6 elements are necessary to produce a conflict between dominance and independence, therefore it seems highly unlikely that one can find a gadget in the style of $A(a, b, c)$ that enforces a specific preference between three elements for dominance and independence. Nevertheless, it seems likely that the problem remains NP-complete when strict independence is replaced with regular independence, though a completely new reduction may be needed to show this.

\subsection{Strengthenings of Dominance}

A possible way to overcome the high complexity of recognizing orderable families could be to strengthen the axioms that we consider. If we consider very strong axioms then only very particular families of sets will be orderable with respect to these axioms. Strict independence is already a very strong axiom, therefore, we focus on strengthenings of dominance.

10. Observe that previous reductions also did not explicitly mention the totality of the lifted order, but used the fact that (strict) independence implies reverse independence, which only holds for total orders. 
Furthermore, we will only study the simplest decision problem, namely $\leq$-LO-orderability. More concretely, we will show that no reasonable strengthening of dominance together with strict independence makes the $\leq$-LO-orderability problem easier. Technically, we say an axiom is dominance-like if it extends dominance and is implied by a very strong axiom that we call maximal dominance, which was first introduced by Maly and Woltran (2017). We show that the $\leq$-LO-orderability problem with respect to strict independence and any dominance-like axiom is NP-complete. First we introduce maximal dominance.

Axiom 48 (Maximal Dominance). For all $A, B \in \mathcal{X}$,

$$
\begin{aligned}
& (\max (A) \leq \max (B) \wedge \min (A)<\min (B)) \text { implies } A \prec B ; \\
& (\max (A)<\max (B) \wedge \min (A) \leq \min (B)) \text { implies } A \prec B .
\end{aligned}
$$

Now, we can define dominance-like axioms. As the name suggests, these are axioms that lie between dominance and maximal dominance.

Definition 49. We say a axiom is dominance-like if it implies dominance and is implied by maximal dominance.

One example of a dominance-like axiom is set-dominance.

Axiom 50 (Set-Dominance). For all $A, X \in \mathcal{X}$ such that $A \cup X \in \mathcal{X}$ and $A \cap X=\emptyset$ :

$$
\begin{aligned}
& y<x \text { for all } x \in X, y \in A \text { implies } A \prec A \cup X ; \\
& x<y \text { for all } x \in X, y \in A \text { implies } A \cup X \prec A .
\end{aligned}
$$

Furthermore, if we consider Fishburn's and Gärdenfors' extensions as axioms, they are both dominance-like.

The following reduction is based on a similar idea as the one used by Maly and Woltran (2017) but is significantly more general and hence proves a much stronger result. We prove the NP-hardness of $\leq$-orderability by a reduction from BetweEnNEss, as in the case of weak orderability, However, this time the BeTweEnNess instance is not encoded in the linear order on $X$ but in the linear order on $\mathcal{X}$.

Theorem 51. Let $A$ be a dominance-like axiom. Then it is NP-hard to decide for a given triple $(X, \mathcal{X}, \leq)$ if there exists a linear order on $\mathcal{X}$ that satisfies axiom $A$ and strict independence.

Proof. Let $(V, R)$ be an instance of Betweenness with $V=\left\{v_{1}, v_{2}, \ldots, v_{n}\right\}$. We construct a triple $(X, \mathcal{X},<)$ such that

- if $(V, R)$ is a positive instance of Betweenness, then there is a linear order $\preceq$ on $\mathcal{X}$ that satisfies maximal dominance and strict independence w.r.t. $\leq$,

- if $(V, R)$ is a negative instance of BeTweEnNEss, then there exists no linear order $\preceq$ on $\mathcal{X}$ that satisfies dominance and strict independence w.r.t. $\leq$. 


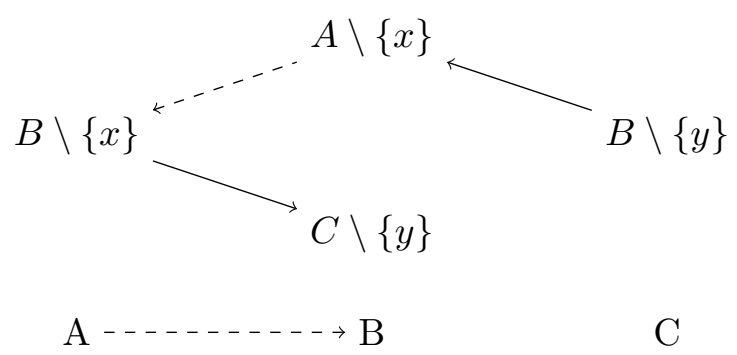

Figure 5: Family that forces that $A \prec B$ leads to $B \prec C$

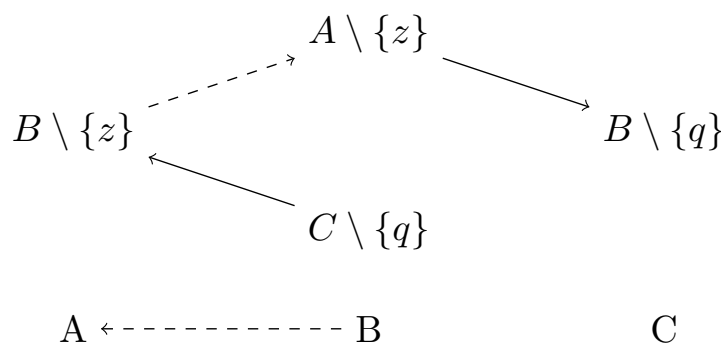

Figure 6: Family that forces that $A \succ B$ leads to $B \succ C$

Then, for any dominance-like axiom $A$ we know that there exists a linear order on $\mathcal{X}$ that satisfies axiom $A$ and strict independence w.r.t. $\leq$ if and only if $(V, R)$ is a positive instance of BetweEnness.

We set $X=\{1,2, \ldots, N\}$ equipped with the usual linear order, for $N$ large enough. We will clarify later what large enough means. Then, we construct the family $\mathcal{X}$ stepwise. The family contains for every $v_{i} \in V$ a set $V_{i}$ of the following form:

$$
V_{i}:=\{K+i, \ldots, N-(K+i)\}
$$

Here, $K$ is a large enough constant. Again we will clarify later what large enough means.

Furthermore, for every triple from $R$ we want to enforce $A \prec B \prec C$ or $A \succ B \succ C$ by adding two families of sets as shown in Figure 5 and Figure 6 with $q, x, y, z \in X$. The solid arrows represent preferences that are forced through dominance and strict independence. The family in Figure 5 makes sure that every total strict order satisfying independence that contains $A \prec B$ must also contain $B \prec C$. Similarly, the family in Figure 6 makes sure that $A \succ B$ leads to $B \succ C$.

We implement this idea for all triples inductively. For every $1 \leq i \leq|R|$, pick a triple $\left(v_{l}, v_{j}, v_{m}\right) \in R$ and set $k=K+n+4 i$. Let $(A, B, C)=\left(V_{l}, V_{j}, V_{m}\right)$ be the triple of sets encoding the triple of elements $\left(v_{l}, v_{j}, v_{m}\right)$. We add the following sets:

$$
\begin{array}{r}
A \backslash\{k\}, B \backslash\{k\}, B \backslash\{k+1\}, C \backslash\{k+1\}, \\
\quad A \backslash\{k+2\}, B \backslash\{k+2\}, B \backslash\{k+3\}, C \backslash\{k+3\} .
\end{array}
$$




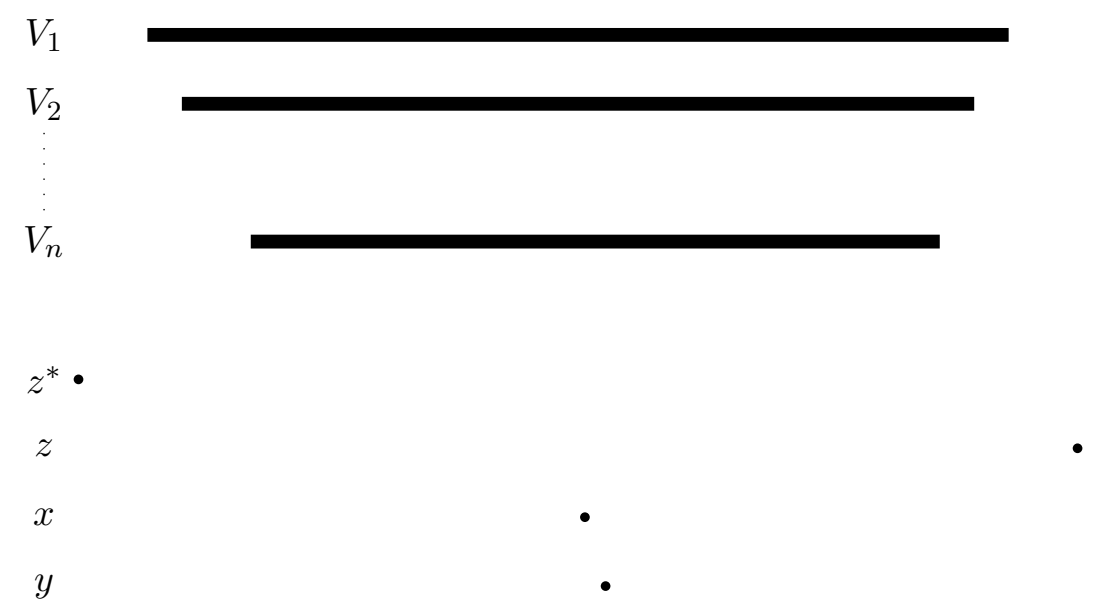

Figure 7: Sketch of the sets $V_{1}, \ldots, V_{n}, x, y, z$ and $z^{*}$.

We call the sets encoding the elements of $V$ together with the sets added in this step the Class 1 sets. These sets correspond to the sets $A \backslash\{x\}, B \backslash\{x\}, \ldots, C \backslash\{q\}$ in Figure 5 and Figure 6. Observe that the inductive construction guarantees that every constructed set is unique. We now have to force the preferences

$$
\begin{aligned}
A \backslash\{k\} \prec B \backslash\{k+1\}, C \backslash\{k+1\} \prec B \backslash\{k\}, \\
B \backslash\{k+3\} \prec A \backslash\{k+2\}, B \backslash\{k+2\} \prec C \backslash\{k+3\} .
\end{aligned}
$$

We define for every pair $A, B \in \mathcal{X}$ a family of sets $\mathcal{S}(A, B)$ forcing $A \prec B$. Assume $\min (B) \leq \min (A)$ and $\max (A) \leq \max (B)$. Then, $\mathcal{S}(A, B)$ contains the following sets

$$
\left\{x_{A B}\right\},\left\{x_{A B}, y_{A B}\right\},\left\{y_{A B}\right\},\left\{x_{A B}, z_{A B}\right\},\left\{y_{A B}, z_{A B}^{*}\right\}, A \cup\left\{z_{A B}\right\}, B \cup\left\{z_{A B}^{*}\right\}
$$

where $z_{A B}^{*}<\min (B)<\min (A)<x_{A B}<y_{A B}<\max (A)<\max (B)<z_{A B}$ holds (See Figure 7).

Additionally, we add sets that enforce $A \cup\left\{z_{A B}\right\} \prec\left\{x_{A B}, z_{A B}\right\}$ by dominance: Let $A=\left\{a_{1}, \ldots, a_{l}\right\}$ be enumerations of $A$ such that $i<j$ implies $a_{i}<a_{j}$. We add

$$
\left\{z_{A B}\right\},\left\{a_{l}, z_{A B}\right\},\left\{a_{l-1}, a_{l}, z_{A B}\right\}, \ldots,\left\{a_{2}, \ldots, z_{A B}\right\} \text { and }\left\{a_{1}, z_{A B}\right\}
$$

to $\mathcal{X}$. This forces $\left\{a_{2}, \ldots, z_{A B}\right\} \prec\left\{z_{A B}\right\}$ by dominance and hence by one application of strict independence $A \cup\left\{z_{A B}\right\} \prec\left\{a_{1}, z_{A B}\right\}$. Finally, we add the sets $\left\{a_{1}\right\},\left\{a_{1}, x_{A B}\right\}$ and $\left\{a_{1}, x_{A B}, z_{A B}\right\}$, which leads to $\left\{a_{1}, z_{A B}\right\} \prec\left\{a_{1}, x_{A B}, z_{A B}\right\}$. Then we have

$$
A \cup\left\{z_{A B}\right\} \prec\left\{a_{1}, z_{A B}\right\} \prec\left\{a_{1}, x_{A B}, z_{A B}\right\} \prec\left\{x_{A B}, z_{A B}\right\}
$$

hence $A \cup\left\{z_{A B}\right\} \prec\left\{x_{A B}, z_{A B}\right\}$. Therefore, we have $A \preceq\left\{x_{A B}\right\}$ by reverse independence. Analogously, we enforce $\left\{y_{A B}\right\} \preceq B$. Therefore, transitivity implies $A \prec B$ by $A \preceq\left\{x_{A B}\right\} \prec$ $\left\{y_{A B}\right\} \preceq B$. The case $\min (A) \leq \min (B)$ and $\max (B) \leq \max (A)$ can be treated analogously. 
Now, we add the following families of sets to enforce the desired preferences

$$
\begin{aligned}
\mathcal{S}(A \backslash\{k\}, B \backslash\{k+1\}), \mathcal{S}(C \backslash\{k+1\}, B \backslash\{k\}), & \\
& \mathcal{S}(B \backslash\{k+3\}, A \backslash\{k+2\}), \mathcal{S}(B \backslash\{k+2\}, C \backslash\{k+3\}) .
\end{aligned}
$$

We call the sets added in this step the Class 2 sets.

We repeat this with a new triple $\left(v_{i}^{\prime}, v_{j}^{\prime}, v_{m}^{\prime}\right) \in R$ until we treated all triples in $R$. By this construction every linear order on $\mathcal{X}$ that satisfies dominance and strict independence must set the preferences listed in $(\star)$. This concludes the construction of $\mathcal{X}$. We now can determine the necessary sizes for $N$ and $K . N$ and $K$ need to be large enough such that all $z_{A B}^{*}$ used in the construction are smaller than $K$, all $z_{A B}$ are larger than $N-K$ and all $x_{A B}$ and $y_{A B}$ are larger than $K+n+4|R|$. It is clear that this can be achieved with $N$ and $K$ that are polynomial in $|(V, R)|$.

Now, assume there is a linear order on $\mathcal{X}$ satisfying dominance and strict independence. We claim that the relation defined by $v_{i} \leq v_{j}$ iff $V_{i} \preceq V_{j}$ is a positive witness for $(V, R)$. By definition this is a linear order. So assume there is a triple $(a, b, c)$ such that $a>b<c$ or $a<b>c$ holds. We treat the first case in detail: $a>b<c$ implies $A \succ B \prec C$. This implies by the strictness of $\prec$ and strict dominance $A \backslash\{k\} \succ B \backslash\{k\}$ and $B \backslash\{k+1\} \prec C \backslash\{k+1\}$. However, then

$$
A \backslash\{k\} \succ B \backslash\{k\} \succ C \backslash\{k+1\} \succ B \backslash\{k+1\} \succ A \backslash\{k\}
$$

contradicts the assumption that $\prec$ is transitive and irreflexive. Similarly, the second case leads to a contradiction. This shows that if $(V, R)$ is a negative instance of BeTweEnness, then there is no order on $\mathcal{X}$ that satisfies dominance and strict independence with respect to $\leq$.

Now, assume that there is a linear order on $V$ satisfying the restrictions from $R$. We use this to construct a linear order on $\mathcal{X}$ that satisfies maximal dominance and strict independence with respect to $\leq$. First, we add all preferences implied by maximal dominance. Observe that no two Class 1 sets are comparable by maximal dominance. Moreover, we set $V_{i} \preceq V_{j}$ iff $v_{i} \leq v_{j}$ holds. Then, we project this order to all sets of the form $V_{i} \backslash\{x\}$, We claim that this order satisfies all applications of strict independence between Class 1 sets. If $A=V_{i}$ for $i \leq n$, then there is no set $A \cup\{x\}$ in $\mathcal{X}$. If $A=V_{i} \backslash\{x\}$ for some $i \leq n$ and $x \in X$, then $x$ is the only element of $X$ such that $A \cup\{x\} \in \mathcal{X}$ holds. But then there can only be one other set $B$ with $B \cup\{x\} \in \mathcal{X}$ and $B=V_{j} \backslash\{x\}$ hence a preference between $A$ and $B$ was introduced by reverse strict independence.

Next, we consider the Class 2 sets. We distinguish three types of Class 2 sets. We say a set $X$ is

- type 1 if $z_{A B}^{*} \in X$,

- type 2 if $z_{A B}, z_{A B}^{*} \notin X$,

- type 3 if $z_{A B} \in X$.

Then, we set $X \prec Y$ if type $(X)<\operatorname{type}(Y)$. Furthermore, for all type 1 sets $X, Y$ we set $X \prec Y$ if 
- $z_{A B}^{*} \in X, z_{C D}^{*} \in Y$ and $z_{A B}^{*}<z_{C D}^{*}$,

- $z_{A B}^{*} \in X, Y$ and $x_{A B} \notin X, x_{A B} \in Y$,

- $z_{A B}^{*}, x_{A B} \in X, Y$ and $\max (X \triangle Y) \in Y$,

- $z_{A B}^{*} \in X, Y x_{A B} \notin X, Y$ and $\max (X \triangle Y) \in Y$.

It is straightforward to check that this order satisfies strict independence and is compatible with maximal dominance on all type 1 sets. Similarly, we set for all type 3 sets

- $z_{A B} \in X, z_{C D} \in Y$ and $z_{A B}<z_{C D}$,

- $z_{A B} \in X, Y$ and $x_{A B} \notin X, x_{A B} \in Y$,

- $z_{A B}, x_{A B} \in X, Y$ and $\min (X \triangle Y) \in X$,

- $z_{A B} \in X, Y x_{A B} \notin X, Y$ and $\min (X \triangle Y) \in X$.

Again, it is straightforward to check that this order satisfies strict independence and is compatible with maximal dominance on all type 3 sets.

Now, we covered all possible applications of strict independence on Class 1 sets and on type 1 and 3 sets. The only possible application of strict independence that includes Class 1 and Class 2 sets is adding $z_{A B}$ or $z_{A B}^{*}$ to a Class 1 set and a Class 2 set. Then, by construction both resulting sets are type 1 resp. 3 sets. Therefore, we can apply reverse strict independence. By construction, this does not lead to a cycle if any only if we started with an positive instance of BETWEENNESS.

It remains to consider applications of strict independence that include type 2 sets. All type 2 sets are of one of the following forms:

$$
\left\{x_{A B}\right\},\left\{y_{A B}\right\},\left\{x_{A B}, y_{A B}\right\},\left\{a_{1}\right\},\left\{a_{1}, x_{A B}\right\},\left\{b_{l}\right\},\left\{b_{l}, y_{A B}\right\}
$$

Now, any application of strict independence where the same element is added to two singletons is clearly satisfied by any order that satisfies maximal dominance. This leaves the case that $z_{A B}$ or $z_{A B}^{*}$ is added to two different type 2 sets. Now, by construction, we have $\left\{a_{1}\right\} \prec\left\{a_{1}, x_{A B}\right\} \prec\left\{x_{A B}\right\}$ and $\left\{a_{1}, z_{A B}\right\} \prec\left\{a_{1}, x_{A B}, z_{A B}\right\} \prec\left\{x_{A B}, z_{A B}\right\}$. Therefore, the case where $z_{A B}$ is added is satisfied. The case that $z_{A B}^{*}$ is added is similar.

It can be checked that this covers all possible applications of strict independence. Finally, we can extend this order to a weak order because extensions do not produce new instances of strict independence.

\section{Discussion}

Lifting a preference order on elements of some universe to a preference order on subsets of this universe respecting certain axioms is a fundamental problem, but impossibility results by Kannai and Peleg and by Barberà and Pattanaik pose severe limits on when such liftings exist if all non-empty subsets of the universe have to be ordered. We observed that these impossibility results may be avoided if not all non-empty subsets of the universe have to be 
ordered. This raises the questions how hard it is to recognize families for which dominance and (strict) independence are jointly satisfiable.

Our results show that, in general, we cannot easily recognize families of sets on which we can avoid Kannai and Peleg's or Barbera and Pattanaik's impossibility results. While these results are largely negative, we observe that determining if the family of sets is strongly orderable is important in many applications but not always time-sensitive. Therefore, we believe that the order lifting approach studied here may be useful for applications where partial orders are acceptable, as we can construct a partial order that satisfies dominance and strict independence in polynomial time, whenever such an order exists. Moreover, we have seen that we can always find a partial order that satisfies dominance and weak independence. This provides a practical solution if partial orders are acceptable and the considered family of sets turns out not to be strongly $D I^{S}(E)$-orderable or if the high complexity means that checking for strong orderability with respect to dominance and strict independence is not feasible.

Additionally, we observe that an important implication of the presented hardness results is that the characterization results from Maly et al. (2019) can not easily be generalized to arbitrary families of sets. Indeed, any property that characterizes strong orderability on arbitrary families of sets must be $\Pi_{2}^{p}$-complete to check. This precludes nearly all simple properties of families of sets from characterizing strong orderability. On the other hand, we observe that hypergraph colorability is NP-hard to check. Hence, a generalization of the characterization of weak orderability in terms of colorability by Maly et al. (2019) is not necessarily ruled out by the NP-completeness of checking weak $D I^{S}$-orderability.

There are some remaining gaps in our results that are left to future work. First of all, the complexity of weak $D I$ - and $D I E$-orderability is left open. Furthermore, it remains open if strengthening strict independence influences the complexity of the studied problems. Moreover, our research opens several new directions for future studies.

First of all, Kannai and Peleg's or Barbera and Pattanaik's impossibility results are the most prominent but certainly not the only impossibility results. Other interesting impossibility results where for example proven by Geist and Endriss (2011) and Jones and Sugden (1982). These other results also assume that the whole power set needs to be ordered. Therefore, one could study the questions raised in this thesis the same way also for these other impossibility results.

Moreover, the representation of families by boolean circuits is extremely powerful and therefore leads to an exponential blow up in complexity. On the other hand, the representation of sets by the connectivity condition on graphs used by Maly et al. (2019) can decrease the complexity of the studied problems, but is very restrictive. Therefore, future research is needed to identify succinct representations that are as expressive as possible without an exponential blow up in complexity. Implicit representations of families of sets that appear in important applications would be especially interesting to study. Knowledge representation often uses logic formalisms towards this end. For instance, formulas can be viewed as concise representations of the families of their models. Together with an order of the atoms in the formulas, it is natural to ask how to rank these models and for which classes of formulas such a lifting respects certain criteria.

We note that the presented reductions all require relatively large sets of objects $X$. However, in many practical applications the number of objects is often rather small. For 
example, in many voting scenarios the number of candidates seldomly exceeds 10 . Therefore, it would be worth exploring the parametrized complexity of the discussed problems in particular with regards to the parameter $|X|$.

Furthermore, while we studied the effect of allowing the lifted order to be incomplete, we always assumed that the order on $X$ is a linear order. However, there are important applications, for example in argumentation (Beirlaen et al., 2018; Maly \& Wallner, 2021), where it is necessary to lift partial orders. Hence, it would be interesting to study the effect of allowing the order on $X$ to be incomplete and, in particular, to explore whether the studied problems become easier if only few comparisons between objects are specified. This could either be achieved by means parametrized complexity theory or by aiming for classification results in the style of Maly et al. (2019).

Finally, several interesting questions arise when applying the order lifting approach in specific settings. For example, if lifted orders are used in voting or (ordinal) allocation to generate the input to a voting rule or allocation method, then any axiom essentially represents a domain restriction in that it forces a specific structure on the lifted orders. For example, if the lifted order is a linear order that satisfies strict independence and we have four alternatives $A, B, C, D$ such that $C=A \cup\{x\}$ and $D=B \cup\{x\}$, then the lifted orders of all voters must order $A$ and $B$ the same as $C$ and $D$. This added structure might influence which voting rule or allocation method satisfies the most desirable axioms. Therefore, it would be highly interesting to study the interplay between lifting procedures and axioms on the one hand and voting rules and other social choice mechanisms on the other hand.

\section{Acknowledgments}

The author wants to thank the anonymous reviewers for their valuable feedback and helpful suggestions. Furthermore, the author would like to thank the Austrian Science Fund (FWF) which supported this work under Grant number P31890 and J4581. This paper is an extension of an AAAI 2020 paper (Maly, 2020a). Moreover, the results presented in this paper also appeared in the PhD thesis of the author (Maly, 2020b).

\section{References}

Allouche, T., Escoffier, B., Moretti, S., \& Öztürk, M. (2020). Social ranking manipulability for the cp-majority, banzhaf and lexicographic excellence solutions. In Bessiere, C. (Ed.), Proceedings of the 29th International Joint Conference on Artificial Intelligence, IJCAI 2020, pp. 17-23. ijcai.org.

Arlegi, R. (2003). A note on Bossert, Pattanaik and Xu's "Choice under complete uncertainty: Axiomatic characterization of some decision rules". Economic Theory, 22(1), $219-225$.

Balcázar, J. L., Lozano, A., \& Torán, J. (1992). The complexity of algorithmic problems on succinct instances. In Computer Science: Research and Applications, pp. 351-377. Springer US.

Bandyopadhyay, T. (1988). Extension of an order on a set to the power set: some further observations. Mathematical Social Sciences, 15(1), $81-85$. 
Barberà, S. (1977). Manipulation of social decision functions. Journal of Economic Theory, 15(2), 266-278.

Barberà, S. (2011). Strategyproof social choice. In Handbook of Social Choice and Welfare, Vol. 2, pp. 731-831. Elsevier.

Barberà, S., Barret, C. R., \& Pattanaik, P. K. (1984). On some axioms for ranking sets of alternatives. Journal of Economic Theory, 33(2), 301-308.

Barberà, S., Bossert, W., \& Pattanaik, P. K. (2004). Ranking sets of objects. In Handbook of Utility Theory, pp. 893-977. Springer.

Barberà, S., \& Pattanaik, P. K. (1984). Extending an order on a set to the power set: some remarks on Kannai and Peleg's approach. Journal of Economic Theory, 32(1), $185-191$.

Beirlaen, M., Heyninck, J., Pardo, P., \& Straßer, C. (2018). Argument strength in formal argumentation. IfCoLog Journal of Logics and their Applications, 5(3), 629-676.

Bernardi, G., Lucchetti, R., \& Moretti, S. (2019). Ranking objects from a preference relation over their subsets. Social Choice and Welfare, 52, 589-606.

Booth, R., Chevaleyre, Y., Lang, J., Mengin, J., \& Sombattheera, C. (2010). Learning conditionally lexicographic preference relations. In Proceedings of ECAI 2010, pp. 269-274. IOS Press.

Bossert, W. (1995). Preference extension rules for ranking sets of alternatives with a fixed cardinality. Theory and Decision, 39(3), 301-317.

Bossert, W., Pattanaik, P. K., \& Xu, Y. (2000). Choice under complete uncertainty: axiomatic characterizations of some decision rules. Economic Theory, 16 (2), 295-312.

Bossert, W., \& Suzumura, K. (2012). Revealed preference and choice under uncertainty. SERIEs: Journal of the Spanish Economic Association, 3(1), 247-258.

Boutilier, C., Brafman, R., Domshlak, C., Hoos, H., \& Poole, D. (2004). CP-nets: A tool for representing and reasoning with conditional ceteris paribus preference statements. Journal of Artificial Intelligence Research, 21, 135-191.

Boutilier, C., \& Rosenschein, J. S. (2016). Incomplete information and communication in voting. In Handbook of Computational Social Choice, pp. 223-257. Cambridge University Press.

Bouveret, S., Chevaleyre, Y., \& Maudet, N. (2016). Fair allocation of indivisible goods. In Brandt, F., Conitzer, V., Endriss, U., Lang, J., \& Procaccia, A. D. (Eds.), Handbook of Computational Social Choice, pp. 284-310. Cambridge University Press.

Bouveret, S., Endriss, U., \& Lang, J. (2009). Conditional importance networks: A graphical language for representing ordinal, monotonic preferences over sets of goods. In Proceedings of the 21th International Joint Conference on Artificial Intelligence, IJCAI 2009, pp. 67-72. AAAI Press.

Brandt, F., Brill, M., \& Harrenstein, P. (2016). Tournament solutions. In Brandt, F., Conitzer, V., Endriss, U., Lang, J., \& Procaccia, A. D. (Eds.), Handbook of Computational Social Choice, pp. 57-84. Cambridge University Press. 
Brandt, F., Saile, C., \& Stricker, C. (2018). Voting with ties: Strong impossibilities via SAT solving. In Proceedings of the 17th International Conference on Autonomous Agents and Multiagent Systems (AAMAS), pp. 1285-1293. IFAAMAS.

Bräuning, M., \& Hüllermeier, E. (2012). Learning conditional lexicographic preference trees. In Preference Learning: Problems and Applications in AI, Proceedings of ECAI 2012 Workshop.

Brewka, G., Benferhat, S., \& Le Berre, D. (2004). Qualitative choice logic. Artif. Intell., $157(1-2), 203-237$.

Brewka, G., Niemelä, I., \& Truszczyński, M. (2003). Answer set optimization. In Proceedings of the Eighteenth International Joint Conference on Artificial Intelligence, IJCAI 2003, pp. 867-872. Morgan Kaufmann.

Can, B., Erdamar, B., \& Sanver, R. (2009). Expected utility consistent extensions of preferences. Theory and Decision, 67(2), 123-144.

Daly, L. W. L. W. (1967). Contributions to a History of Alphabetization in Antiquity and Middle Ages. Bruxelles : Latomus.

Darwiche, A., \& Marquis, P. (2002). A knowledge compilation map. Journal of Artificial Intelligence Research (JAIR), 17(1), 229-264.

Domshlak, C., Hüllermeier, E., Kaci, S., \& Prade, H. (2011). Preferences in AI: An overview. Artificial Intelligence, 175(7-8), 1037 - 1052.

Dubois, D., \& Prade, H. (1991). Possibilistic logic, preferential models, non-monotonicity and related issues. In Proceedings of the 12th International Joint Conference on Artificial Intelligence, IJCAI-1991, pp. 419-425. Morgan Kaufmann.

Duggan, J., \& Schwartz, T. (2000). Strategic manipulability without resoluteness or shared beliefs: Gibbard-Satterthwaite generalized. Social Choice and Welfare, 17(1), 85-93.

Dutta, B., \& Sen, A. (2005). Ranking opportunity sets and arrow impossibility theorems: Correspondence results. Journal of Economic Theory, 71, 90-101.

Faliszewski, P., Skowron, P., Slinko, A., \& Talmon, N. (2017). Multiwinner voting: A new challenge for social choice theory. Trends in Computational Social Choice, 74, 27-47.

Fishburn, P. C. (1972). Even-chance lotteries in social choice theory. Theory and Decision, $3(1), 18-40$.

Fishburn, P. C. (1974). Lexicographic orders, utilities and decision rules: A survey. Management Science, 20(11), 1442-1471.

Fishburn, P. C. (1984). Comment on the Kannai-Peleg impossibility theorem for extending orders. Journal of Economic Theory, 32(1), 176-179.

Galperin, H., \& Wigderson, A. (1983). Succinct representations of graphs. Information and Control, 56(3), $183-198$.

Gärdenfors, P. (1976). Manipulation of social choice functions. Journal of Economic Theory, 13(2), 217-228. 
Geist, C., \& Endriss, U. (2011). Automated search for impossibility theorems in social choice theory: Ranking sets of objects. Journal of Artificial Intelligence Research (JAIR), 40, 143-174.

Gibbard, A. (1973). Manipulation of voting schemes: A general result. Econometrica, 41(4), $587-601$.

Haret, A., Khani, H., Oztürk, S. M., \& Meltem (2018). Ceteris paribus majority for social ranking. In Proceedings of the Twenty-Seventh International Joint Conference on Artificial Intelligence, IJCAI 2018, pp. 303-309. ijcai.org.

Holzman, R. (1984). A note on the redundancy of an axiom in the Pattanaik-Peleg characterization of the lexicographic Maximin extension. Social Choice and Welfare, 1(2), $123-125$.

Jones, P., \& Sugden, R. (1982). Evaluating choice. International Review of Law and Economics, 2(1), 47-65.

Kaci, S. (2011). Working with Preferences: Less Is More. Springer.

Kannai, Y., \& Peleg, B. (1984). A note on the extension of an order on a set to the power set. Journal of Economic Theory, 32(1), 172-175.

Kelly, J. S. (1977). Strategy-proofness and social choice functions without singlevaluedness. Econometrica, 45(2), 439-446.

Khani, H., Moretti, S., \& Oztürk, M. (2019). An ordinal Banzhaf index for social ranking. In Proceedings of the Twenty-Eighth International Joint Conference on Artificial Intelligence, IJCAI 2019, pp. 378-384. IJCAI Organization.

Kilgour, D. M. (2016). Approval elections with a variable number of winners. Theory and Decision, 81, 199-211.

Kim, K. H., \& Roush, F. W. (1980). Preferences on subsets. Journal of Mathematical Psychology, 21(3), $279-282$.

Kraft, C. H., Pratt, J. W., \& Seidenberg, A. (1959). Intuitive probability on finite sets. The Annals of Mathematical Statistics, 30(2), 408-419.

Kranich, L. (1996). Equitable opportunities: An axiomatic approach. Journal of Economic Theory, 71(1), 131 - 147.

Lang, J., \& Xia, L. (2016). Voting in combinatorial domains. In Handbook of Computational Social Choice, pp. 197-221. Cambridge University Press.

Larbi, R. B., Konieczny, S., \& Marquis, P. (2010). A characterization of optimality criteria for decision making under complete ignorance. In Proceedings of the Twelfth International Conference on Principles of Knowledge Representation and Reasoning, KR 2010, pp. 172-181. AAAI Press.

Liu, X., \& Truszczynski, M. (2015). Learning partial lexicographic preference trees over combinatorial domains. In Proceedings of the Twenty-Ninth AAAI Conference on Artificial Intelligence, pp. 1539-1545. AAAI Press.

Lucchetti, R., Moretti, S., \& Patrone, F. (2015). Ranking sets of interacting objects via semivalues. Top, 23(2), 567-590. 
Maly, J. (2020a). Lifting preferences over alternatives to preferences over sets of alternatives: The complexity of recognizing desirable families of sets. In Proceedings of the ThirtyFourth AAAI Conference on Artificial Intelligence, pp. 2152-2159. AAAI Press.

Maly, J. (2020b). Ranking Sets of Objects: How to Deal with Impossibility Results. Ph.D. thesis, TU Wien.

Maly, J., Truszczyński, M., \& Woltran, S. (2018). Preference orders on families of sets when can impossibility results be avoided?. In Proceedings of the Twenty-Seventh International Joint Conference on Artificial Intelligence, IJCAI 2018, pp. 433-439. ijcai.org.

Maly, J., Truszczynski, M., \& Woltran, S. (2019). Preference orders on families of sets when can impossibility results be avoided?. Journal of Artificial Intelligence Research (JAIR), 66, 1147-1197.

Maly, J., \& Wallner, J. P. (2021). Ranking sets of defeasible elements in preferential approaches to structured argumentation: Postulates, relations, and characterizations. In Proceedings of the Thirty-Fifth AAAI Conference on Artificial Intelligence (AAAI 2021). AAAI Press. In press.

Maly, J., \& Woltran, S. (2017). Ranking specific sets of objects. Datenbank-Spektrum, $17(3), 255-265$.

Moretti, S., \& Öztürk, M. (2017). Some axiomatic and algorithmic perspectives on the social ranking problem. In Algorithmic Decision Theory - 5th International Conference, ADT 2017, Proceedings, Vol. 10576 of LNCS, pp. 166-181. Springer.

Moretti, S., \& Tsoukiàs, A. (2012). Ranking sets of possibly interacting objects using Shapley extensions. In Proceedings of the Thirteenth International Conference on Principles of Knowledge Representation and Reasoning, KR 2012, pp. 199-209. AAAI Press.

Murray, C. D., \& Williams, R. R. (2017). On the (non) NP-hardness of computing circuit complexity. Theory of Computing, 13(4), 1-22.

Opatrny, J. (1979). Total ordering problem. SIAM Journal on Computing, 8(1), 111-114.

Papadimitriou, C. H. (1994). Computational Complexity. Addison-Wesley.

Papadimitriou, C. H., \& Yannakakis, M. (1986). A note on succinct representations of graphs. Information and Control, 71(3), 181 - 185.

Pattanaik, P. K., \& Xu, Y. (1990). On ranking opportunity sets in terms of freedom of choice. Recherches Économiques de Louvain/Louvain Economic Review, 56(3-4), 383-390.

Satterthwaite, M. A. (1975). Strategy-proofness and Arrow's conditions: Existence and correspondence theorems for voting procedures and social welfare functions. Journal of Economic Theory, 10(2), 187 - 217.

Terzopoulou, Z., \& Endriss, U. (2019). Aggregating incomplete pairwise preferences by weight. In Proceedings of the Twenty-Eighth International Joint Conference on Artificial Intelligence, IJCAI 2019, pp. 595-601. IJCAI Organization. 
Ranking Sets of Objects: The Complexity of Avoiding Impossibility Results

Veith, H. (1998). Succinct representation, leaf languages, and projection reductions. Information and Computation, 142(2), 207-236.

Xia, L., \& Conitzer, V. (2011). A maximum likelihood approach towards aggregating partial orders. In Proceedings of the Twenty-Second International Joint Conference on Artificial Intelligence, IJCAI 2011, pp. 446-451. AAAI Press. 